
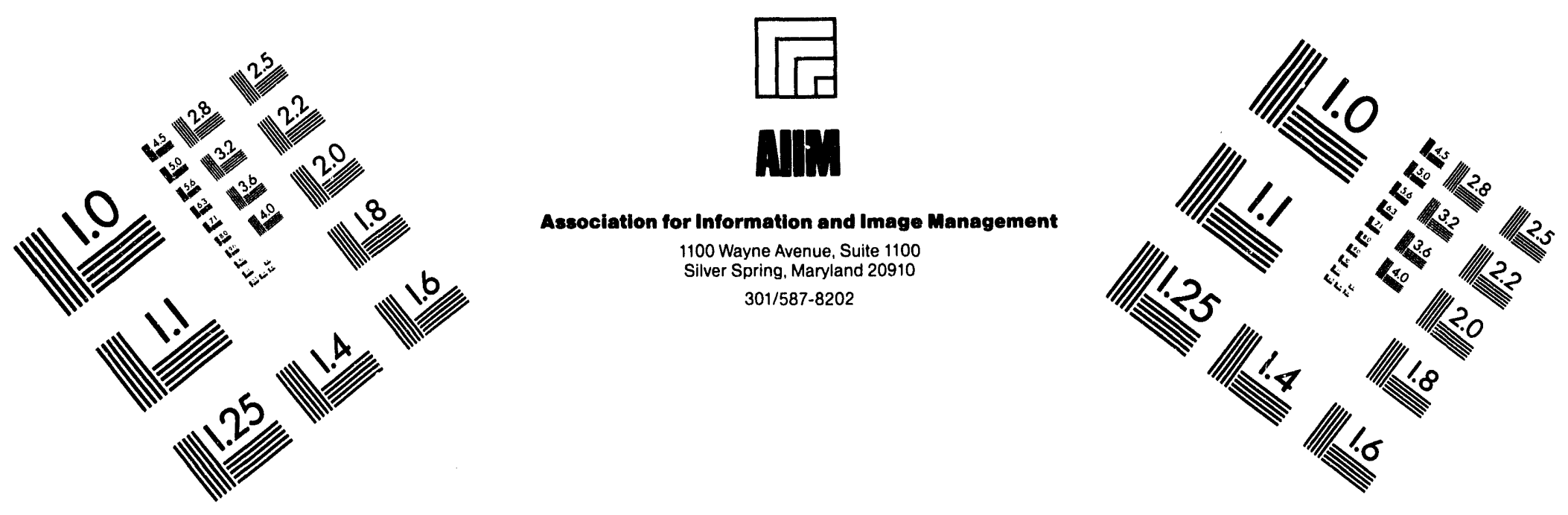

\title{
Centimeter
}

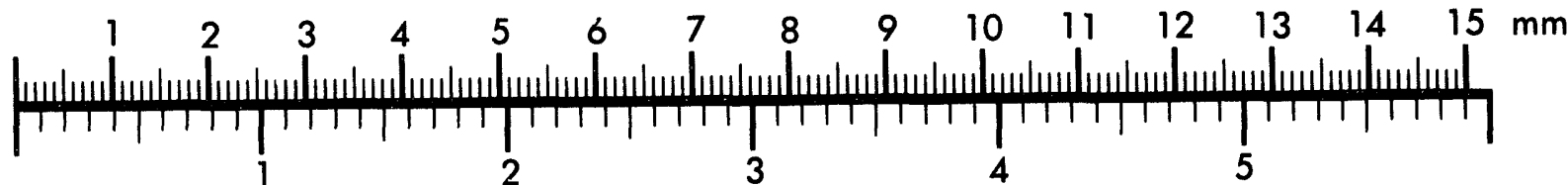
Inches
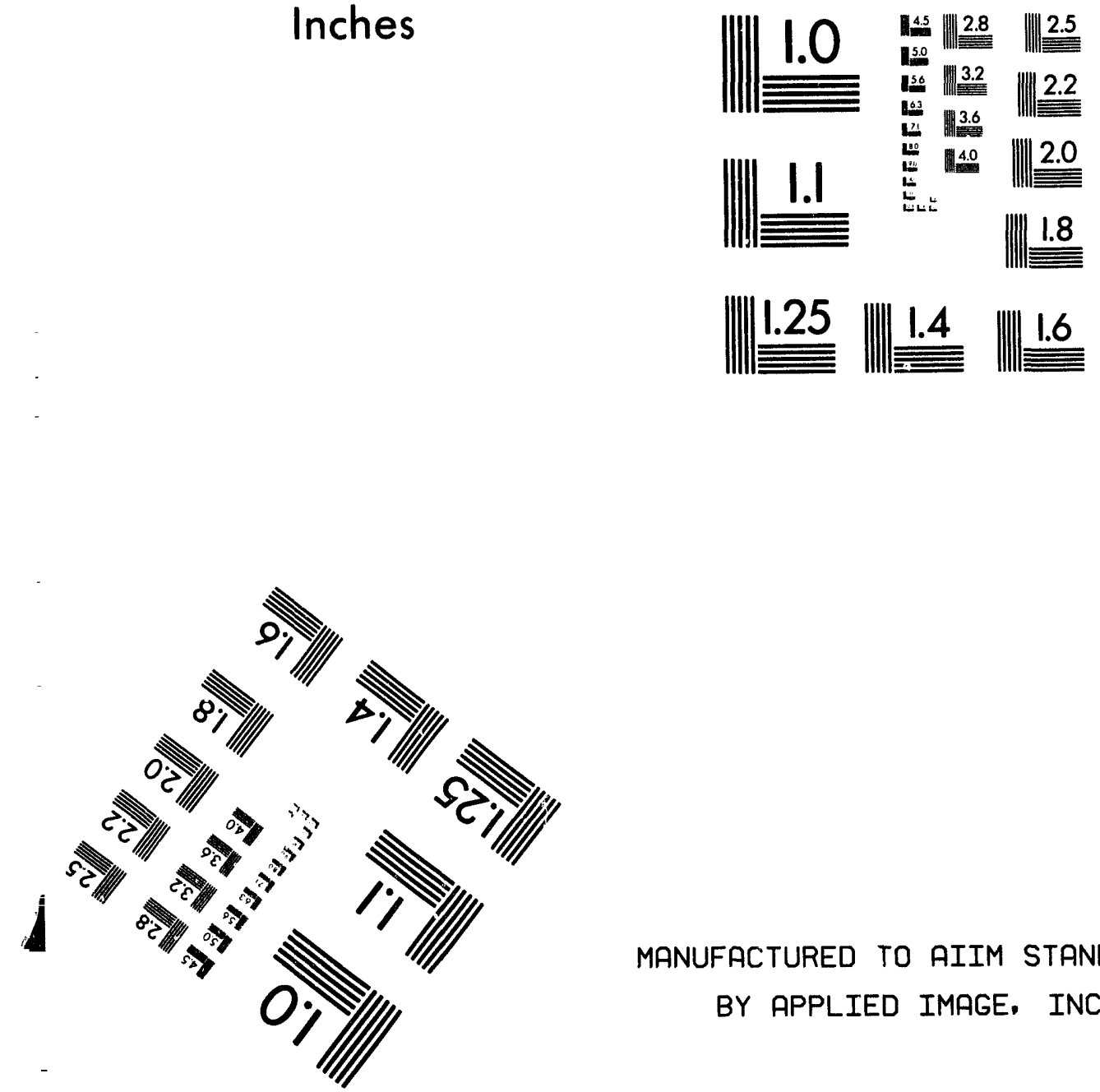

MANUFACTURED TO AIIM STANDARDS

BY APPLIED IMAGE, INC.

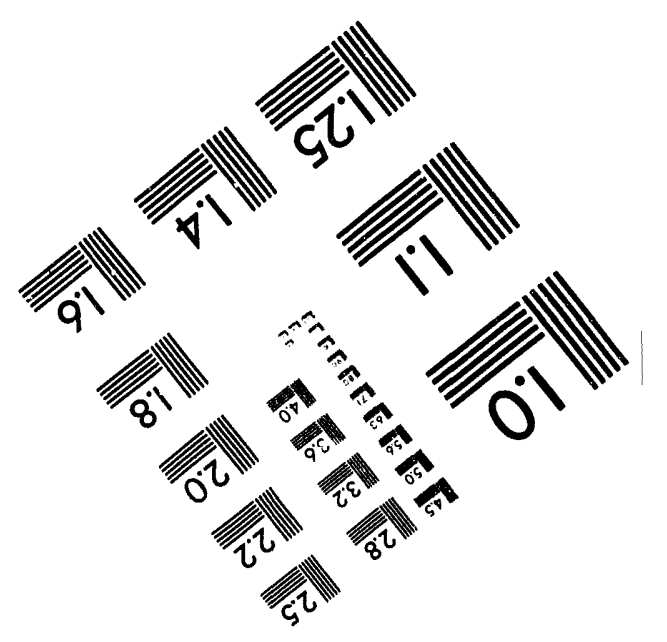



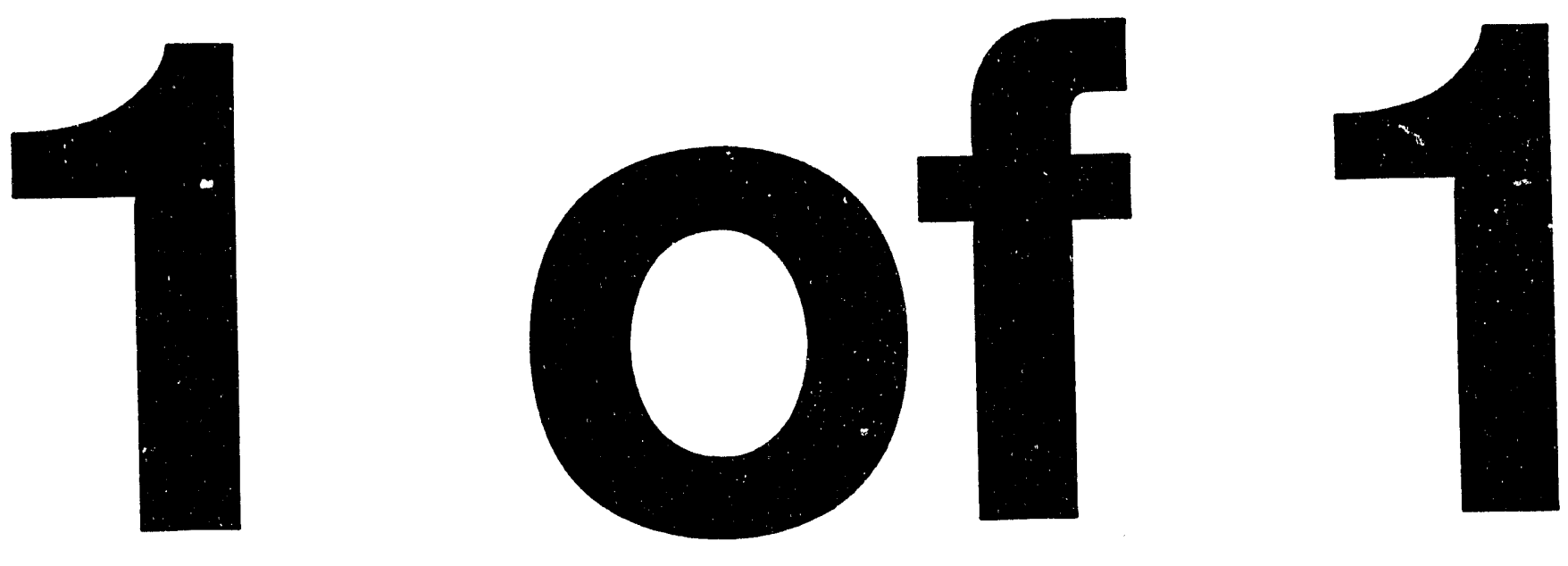


\title{
CONF-9206202--8
}

\section{FIRST PRINCIPLES THEORY OF DISORDERED ALLOYS AND ALLOY PHASE STABILITY}

\author{
G. M. Stocks *, D. M. C. Nicholson *, W. A. Shelton ', \\ B. L. Györffy ", F. J. Pinski \&, D. D. Johnson ', J. B. Staunton ॥, \\ B. Ginatempo **, P. E. A. Turchi "t, M. Sluiter ${ }^{\dagger t}$
}

June 5, 1993

\section{Introduction}

Almost immediately after the discovery of quantum mechanics, physicists and metallurgist began the task of trying to understand the origins of alloy phase stability in terms of the underlying electronic structure. Hume-Rothery [1] used the chemical concepts of electronegativity and atomic size supplemented with the solid state physics notion of electron to atom ratio to formulate a set of rules that could be used to rationalize the complex array of phases that form when two elemental metals are mixed to form an alloy. The Hume-Rothery rules and their progeny [2], [3] have proved very successful in providing metallurgists and alloy designers with a basis for understanding alloy phase stability.

In recent years however, building on a constructive interplay between advances in basic theory, advances in computational techniques, and the availability of increasingly powerful computers, the ad-hoc and semi-empirical approaches are being supplemented by fully first principles methods. In these methods, no apriori attempt is macie to identify the important physical mechanisms, rather, the system is viewed as a collection of nuclei and electrons. The major advantage of such an approach is that predictions of properties and identification of the mechanism that give rise to phase transformations do not depend on input parameters, rather, they are consequences of the properties of the underlying electronic structure responsible for metallic cohesion. The obvious disadvantage of this approach is that it is very difficult to implement in all but relatively simple situations.

Almost of necessity any $a b$ initio theory of alloy phase stability must include three key elements: firstly, a theory of the many-electron interactions that is of sufficient ac-

* Metals and Ceramics Division, Oak Ridge National Laboratory, P.O. Box 2008-6114, Oak Ridge, TN 37831, USA.

†Engineering Physics and Mathematics Division, Oak Ridge National Laboratory, P.O. Box 20086367, Oak Ridg*, TN, 37831, USA.

${ }^{\prime}$ H. H. Wills Physics Laboratory, University of Bristol, Bristol BS8 1TL, UK.

Department of Physics, University of Cincinnati, Cincinnati, OH 45221, USA.

Sandia National Laboratories, Livermore, CA 94551-0969, USA.

"Department of Physics, University of Warwick, Coventry CV4 7AL, UK.

- Istituto di Fisica Teorica, Universta di Messina, Messina, Italy.

${ }^{\dagger t}$ Lawrence Livermore National Laboratory, Livermore, CA 94550, USA. 
curacy to allow for the calculation of the small energy differences between different alloy phases; secondly, a method for implementing this theory for the types of phases encountered in alloy phase diagrams, pure elemental metals, ordered intermetallic compounds, substitutionally disordered alloys, and, ultimately, the liquid state; finally, techniques for including the statistical mechanics of compositional rearrangements that occur at finite temperature and are responsible for the most important entropic contribution to the free energy of the disordered solid solution phases.

For the first, the local density approximation (LDA) to density functional theory (DFT) [4][5][6] provides a basis for obtaining energetics to sufficient relative precision to allow one to ask questions of metallurgical interest e.g. calculation of the small energy changes associated with allotropic transformations and intermetallic compound formation. For the second, modern electronic structure methods allow the equations of LDA-DFT to be solved to a very high level of accuracy for systems with underlying periodicity. However, these methods are still limited to situations in which the basic unit cell contains a small number of atoms, typically a few tens, ideally $<20$. Thus, disordered solid solution phases present a particular problem since there in no underlying small unit cell asd experiment measures thermodynamic averages. It was for calculating these averages that the so called coherent potential approximation (CPA) was developed [7][8]. The Korringa-Kohn-Rostoker (KKR)-CPA, that will form the underpinning for these lectures, is the first principles implementation of the basic CPA idea [9][10][11] [12] within LDA.

Because of the limitations imposed by modern electronic structure methods as to the size of systems that can reasonable handled, it is at the third element, of making the link between the LDA based electronic structure methods and the statistical mechanics of compositional rearrangements, that the difficulty in obtaining a first principles theory of phase stability lies. In recent years several distinct approaches have been developed for making this connection.

In one class of methods the energetics obtained from first principles calculations are mapped onto a generalized Ising model which is then used in connection with either the Monte Carlo method [13][14] or the cluster variation method (CVM) [15] to treat the statistical mechanics of compositional rearrangements. In the Connolly-Williams method [16][17] the interactions are obtained by mapping the first principles energetics of a set of small unit cell ordered compounds to parameters of a generalized Ising model. In the generalized perturbation method (GPM) [18] [19] the Ising model interactions are obtained by expanding the electronic energy about the disordered state which is treated using the LDA-KKR-CPA method.

An alternative approach that does not require the electronically mediated interaction be mapped onto an effective Ising model is the mean field concentration functional (MF-CF) method of Gyorffy and Stocks [20]. In the MF-CF method, the electronically mediated quantum mechanical nature of the interactions is kept all the way through. Clearly this cannot be done without serious approximation. In the MF-CF method there are two major approximations. Firstly, statistical mechanics is treated only within mean field theory. Secondly, the electronic structure of the disordered phase is treated using the LDA-KKR-CPA.

The lectures of Dr. Zunger will cover the Connolly-Williams [16][17] approach. In this method, the results of first principles calculations of the energetics of a set of, small unit cell, ordered compounds are used to fit the parameters of a generalized Ising model. The advantages of such and approach are twofold. Firstly, the calculations of the energetics of the ordered compounds can be done very accurately. Secondly, once the interaction parameters for pairs, triplets eıc. are known it is relatively straight forward to calculate the thermodynamic properties of the system using either Monte 
Carlo methods or the CVM. The difficulty with such an approach is that complex electronically mediated interactions are mapped onto an effective classical Hamiltonian. Unfortunately, there is no apriori guarantee that such a procedure is either unique or rapidly convergent. In addition, since the parameters are extracted from calculations on small unit cell systems, there is possible that the interactions contain contributions (e.g. from the Madelıng energy) that will excessively favor such structures with respect to the disordered phase.

In these lecture notes we shall review the LDA-KKR-CPA method for treating the electronic structure and energetics of random alloys and the MF-CF and GPM theories of ordering and phase stability that have been built on the LDA-KKR-CPA description of the disordered phase. Thus, we take the point of view that much can be learned about metallic alloys by first studying the electronic structure and energetics of ideal random solid solutions, which, for entropic reasons, are the natural high temperature solid state phases and then to investigate their instabilities to the either phase separation or to the formation of specific ordered phases. We shall stress that a direct connection can often be made between specific features in the electronic structure associated with the random solid solution and the driving mechanisms behind specific ordering phenomena. Consequently, our understanding of phase stability will be underpinned by the same electronic structure that is responsible for determining the residual resistivity and other properties of the disordered phase and that can be experimentally verified using optical spectroscopies, positron annihilation and other probes.

These lecture notes are structured as follows. In section 2 we lay out the basic LDA-KKR-CPA theory of the electronic structure and energetics of random alloys and some examples of its applications to the electronic structure and energies of random alloys are presented. In section 3 we review the progress that has been made over the last few years in understanding the mechanisms behind specific ordering phenomena observed in binary solid solutions based on the MF-CF and GPM theories of ordering and phase stability. We will give examples of a variety of ordering mechanisms: Fermi surface nesting, band filling, off diagonal randomness, charge transfer, size difference or local strain fluctuations, and magnetic effects. In each case we will try to make the link between the specific ordering phenomenon and the underlying electronic structure of the disordered phase. In section 4 we will review the results of some recent calculations on the electronic structure of $\beta$-phase $\mathrm{Ni}_{c} \mathrm{Al}_{1-c}$ alloys using a version of the LDA-KKR$\mathrm{CPA}$ codes that has been generalized to systems having complex lattices. In section 5 we provide a few concluding remarks.

\section{Theory of Random Substitutional Alloys}

\subsection{LDA-KKR-CPA}

The LDA-KKR-CPA method for calculating the energy and other properties of random solid solution alloys rests on three theoretical developments: the local density approximation to density functional theory, multiple scattering theory for solving the effective single particle Schrödinger equation that is at the heart of the LDA-DFT self- consistent field equations, and the coherent potential approximation for treating the effects of disorder on the electronic structure i.e. for accomplishing the task of configuracional averaging inherent in the calculation of observables. 


\subsubsection{Local Density Approximation and Random Alloys}

Density functional theory (DFT) is, in principle, an exact method for calculating the energetics of an electron system in the field of the atomic nuclei [4],[5], [21], [22], [6]. The central result of DFT is that the total ground state energy, $E[\rho]$, of a system of electrons in the presence of the external field provided by the nuclei is a unique functional $E[\rho]=T[\rho]+U[\rho]+E_{x c}[\rho]$ of the electron density, $\rho(\vec{r})$, where $T[\rho], U[\rho]$ and $E_{x c}[\rho]$ are the kinetic, potential and exchange correlation energies respectively. Furthermore, $E[\rho]$ takes on its minimum value for the correct ground state $\rho(\overrightarrow{\mathbf{r}})$. This minimum principle taken together with the constraint $\int_{\infty} d^{3} r \rho(r)=N$, the total number of electrons in the system leads to a set of self-consistent field equations whose solution yield the ground state charge density and hence the ground state energy. These basic equations of DFT are made into a practical computational method by making the local density approximation (LDA) in which the unknown, but exact, exchange correlation functional for the inhomogeneous interacting electron gas appropriate to the solid is approximated, at each point in space, $\overrightarrow{\mathbf{r}}$, by the exchange correlation functional, $E_{x c}^{L D}[\rho]$, appropriate to an interacting but homogeneous electron gas having the density found at that point.

Given the specification of a solid in terms of a set of atomic positions, $\left\{R_{i}\right\}$, and corresponding nuclear charges, $\left\{Z_{i}\right\}$, of the atoms occupying these sites, the practical applications the LDA involves the solution of a set of Hartree like, Kohn-Sham selfconsistent field equations that take the form

$$
\left[-\nabla^{2}+v_{e f f}\left(\overrightarrow{\mathbf{r}} ; \rho\left(\overrightarrow{\mathbf{r}} ;\left\{R_{i}\right\} ;\left\{Z_{i}\right\}\right)\right)\right] \psi_{n}(\overrightarrow{\mathbf{r}})=\epsilon_{n} \psi_{n}(\overrightarrow{\mathbf{r}})
$$

where the crystal potential $v_{\text {eff }}\left(\overrightarrow{\mathbf{r}} ; \rho\left(\overrightarrow{\mathbf{r}} ;\left\{R_{i}\right\} ;\left\{Z_{i}\right\}\right)\right)$ takes the form

$$
v_{e f f}(\overrightarrow{\mathbf{r}})=\sum_{i} \frac{-2 Z_{i}}{\left|\overrightarrow{\mathbf{r}}-\overrightarrow{\mathbf{R}_{i}}\right|}+2 \int \frac{d \overrightarrow{\mathbf{r}} \rho(\overrightarrow{\mathbf{r}})}{\left|\overrightarrow{\mathbf{r}}-\overrightarrow{\mathbf{r}^{\prime}}\right|}+v_{x c}(\overrightarrow{\mathbf{r}} ; \rho(\overrightarrow{\mathbf{r}}))
$$

and where $\rho\left(\vec{r}_{;}\left\{R_{i}\right\} ;\left\{Z_{i}\right\}\right)$ is given in terms of the eigen-solutions of eq. 1 as

$$
\rho\left(\overrightarrow{\mathbf{r}} ;\left\{R_{i}\right\} ;\left\{Z_{i}\right\}\right)=\sum_{n}\left|\psi_{n}(\overrightarrow{\mathbf{r}})\right|^{2} f\left(\epsilon_{n}-\mu\right)
$$

where $f\left(\epsilon_{n}-\mu\right)$ is the Fermi function. For pure metals and ordered intermetallic compounds where $\left\{R_{i}\right\}$ and $\left\{Z_{i}\right\}$ define an underlying translational symmetry the LDA equations can be solved by any one of a number of band theory methods which use standard iterative techniques to make $\rho\left(\vec{r}_{;}\left\{R_{i}\right\}_{;}\left\{Z_{i}\right\}\right)$ self-consistent with $v_{e f f}\left(\overrightarrow{\mathbf{r}} ; \rho\left(\overrightarrow{\mathbf{r}}_{i}\left\{R_{i}\right\} ;\left\{Z_{i}\right\}\right)\right)$.

Unfortunately, the application of LDA-DFT to substitutionally disordered alloys is not a straight forward matter. Although, in a substitutional disordered alloy, the atoms can still be though of as occupying an underlying periodic lattice the occupancy of those lattice sites by the various atom types is random. For a binary $A_{c} B_{(1-c)}$ alloy we can specify the occupancy of a give lattice site $\vec{R}_{i}$ by a random variable $\xi_{i}$ that takes values $\xi=1$ and $\xi=0$ according as the site is occupied by the $A$ or $B$ atomic species. This is pictured schematically in fig. 1. The electron density and hence the effective one electron potential entering the Kohn-Sham equations then become functions, $\rho\left(\overrightarrow{\mathbf{r}} ;\left\{R_{i}\right\} ;\left\{\xi_{i}\right\}\right)$ and $v_{e f f}\left(\overrightarrow{\mathbf{r}} ; \rho\left(\overrightarrow{\mathbf{r}} ;\left\{R_{i}\right\} ;\left\{\xi_{i}\right\}\right)\right)$ of the set $\left\{\xi_{i}\right\}$ of occupation variables that define a specific alloy configuration.

There are two major reasons why these equations cannot be handled in a direct manner. Firstly, it is not possible to solve the LDA self-consistency equations for an arbitrary configuration that has no translational symmetry. Secondly, computation of 


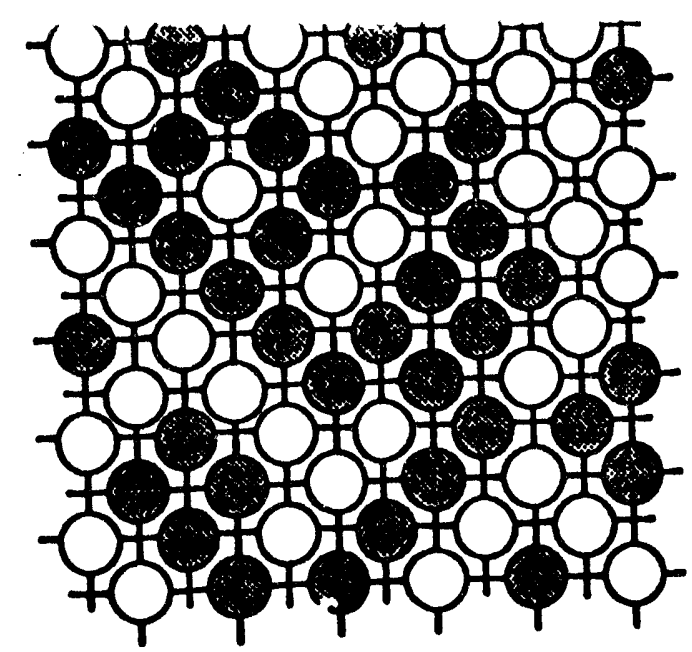

Figure 1: Schematic representation of a disordered $A_{c} B_{1-c}$ binary alloy. The $A$ and $B$ atoms randomly occupy the sites of an underlying periodic lattice.

observables involves the further step of averaging over the ensemble of configurations. For the configurationally averaged total energy, $\vec{E}$, this implies

$$
\bar{E}=\sum_{\left\{\xi_{i}\right\}} P\left(\left\{\xi_{i}\right\}\right) E_{0}^{\mathrm{LDA}}\left(\left\{\xi_{i}\right\}\right)
$$

where, in the Born-Oppenheimer approximation, the probability distribution, $P\left(\left\{\xi_{i}\right\}\right)$, is given by

$$
P\left(\left\{\xi_{i}\right\}\right)=\frac{1}{Z} e^{-\beta E_{0}^{\mathrm{LD \Lambda}}\left(\left\{\xi_{i}\right\}\right)}
$$

with

$$
Z=\sum_{\left\{\xi_{i}\right\}} e^{-\beta E_{0}^{\mathrm{LDA}}\left(\left\{\xi_{i}\right\}\right)}
$$

For a homogeneously random alloy $P\left(\left\{\xi_{i}\right\}\right)$, is given by

$$
P\left(\left\{\xi_{i}\right\}\right)=\prod_{i} p\left(\xi_{i}\right)
$$

where for a $A_{c} B_{(1-c)}$ binary solid solution the site probability, $p\left(\xi_{i}\right)$, may be parameierised in terms of the concentration $c$ of the $A$ species as

$$
p\left(\xi_{i}\right)=c \xi_{i}+(1-c)\left(1-\xi_{i}\right)
$$

Clearly, for a random alloy the ensemble of configurations is prohibitively large.

In the gedanken scheme outlined above the procedure is clear, first solve the LDA equations making $\rho\left(\overrightarrow{\mathbf{r}} ;\left\{R_{i}\right\} ;\left\{Z_{i}\right\}\right)$ self-consistent with $v_{\text {efs }}\left(\overrightarrow{\mathbf{r}} ; \rho\left(\overrightarrow{\mathbf{r}} ;\left\{R_{i}\right\} ;\left\{Z_{i}\right\}\right)\right)$, compute the total energy, then average. In the LDA-KKR-CPA method this direct approach is avoided by a subtle inversion of the self consistency and averaging processes that makes the partially averaged electron densities $\langle\rho(\overrightarrow{\mathbf{r}})\rangle_{i, \alpha}$ self-consistent with the partially averaged single site potential $\left.\left\langle v_{i}\left(\overrightarrow{\mathbf{r}}-\overrightarrow{\mathbf{R}}_{i} ; \rho(\overrightarrow{\mathbf{r}})\right]\right)\right\rangle_{i, \alpha}$. These partial averages are define suc. that $i^{\text {th }}$-site is occupied by $\alpha^{\text {th }}$-species whilst all other sites are averaged over. The KKR-CPA method is then used to calculate $\langle\rho(\overrightarrow{\mathbf{r}})\rangle_{i, \alpha}[23]$. 


\subsubsection{The KKR-CPA Method for Electronic Structure of Random Alloys}

In outlining the KKR-CPA method, rather than dealing with eq. 1 , it is necessary to deai with the corresponding equation for the single particle Green function $G(\overrightarrow{\mathbf{r}}, \overrightarrow{\mathbf{r}} ; \epsilon)$

$$
\left[-\nabla^{2}+\sum_{n} v_{n}\left(\overrightarrow{\mathbf{r}}-\overrightarrow{\mathbf{R}}_{n} ; \xi_{n}\right)-\epsilon\right] G\left(\overrightarrow{\mathbf{r}}, \overrightarrow{\mathbf{r}}^{\prime} ; \epsilon\right)=\delta\left(\overrightarrow{\mathbf{r}}-\overrightarrow{\mathbf{r}}^{\prime}\right)
$$

where $v_{n}\left(\overrightarrow{\mathbf{r}}-\overrightarrow{\mathbf{R}}_{n} ; \xi_{n}\right)$ is the effective potential associated with the $n^{\text {th }}$-site. We shall associate this with the single site partial average potential $\left\langle v_{i}\left(\overrightarrow{\mathbf{r}}-\overrightarrow{\mathbf{R}}_{i} i[(\overrightarrow{\mathbf{r}})]\right)\right\rangle_{i, a}$, more specifically with the KKR-CPA approximation to it $\bar{v}_{n}\left(\overrightarrow{\mathbf{r}}-\overrightarrow{\mathbf{R}}_{n} ; \xi_{n}\right)$. Implicit in this is the assumption that there are only $\alpha$ of these potentials i.e. that the effective potential that an electron feels at a given site depends only on the occupancy of that site and the overall composition and does not depend on the local configuration of surrounding sites. We also assume that it is sufficient to approximate the full non-spherical potential by a muffin-tin form.

Given a set of effective single site potentials the KKR-CPA provides a direct method for calculating the partially averaged Green function $\langle G(\overrightarrow{\mathbf{r}}, \overrightarrow{\mathbf{r}} ; \epsilon)\rangle_{i, \alpha}[23]$ [24] from which the partially averaged electron densities $(\rho(\overrightarrow{\mathbf{r}})\rangle_{i, \alpha}$ required by the self-consistency procedure can be obtained [24]

$$
\langle\rho(\overrightarrow{\mathbf{r}})\rangle_{i, \alpha}=-\frac{2}{\pi} \int f(\epsilon) \Im\langle G(\overrightarrow{\mathbf{r}}, \overrightarrow{\mathbf{r}} ; \epsilon)\rangle_{i, \alpha} d \epsilon .
$$

where $\Im$ indicates the imaginary part. A detailed derivation of the basic equations of the KKR-CPA Green function based on multiple scattering theory was given by Faulkner and Stocks [24]. Here we will just quote enough of the results to provide the necessary basis for the remainder of these notes.

According to Faulkner and Stocks [24] the Green function for an arbitrary array of non-overlapping muffin-tin potentials corresponding to some specific configuration of a random alloy can be written as

$$
G\left(\overrightarrow{\mathbf{r}}, \overrightarrow{\mathbf{r}}^{\prime} ; \epsilon\right)=\sum_{L L^{\prime}}\left[Z_{L}^{n}\left(\overrightarrow{\mathbf{r}}_{n} ; \epsilon\right) \tau_{L L_{1}}^{n m}(\epsilon) Z_{L^{\prime}}^{m}\left(\overrightarrow{\mathbf{r}}_{m}^{\prime} ; \epsilon\right)-Z_{L}^{n}\left(\overrightarrow{\mathbf{r}}_{n} ; \epsilon\right) J_{L}^{n}\left(\overrightarrow{\mathbf{r}}_{n}^{\prime} ; \epsilon\right) \delta_{n m} \delta_{L L^{\prime}}\right]
$$

where $Z_{L}^{n}\left(\overrightarrow{\mathbf{r}}_{n} ; \epsilon\right)$ and $J_{L}^{n}\left(\overrightarrow{\mathbf{r}}_{n}^{\prime} ; \epsilon\right)$ are the regular and irregular solutions of the Schrödinger equation for a single muffin-tin scatterer with the vector $\vec{r}_{n}=\overrightarrow{\mathbf{r}}-\overrightarrow{\mathbf{R}}_{n}$ being measured relative the center of the $n^{\text {th }}$ muffin-tin sphere. In eq. $11 \tau_{L L,}^{n m}(\epsilon)$ is the scattering path matrix [25]

$$
\tau_{L L}^{n m}(\epsilon)=t_{n, L}(\epsilon) \delta_{n m} \delta_{L L^{\prime}}+\sum_{n, m \neq p} \sum_{L^{\prime \prime}} t_{n, L}(\epsilon) g_{L L^{\prime \prime}}^{n p}(\epsilon) \tau_{L^{\prime \prime} L}^{p m}(\epsilon)
$$

where $g_{L L^{\prime \prime}}^{n p}$ are the angular momentum matrix elements of the free particle propagator connecting sites $n$ and $p$. The scattering path matrix is the generalization to many scatterers of the sih.bie site $t$-matrix, $t_{n, L}$. Whereas $t_{n, L}$ converts an incoming partial wave in angular momentum channel $L$ into an outgoing scattered wave for a single muffin-tin potential, $\tau_{L L}^{n m}(\epsilon)$ converts the incoming $L-t h$ partial wave at site $m$ into ihe outgoing $L^{\prime}-t h$ scattered partial wave at site $n$ in the presence of all of the other scatterers.

Clearly, in eq. 11, by focusing on the site diagonal Green function for the $n^{\text {th }}$ site, holding the occupancy of this site at the $\alpha$-th-species, and averaging over the remaining sites yields the partially averaged Green function $\langle(\vec{r}, \overrightarrow{\mathbf{r}} ; \epsilon)\rangle_{i, \alpha}$ that we require. The result is

$$
\langle G(\overrightarrow{\mathbf{r}}, \overrightarrow{\mathrm{r}} ; \epsilon)\rangle_{i, \alpha}=\sum_{L L^{\prime}}\left[Z_{L}^{n, \alpha}\left(\overrightarrow{\mathrm{r}}_{n} ; \epsilon\right)\left\langle\tau_{L L^{\prime}}^{n n}\right\rangle_{n, \alpha} Z_{L^{\prime}}^{n, \alpha}\left(\overrightarrow{\mathrm{r}}_{n}^{\prime} ; \epsilon\right)-Z_{L}^{n, \alpha}\left(\overrightarrow{\mathrm{r}}_{n} ; \epsilon\right) J_{L}^{n, \alpha}\left(\overrightarrow{\mathrm{r}}_{n}^{\prime} ; \epsilon\right) \delta_{L L^{\prime}}\right]
$$


Thus, the problem of finding the partially averaged single site electron density nas been reduced to finding a theory for the partially averaged scattering path matrix $\left(\tau_{L L}^{n n}\right\rangle_{n, \alpha}$. This is what the KKR-CPA is set up to do.

The essence of the KKR-CPA is to approximate the scattering properties of the disordered array of real scattering centers, each characterized by a single site t-matrix $t_{\alpha}$, by the scattering properties of an ordered array of effective scatterers characterized by some effective t-matrix, $t_{C}(\epsilon)$, and then to choose $t_{C}(\epsilon)(\epsilon)$ such that it gives the best approximation to the real system. In the KKR-CPA best is defined in the sence that this approximation is the best that can be achieved whilst considering only single-site partial averages. As such the KKR-CPA specifically ignores effects associated with specific local configurations.

The scattering path matrix, $\tau^{C, n n}(\epsilon)$, for an array of effective scatterers is simply given by the site diagonal element of eq. 12 where all the $t$-matrices are $t_{C}(\epsilon)$ 's. Since, the underlying lattice is periodic the resulting equation for the ordered array of effective scatterers can be solved using be lattice Fourier transforms to yield

$$
\tau^{C, n m}(\epsilon)=\int d \vec{k} e^{\vec{k} \vec{R}_{n m}}\left[t_{C}^{-1}(\epsilon)-G(\vec{k} ; \epsilon)\right]^{-1}
$$

Where $\tau^{C, n n}(\epsilon), t_{C}^{-1}(\epsilon)$ and $G(\vec{k} ; \epsilon)$ are matrices in $\left(L L^{\prime}\right)$ and the latter are the KKRstructure constants [23]. $\vec{R}_{n m}$ is the vector connecting sites $n$ and $m$. If we now replace the effective t-matrix at site $n$ by the t-matrix for the $\alpha^{\text {th }}$ species the corresponding site diagonal scattering path matrix $\tau^{\alpha, n n}(\epsilon)$ describes the scattering from an $\alpha$ impurity embedded in the effective medium.

The CPA is then obtained by requiring that replacing a single effective scatterer at some site $n$ by a real scatterer produces no further scattering when averaged over all of the species. Mathematically this statement reduces to the requirement

$$
\sum_{\alpha} c_{\alpha} \tau^{\alpha, n n}(\epsilon)=\tau^{C, n n}(\epsilon)
$$

where $\tau^{\alpha, n n}(\epsilon)$ is given by the solution to the single impurity problem

$$
\tau^{\alpha, n n}(\epsilon)=\left[1+\tau^{C, n n}(\epsilon)\left(t_{C}^{-1}(\epsilon)-t_{\alpha}^{-1}(\epsilon)\right)\right]^{-1} \tau^{C, n n}(\epsilon)
$$

Taken together eqs.15,16, and 14 form a self-consistency condition that determines the effective KKR-CPA scatterer. We refer to these equations collectively as the KKRCPA equations.

Finally, making the associations

$$
\left\langle\tau^{n n}\right\rangle_{n, \alpha} \Rightarrow \tau^{\alpha, n n}(\epsilon)
$$

and

$$
\langle\rho(\overrightarrow{\mathbf{r}})\rangle_{n, a} \Rightarrow \bar{\rho}_{n}^{\alpha}(\overrightarrow{\mathbf{r}})
$$

in eq. 13 yields the KKR-CPA approximation, $\bar{\rho}_{n}^{\alpha}(\overrightarrow{\mathbf{r}})$, to the partially averaged single site electron density that we require for the LDA self-consistency step. $\bar{\rho}_{n}^{a}(\vec{x})$ is given by

$$
\bar{\rho}^{-\alpha}(\overrightarrow{\mathbf{r}})=\frac{-2}{\pi} \Im \int_{-\infty}^{\epsilon} d \epsilon\left[\sum_{L L^{\prime}}\left[Z_{L}^{n, \alpha}\left(\vec{r}_{n} ; \epsilon\right) \tau_{L L^{\prime}}^{\alpha, n n}(\epsilon) Z_{L^{\prime}}^{n, \alpha}\left(\vec{r}_{n}^{\prime} ; \epsilon\right)-Z_{L}^{n, \alpha}\left(\vec{r}_{n} ; \epsilon\right) J_{L}^{n, \alpha}\left(\vec{r}_{n} ; \epsilon\right) \delta_{L L^{\prime}}\right]\right] .
$$


Before closing this section we note that a couple of other quantities of interest can be obtained straight forwardly from the partially averaged Green function. The single site density of states is given by

$$
\bar{n}^{-\alpha}(\epsilon)=\frac{-2}{\pi \Omega} \Im \int_{\Omega_{n}} d \vec{r}_{n}\left[\sum_{L L^{\prime}}\left[Z_{L}^{n, \alpha}\left(\vec{r}_{n} ; \epsilon\right) \tau_{L L^{\prime}}^{C, n n}(\epsilon) Z_{L}^{n, \alpha}\left(\vec{r}_{n} ; \epsilon\right)-Z_{L}^{n, \alpha}\left(\vec{r}_{n} ; \epsilon\right) J_{L}^{n, \alpha}\left(\vec{r}_{n} ; \epsilon\right) \delta_{L L^{\prime}}\right]\right] .
$$

from which the configurationally averaged total density of states can be obtained by performing the final average over the species

$$
\bar{n}(\epsilon)=\sum_{\alpha} c_{\alpha} \overline{n^{\alpha}}(\epsilon)
$$

The Fermi energy can be obtained from the charge neutrality condition

$$
\sum_{\alpha} c_{r} Z_{\alpha}=\int_{-\infty}^{\epsilon}{ }_{-\infty} \epsilon^{-\alpha}(\epsilon)
$$

where the lhs of eq. 22 is simply the average nuclear charge in the system, $\bar{Z}$.

The final quantity of interest for future discussions is the Bloch spectral function, $A^{B}(\overrightarrow{\mathrm{k}}, \epsilon),[24]$

$$
A^{B}(\overrightarrow{\mathbf{k}}, \epsilon)=-1 / \pi \sum_{i, j} e^{i \overrightarrow{\mathbf{k}} \cdot\left(\mathbf{R}_{i}-\mathbf{R}_{j}\right)} \int_{\Omega_{i}} \Im\left\langle G\left(\overrightarrow{\mathbf{r}}+\overrightarrow{\mathbf{R}}_{i} ; \overrightarrow{\mathbf{r}}+\overrightarrow{\mathbf{R}}_{j} ; \epsilon\right)\right\rangle d \overrightarrow{\mathbf{r}}
$$

The Bloch spectral function contains a complete description of the electronic structure of a random alloy. It is the generalization to a disordered system of the band structure of pure metals and ordered compounds. Indeed, for an ordered system it reduces to

$$
A^{B}(\vec{k}, \epsilon)=\sum_{\nu} \delta\left(\epsilon-\epsilon_{\vec{k}, \nu}\right)
$$

where $\overrightarrow{\mathbf{k}}$ is the Bloch wave-vector and $\nu$ is the band index. For disordered alloys the ORNL-DWG 91.9202
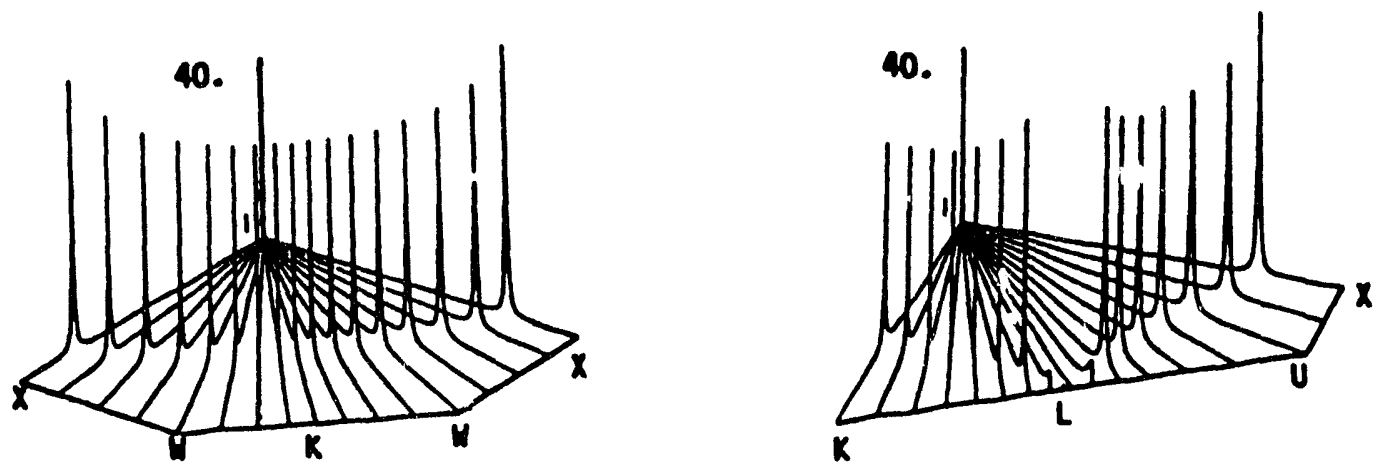

\section{C40.75Pdo.25}

Figure 2: The Bloch spectral density function $A^{B}(\overrightarrow{\mathrm{k}}, \epsilon)$ at the Fermi energy $\epsilon=\epsilon_{F}$ along various directions emanating from the center of the fcc Brillouin zone for a disordered $\mathrm{Cu}_{0.75} \mathrm{Pd}_{0.25}$ alloy.

$\delta$-function peaks of eq. 24 broaden into peaks with finite width and finite heights whose position in $\vec{k}$, $\epsilon$ space trace out the band structure. The finite widths are interpretable as finite inverse life-times if viewed in energy space or inverse mean free paths if viewed in $\vec{k}$ space. It should be stressed that while $\vec{k}$ is not a good quantum 

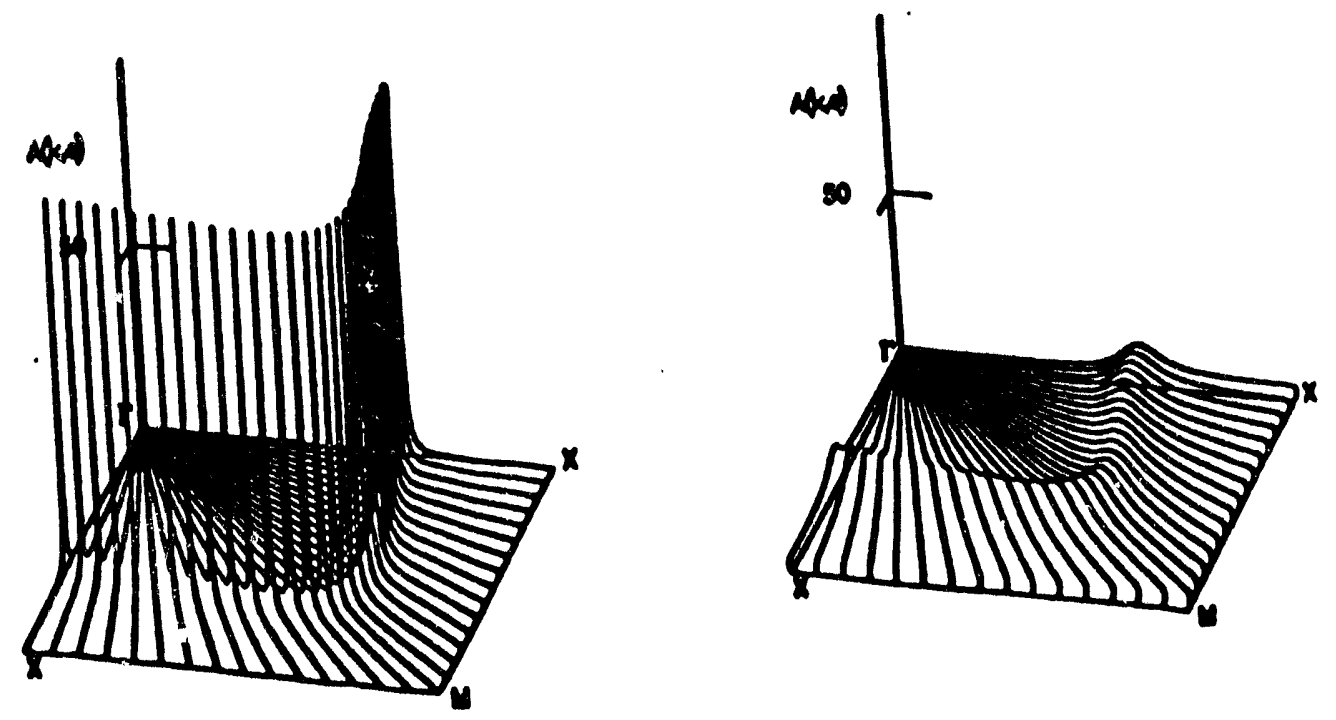

Figure 3: The Bloch spectral density function $A^{B}(\overrightarrow{\mathbf{k}}, \epsilon)$ at the Fermi energy $\epsilon=\epsilon_{F}$ along various directions emanating from the center of the bcc Brillouin zone for ordered B2 structure $\mathrm{NiAl}$ (left) and a hypothetical disordered bcc Nio.s $\mathrm{Al}_{0.3}$ alloy (right).

number for a given configuration $\left\{\xi_{i}\right\}$ it does correspond to the translational symmetry of the average lattice. Specifically, $A^{B}(\vec{k}, \epsilon)$ is periodic and has the Brillouin zone of the unoccupied lattice. As examples of systems in which the effects of disorder on the electronic structure are very different, in figs. 2 and 3 we show the Fermi energy Bloch spectral functions for disordered fcc $\mathrm{Cu}_{0.75} \mathrm{Pd}_{0.25}$ and hypothetical disordered bcc $\mathrm{Ni}_{0.5} \mathrm{Al}_{0.3}$. For $\mathrm{Cu}_{0.75} \mathrm{Pd}_{0.25}$ the effects of disorder on $A^{B}(\vec{k}, \epsilon)$ are small, the peaks are well defined, and the corresponding mean free path is long. Under such circumstances, it is possible to view the surface in $\vec{k}$-space defined by the locus of the peak positions of $A^{B}(\vec{k}, \epsilon)$ as being the "Fermi surface" of the disordered alloy. For Nio.s $A l_{0.5}$ the effects of disorder on $A^{B}(\vec{k}, \epsilon)$ are large, the weight in $A^{B}(\vec{k}, \epsilon)$ is spread throughout the Brillouin zone and it is no longer possible to speak of this alloy having a Fermi surface in any meaningful sense.

\subsubsection{Self-consistency and Total Energy}

As indicated above, given a set of effective single site potentials the KKR-CPA provides a direct method for calculating the partially averaged electron densities $\bar{\rho}^{\alpha}(\vec{r})$ that are required in order to develop a self-consistency procedure. All that now remains is to specify how the configurationally averaged single site potentials, $\vec{v}^{\alpha}(\overrightarrow{\mathbf{r}})$ are related to $\bar{\rho}^{\alpha}(\overrightarrow{\mathbf{r}})$ and then to specify how the configurationally average energy $\bar{E}$ is to be obtained from the self-consistent $\bar{\rho}^{\alpha}(\vec{r})$ and $\bar{v}^{\alpha}(\vec{r})$.

Since the KKR-CPA method is itself a mean field theory of the effects of disorder on the electronic structure, we have chosen to specify the single site potentials in a mean field approximation. Specifically, the potential on an $\alpha$ site is taken to be the exchange potential corresponding to $\vec{\rho}^{\alpha}(\vec{r})$ plus the solution of Poisson's eq 1ation for a electron density consisting of $\bar{\rho}^{\alpha}(\overrightarrow{\mathbf{r}})$ on the central site and the concentration averaged electron density $\bar{\rho}(\vec{r})=\sum_{\alpha} c_{\alpha} \bar{\rho}^{\alpha}(\vec{r})$ on all other sites. In the muffin-tin approximation this results in [26]

$$
v_{\alpha}(r)=-\frac{2 Z_{\alpha}}{r}+8 \pi \int_{0}^{r} d r^{\prime}\left(\frac{r^{\prime 2}}{r}-r^{\prime}\right) \bar{\rho}_{\alpha}\left(r^{\prime}\right)+8 \pi \int_{0}^{R_{m 1}} d r^{\prime} r^{\prime} \bar{\rho}_{\alpha}\left(r^{\prime}\right)
$$




$$
+\mu_{x c}\left(\bar{\rho}_{a}(r)\right)-\mu_{x c}\left(\bar{\rho}_{0}\right)+\frac{C}{a} \bar{\rho}_{0} \Omega_{i n t} .
$$

where $\Omega_{\text {int }}$ is the volume of the interstitial region

$$
\Omega_{\text {int }}=\Omega_{w s}-\frac{4 \pi}{3} R_{m t}^{3},
$$

and $\bar{\rho}_{0}$ is the average of the interstitial electron density

$$
\bar{\rho}_{0}=\frac{1}{\Omega_{\text {int }}} \sum_{\alpha=1}^{B} c_{\alpha}\left\{Z_{\alpha}-4 \pi \int_{0}^{R_{m t}} d r r^{2} \rho_{\alpha}(r)\right\} .
$$

It should be noted that the muffin-tin potential given by (25) for the disordered structure has the same form as that for ordered structures [27], except that the interstitial electron density $\rho_{0}$ is replaced by $\bar{\rho}_{0}$.

The LDA-KKR-CPA algorithm is now straightforward. Initial guesses of the partially averaged electron densities $\bar{\rho}^{\alpha}$ associated with each of the alloying species are used to compute initial partially averaged potential functions $\bar{v}^{\alpha}\left(\overrightarrow{\mathbf{r}}-\vec{R}_{n} ; \bar{\rho}^{\alpha}, \bar{\rho}, \bar{\rho}_{0}\right)$ which are to be used in the KKR-CPA calculation of new partially averaged electron densities. The input and output $\bar{\rho}^{\alpha}$ are compared, if they are not equal within some prescribed tolerance then a new guess of the input $\bar{\rho}^{\alpha}$ is made according to some appropriate mixing prescription and the process is repeated until self-consistency is obtained.

Once the self-consistent $\bar{\rho}^{\alpha}(\vec{r})$ have been obtained within the KKR-CPA all that remains is to calculate the configurationally averaged total energy $\bar{E}$. I In order to establish a relationship between $\bar{\rho}^{\alpha}(\overline{\mathbf{r}})$ and $\bar{E}$ we begin with the observation [12] that the grand potential $\Omega(T, V, \mu)$ is in general related to the configurationally averaged density of states through the thermodynamic identity

$$
\frac{d \Omega(T, V, \mu)}{d \mu}=-N(\mu)
$$

where $\mu$ is the electron chemical potential and $N(\mu)$ is the number of electrons

$$
N(\mu)=\int_{-\infty}^{\infty} d \epsilon \bar{n}(\epsilon) f(\epsilon-\mu)
$$

Equation 28 can be integrated to yield an expression for $\Omega$ that is linear in $\bar{n}$ that can be configurationally averaged within the CPA. Thus, the above procedure circumvents the need to form the troublesome configurational averages of the square of the charge density that occurs in the standard expression for the toial energy. The result of this procedure is

$$
\Omega=-\int_{-\infty}^{\infty} d \epsilon \bar{N}\left(\epsilon,\left\{\bar{v}_{\alpha}\right\}\right) f(\epsilon-\mu)+\int_{-\infty}^{\mu} d \mu^{\prime} \int_{-\infty}^{\infty} d \epsilon f(\epsilon-\mu) \frac{d \bar{N}\left(\epsilon,\left\{\bar{v}_{\alpha}\right\}\right)}{d \mu^{\prime}}
$$

where $\bar{N}$ is the configuration averaged integrated density of states,

$$
N(\epsilon)=\int_{-\infty}^{\epsilon} d \epsilon \bar{n}(\epsilon)
$$

The first term in eq. 30 is the familiar contribution of the eigenvalue sum plus the electron hole entropy. The remaining term is the so called double counting term written in an unfamiliar form. The important point is, once it has been decided to use the CPA, that the relationship between the electron density and integrated density of states is fixed because they are derived from the CPA Green function. Using single site 
potentials specified by eq. 25 the second term on the rhs of eq. $3 U$ can be untegrated to obtain [12] an expression for $\Omega$. Taking $T=0$ yields a configurationally averaged energy of the form

$$
\bar{E}=\sum_{\alpha} c_{\alpha} E_{J}^{\alpha}\left[\bar{\rho}^{\alpha}, \bar{\rho}, \bar{\rho}_{0}\right]
$$

where $E_{J}$ is the expression derived by Janak [27] for ordered systems for a crystal potential in the muffin-tin form, excepting that the pure metal charge densities of Janak are replaced by the configurationally averaged ones defined above. A remarkable feature of eq. 32 is, thanks to the use of CPA, that $\bar{E}$ retains the variational properties characteristic of $E[\rho]$ for pure systems. Namely

$$
\frac{\partial \bar{E}}{\partial \bar{\rho}^{\alpha}}=0
$$

Furthermore, as is the case for normal LDA-DFT, taking the variation of the potential energy $\bar{U}[\rho]$ with respect to the single site densities yields the effective potential that enters the Schrödinger equation

$$
\frac{\partial \bar{U}}{\partial \bar{\rho}^{\alpha}}=c_{\alpha} \bar{v}_{\alpha}(\overline{\mathbf{r}})
$$

These latter are clearly some of the reasons for the success of the KKR-CPA theory for the total energy [12].

\subsection{Electronic Structure and Properties of Binary Alloys}

By now there are several KKR-CPA codes in existence. They have been used to understand a large body of experimental measurements of the electronic properties of disordered solid solutions. Here we mention just few that have come out of our work. We do this to establish the overall correctness of the KKR-CPA description of the electronic structure and, more importantly, to make the point that the electronic structure provides an interpretation of experimental probes such as X-ray photoelectron spectroscopy and residual resistivity, and further, provides a basis for understanding the driving mechanisms behind ordering phenomena and alloy phase stability.

In fig. 4 we show calculated total and component densities of states and XPS spectra for two concentrations of $\mathrm{Cu}_{c} \mathrm{Pd}_{1-c}$ that were calculated by Winter et cl. [28]. For each composition, the lower left of the four frames compares the calculated XPS spectrum with experiment. It should be noted that the calculated spectra take proper account of the optical matrix elements and that this is a import feature in obtaining the ple asing agreement between theory and experiment.

$\tau_{\text {ant }}$

The residual resistivity is a particularly sensitive measure of the electronic structure of a disordered alloy since it not only depends on the topology of the Fermi surface but also on the extent to which it is smeared out by disorder. In fig. 5 we show the calculated concentration dependence of the residual resistivity of $\mathrm{Agc}_{\mathrm{g}} \mathrm{Pd}_{1-c}$ alloys obtained by Butler and Stocks [29] together with $A^{B}\left(\vec{k}, \epsilon_{F}\right)$ in two planes of the fcc Brillouin zone for the compositions $c=0.25(\mathrm{a}, \mathrm{d}), c=0.50(\mathrm{~b}, \mathrm{e})$, and $c=0.75$ $(c, f)$. The calculations of the residual resistivity are based on a simple relaxation time approximation. In theses calculations the residual resistivity is controlled by the coherence length of the electrons on the well defined $\Gamma$-centered sheet of Fermi surface which is proportional to $\vec{k}$-width of the Lorentzian like peaks. The overall agreement between theory and experiment is then a measure the quality of the treatment of disorder afforded by the KKR-CPA. 

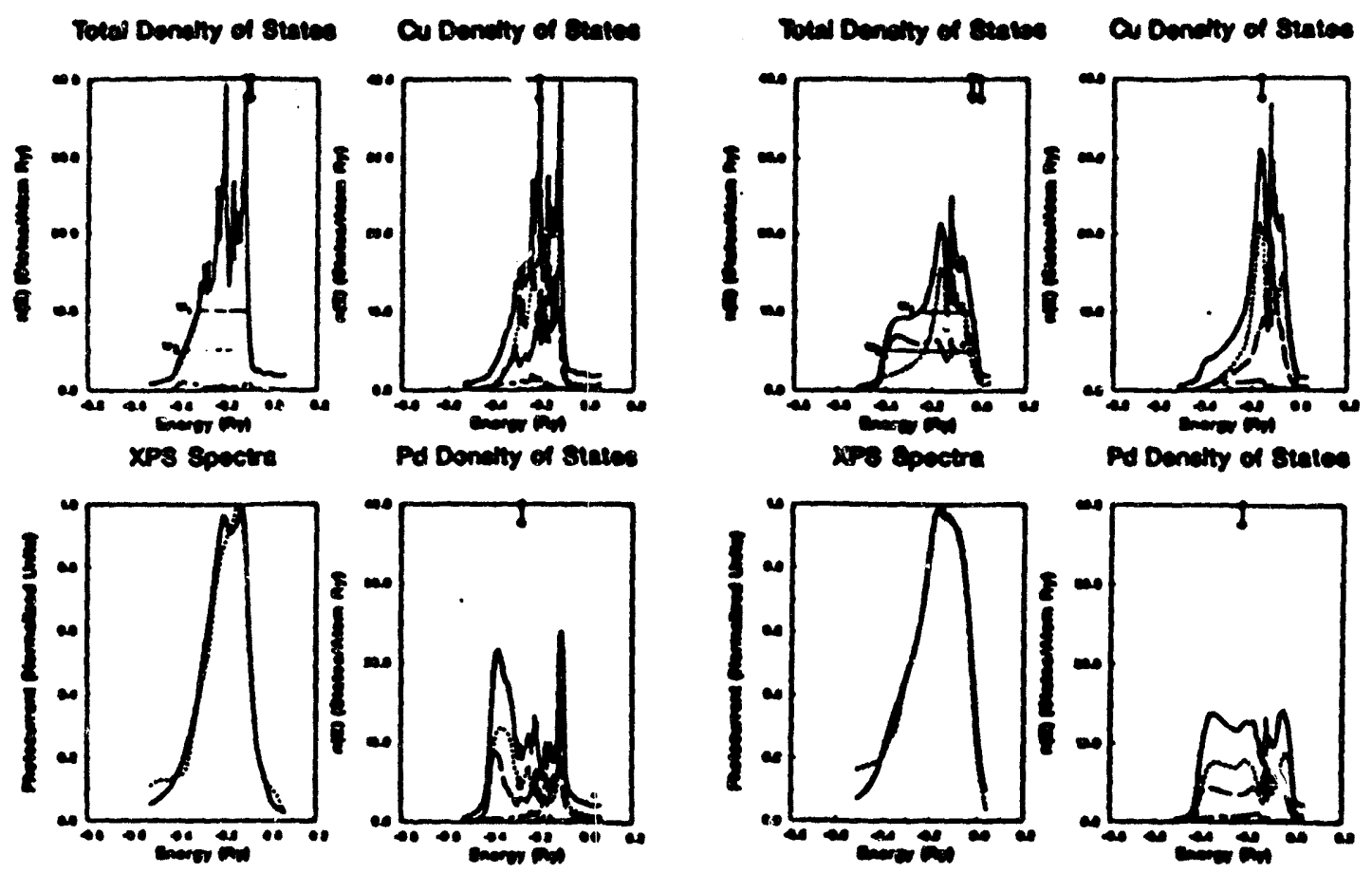

Figure 4: Total, and component densities of states and XPS spectra for disurdered $\mathrm{Cu}_{c} \mathrm{Pd}_{1-c}$ at two concentrations ( $c=0.95$ left four frames; $c=0.5$ right four frames). The experimental (dashed) and theoretic (solid) XPS spectra are compared for each composition in the lower left frames.
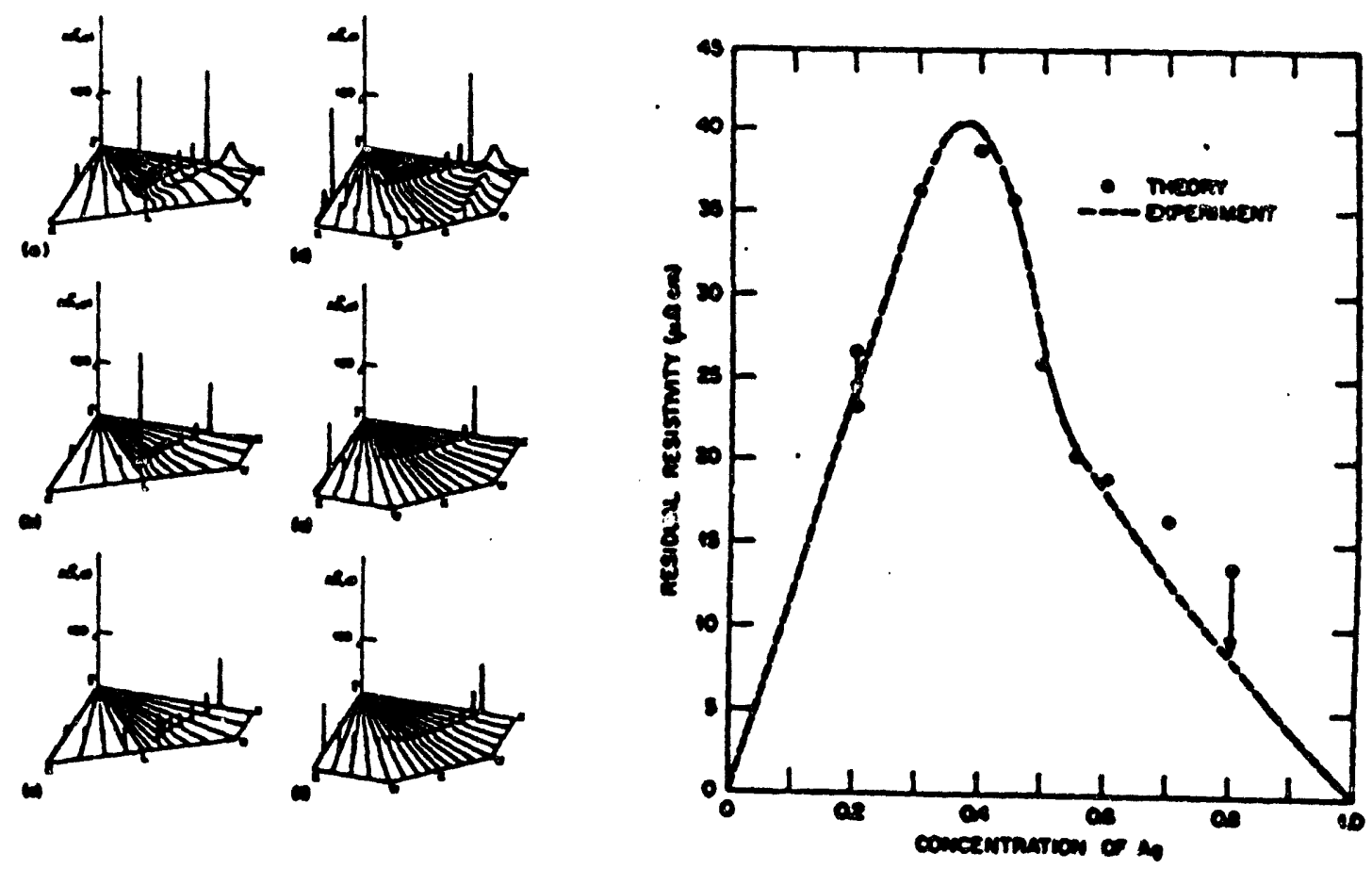

Figure 5: Fermi energy Bloch spectral functions (left frames) and calculated residual resistivity (right frame) for disordered $\mathrm{Ag}_{c} \mathrm{Pd}_{1-c}$ alloys [29]. 
The relatively large departure between theory and experiment for Ag-rich alloys results from the neglect of vertex corrections in the calculation. Subsequently, Butler [30! developed a proper Kubo formula based theory of the residual resistivity that takes account of the vertex corrections but is still based on the KKR-CPA. The results of the calculations of Swihart et al. based on Butler's formulation are shown in fig. 6 . For $A_{g c} P_{1-c}$ alloys the calculated results are in excellent agreement with experiment.

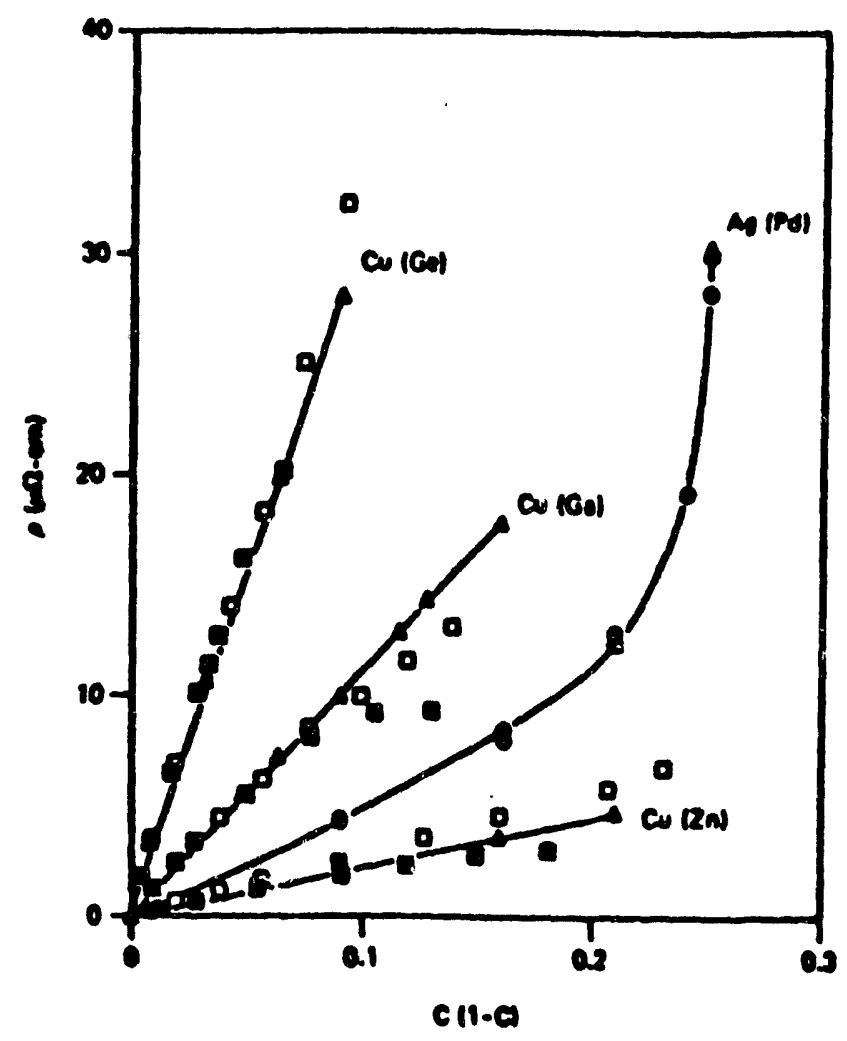

Figure 6: Calculated and measured residual resistivities for $\mathrm{Cu}$-rich $\mathrm{Cu}_{c} \mathrm{Zn}_{1-c}$, $\mathrm{Cu}_{c} \mathrm{Ga}_{1-c}, \mathrm{Cu}_{c} \mathrm{Ge}_{1-c}$, and $\mathrm{Ag}$-rich $\mathrm{Ag}_{c} \mathrm{Pd}_{1-c}$ alloys [31]. The open triangles and solid lines are the calculated values. The open symbols (squares for $\mathrm{Cu}$ alloys and circles for Ag alloys) are for cold worked samples, while the solid symbols are for annealed samples.

For the $\mathrm{Cu}$-based alloys the calculated results are in excellent agreement with the measured values for the cold worked samples. This is suggestive that the departure for the annealed samples results from the presence of chemical short range order, the effects of which are not considered in the calculations, and which is broken up by cold work.

Having pointed to some of the successes of the KKR-CPA in understanding the electronic structure of disordered alloys we now turn to the energetics.

\subsection{Energies of Mixing}

Given a theory of the total energy of the disordered state it is a relatively straightforward matter to calculate the energy of mixing $\Delta E^{\text {mix }}$. For a binary substitutional $\mathrm{A}_{c} \mathrm{~B}_{1-c}$ alloy $\Delta E^{\mathrm{mix}}$ is given by

$$
\Delta E^{\text {mix }}=\bar{E}-c E^{A}-(1-c) E^{B}
$$

where $E^{A}$ and $E^{B}$ are the ground state energies of the pure $A$ and pure $B$ metals. The way the calculations proceed is entirely standard; for a given underlying crystal 
structure, the energy is calculated as a function of lattice spacing for both pure metals and disordered alloys. The ground state energy and equilibrium lattice spacing are given, in plots of energy vs lattice constant, by the minirnum energy and corresponding lattice constant. The ground state energies are then used in eq. 35 to obtain the energy of mixing. It is perhaps worth a ccmment on the significance of the energy of mixing defined in this way since, ideally, the compositionally homogeneously disordered state to which the LDA-KKR-CPA energies correspond is only realizable at infinitely high temperature but we are evaluating it at $T=0 \mathrm{~K}$. Certainly, this energy does not have to correspond to measured enthalpies of mixing since these measurements are often made at moderate temperature. However, excepting in systems where short range order effects are particularly pronounced one would not expect them to be wildly different.

Fig. 7 shows the results of our calculations of $\Delta E^{\text {mix }}$ for the $\mathrm{Cu}_{c} \mathrm{Zn}_{1-c}$ alloy system [12] [32] for both fcc and bcc structures. Since, structural energy differences cannot be

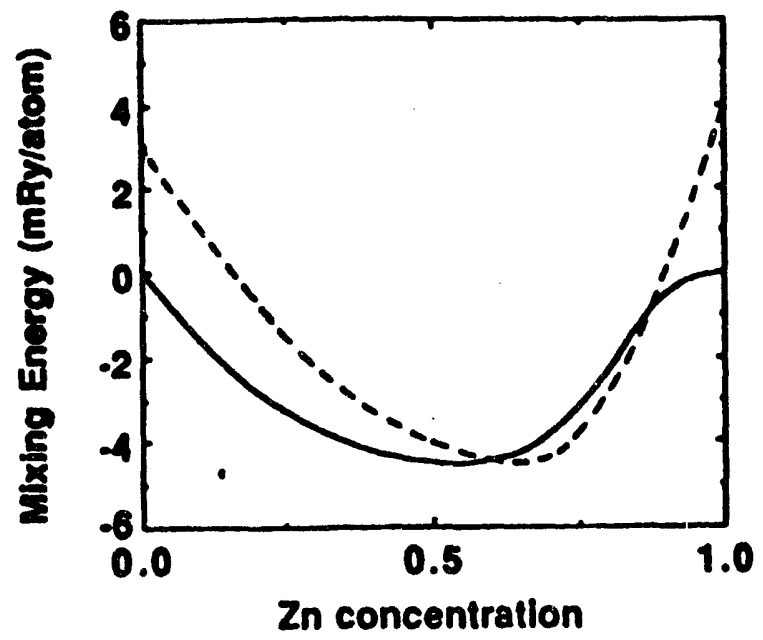

Figure 7: Concentration variation of the heats of mixing for fcc (solid line) and bcc (dashed line) $\mathrm{Cu}_{c} \mathrm{Zn}_{1-c}$ alloys [12], [32].

reliably obtained from calculations based on the muffin-tin approximation the values of $\Delta E^{\text {mix }}$ for the bcc structure show in fig. 7 are referenced to the fcc by taking taking the structural energy differences for the elemental metals from full-potential FLAPW calculations.

The negative sign of $\Delta E^{\mathrm{mix}}$ implies an ordering tendency which, indeed, is a feature of the rather complicated $\mathrm{Cu}_{c} \mathrm{Zn}_{1-c}$ phase diagram [33]. Interestingly $\Delta E^{\mathrm{mix}}$ is not strictly parabolic and this suggests a complex phase diagram. Comparison of the calculated energies of mixing in the disordered phase with available experimental measurements [34] and with those obtained from assessed phase diagrams [35] suggests that the calculated values that are approximately a half of the experimental ones. Since, as we shall see later, the calculated values produce a quite good description of the phase diagram of $\mathrm{Cu}$-rich $\mathrm{Cu}_{c} \mathbf{Z n}_{1-c}$ alloys, this discrepancy may be more apparent than real. However, recent calculations [36] of the total energy of the disordered state that attempt to go beyond mean field theory in the potential reconstruction step and include some account of charge correlations between neighboring sites suggest that this discrepancy may be due to the use of the mean field potential reconstruction and the fact that there is some small charge transfer.

In fig. 8 we show calculated energies of mixing and equilibrium lattice spacing for $\mathrm{Al}_{c} \mathrm{Ag}_{1-c}$ alloys [37]. The shape of the calculated energy of mixing curve deviates strongly from the parabolic $c(1-c)$ shape assumed in regular solution theory and is 

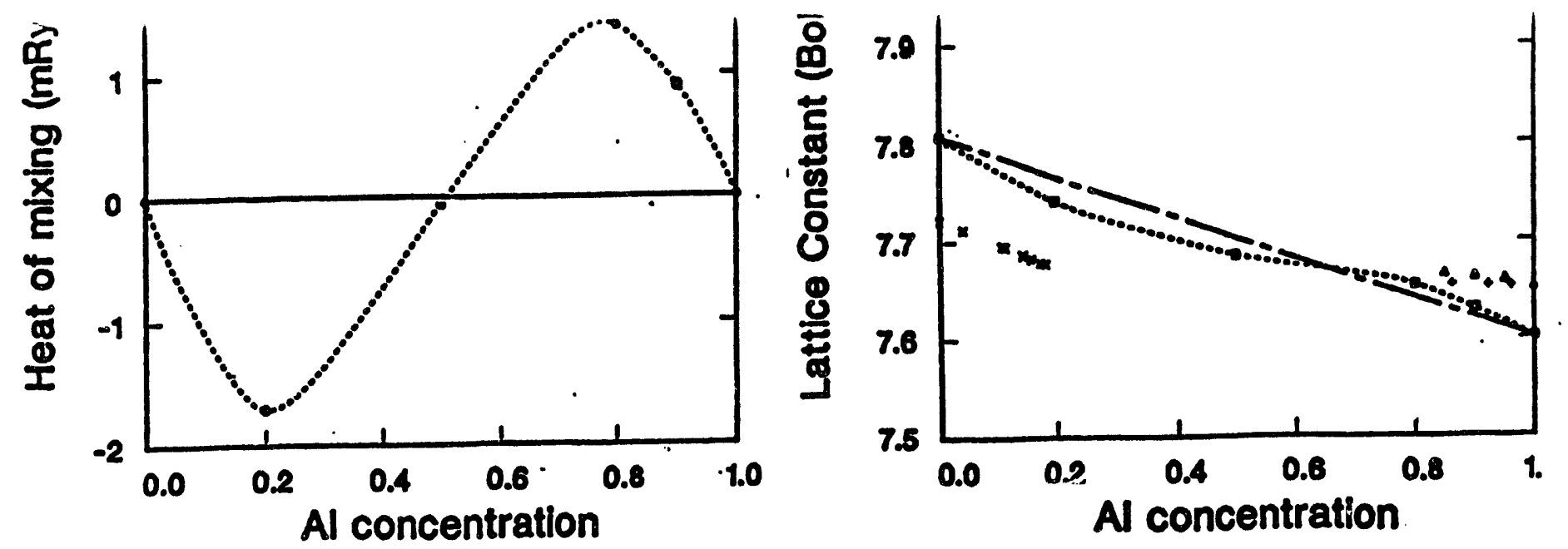

Figure 8: Calculated concentration variationt of the heats of mixing (left frame) and equilibrium lattice spacing (right frame) of $\mathrm{Al}_{c} \mathrm{Ag}_{1-c}$ alloys. In the lattice parameter plot the short-long dashed line is included to highlight the deviation of the calculated results (dashed line and squares) from Vegard's rule. The remaining symbols give selected room temperature experimental values [38].

again indicative of a complex phase diagram. The positive energies of mixing for Alrich alloy suggests that these alloys would want to phase separate, whilst the negative values of $\Delta E^{\text {mix }}$ for Ag-rich alloy suggests that these alloys would want to order at low temperature. We shall return to this observation later. The calculated concentration dependence of the equilibrium lattice spacing deviates slightly from Vegard's rule with the sign of the deviation being positive for Al-rich alloys and negative for Ag-rich. Comparison with the concentration dependence observed experimentally is made difficult by the fact that for pure $\mathrm{Ag}$ and pure $\mathrm{Al}$ the calculated equilibrium lattice constants deviate from experiment with opposite signs, however, it is the case that the sense of the deviation from Vegard's rule for the experimental results is in agreement with the calculation.

\section{Ordering and Phase Stability}

\subsection{LDA-KKR-CPA Based Theories}

Having developed a theory of the electronic structure and energetics of the ideal random solid solution we can now turn to the task of building a theory of ordering and phase stability on it. Thus, we have to address the difficult task of accounting for the statistical mechanics of concentration fluctuations within a first principles theory. In the following subsections we will briefly describe and show results for two rather different approaches, a concentration functional (CF) theory implemented within mean field theory, and the generalized perturbation method (GPM). Whilst both methods are based on the underlying LDA-KKR-CPA theory of the disordered state, the emphasis is rather different.

The mean field theory places stress on retaining the electronic interactions in their full generality. The consequences of this approach is that it is only possible to treat the statistical mechanics of concentration fluctuation within mean field theory. Thus potentially important cluster effects within the configurational entropy are neglected. However, as with most mean field theories the results of this approach not only provide important insights into the physical mechanism that drive particular orderings but also 
provide a reference from which to judge the need for including a better treatment ol the configurational entropy such as that provided by the Monte Carlo method or thi cluster variation method (CVM).

In the GPM, stress is placed on obtaining a better description on the configurational entropy than that provided by the mean field or point approximation. Consequently, the electronic interactions are mapped onto a generalized Ising model, and the electronic energy of specific configurations is partitioned between site, pairs, triplets, etc., nearest neighbors, next nearest neighbors, etc.. However, when coupled with the LDAKKR-CPA the GPM provides a first principles means by which these interactions can be calculated. Once, calculated questions of short range order and phase stability can be explored using the CVM and Monte Carlo method.

\subsection{Mean Field Concentration Functional Theory}

The first principles concentration functional thaory introduced by Gyorffy and Stocks [20] is based on an adaptation of the classical density functional theory of liquids [39] to a lattice gas model of substitutionally disordered alloys. Though a powerful device that produces a number of interesting results, the theory is much like the density functional theory of the electron gas in that without the local density approximation, which converts the theory into a practical computational method, little can be done with it. For the concentration functional method, the approximation that makes the method tractable is the mean field (MF) approximation.

The MF-CF method was set out in the original paper and has been restated several times since, both in applications to alloy ordering [40], [41] and in connection with the disordered local moment theory of ferromagnetic ordering in the $3 d$ itinerant ferromagnets [42]. Thus, we will only state the principle results.

The central result of the mean-field CF theory is that there is a mean field grand potential, $\Omega^{\mathrm{MF}}\left(T, \nu,\left\{c_{i}\right\}\right)$,

$$
\begin{aligned}
\Omega^{\mathrm{MF}}\left(T, \nu,\left\{c_{i}\right\}\right) & =\Omega^{\mathrm{CPA}}\left(\left\{c_{i}\right\}\right) \\
& +\mathrm{k}_{\mathrm{B}} \mathrm{T} \sum_{i}\left(c_{i} \ln c_{i}+\left(1-c_{i}\right) \ln \left(1-c_{i}\right)\right) \\
& -\sum_{i} \nu_{i} c_{i}
\end{aligned}
$$

that is a function of temperature, $T$, chemical potential difference, $\nu$, and all the local concentrations $\left\{c_{i}\right\}$, with $c_{i} \equiv\left\langle\xi_{i}\right\rangle$, where the angle brackets imply thermodynamic averages. Furthermore, that the thermodynamic grand potential is given by the minimum of $\Omega^{\mathrm{MF}}\left(T, \nu,\left\{c_{i}\right\}\right)$ with respect to arbitrary variations in the local concentration variables $\left\{c_{i}\right\}$

$$
\frac{\partial \Omega^{M F}}{\partial c_{i}}=0
$$

which leads to

$$
\left(\frac{\partial \Omega}{\partial c_{i}}\right)=k_{B} T \ln \frac{c_{i}}{1-c_{i}}+\left(\frac{\partial \Omega^{\mathrm{CPA}}}{\partial c_{i}}\right)-\nu_{i}=0
$$

whose solution defines the equilibrium set $\left\{c_{i}\right\}_{0}$ of concentrations.

The interpretation of $\Omega^{\mathrm{CPA}}\left(\left\{c_{i}\right\}\right)$ in eq. 36 is that it is the CPA grand potential corresponding to an inhomogeneous set of local concentrations $\{. \gamma\}$ i.e. the probability that a site, say $n$, is occupied by an $A$ atom, namely $c_{n}$, differs from site to site. We refer to this as the inhomogeneous CPA [18],[20]. The reason for the CPA electronic 
grand potential appearing in eq. 36 is that we are required by tne mean nesu theory to take averages with respect to the inhomogeneous product distribution

$$
P\left(\left\{\xi_{i}\right\}\right)=\prod_{i} p_{i}\left(\xi_{i}\right)
$$

where $p_{i}\left(\xi_{i}\right)$, which now varies from site to site, and is given by

$$
p_{i}\left(\xi_{i}\right)=c_{i} \xi_{i}+\left(1-c_{i}\right)\left(1-\xi_{i}\right) \text {. }
$$

As remarked earlier, it is for performing averages with respect to a probability function that is a product of independent site probability functions that the CPA was developed.

Although, this inhomogeneous KKR-CPA recipe implicit in eq. 36 can not be implemented numerically, it is a very useful formal device because it can be expanded about the homogeneous limit, i.e. $c_{i}=\bar{c} \forall i$, and it allows the calculation of the derivatives

$$
S_{i}^{(1)}=\left(\frac{\partial \Omega^{C P A}}{\partial c_{i}}\right)_{c_{i}=\varepsilon V_{i}}
$$

and

$$
S_{i j}^{(2)}=\left(\frac{\partial^{2} \Omega^{C P A}}{\partial c_{i} \partial c_{j}}\right)_{c_{i, c_{j}=\bar{c} V_{i}}}
$$

where $\bar{c}$ is the uniform concentration of the high temperature disordered phase.

The significance of $S^{(1)}$ is provided by eq. 38. As was pointed out by Gyöffry and $S t c=i s=[20]$ the second derivative is the mean-field approximation to, the OrsteinZernicke direct correlation function [43] and it plays a variety of important roles in the theory of compositional order. In particular $S_{i j}^{(2)}$ is related to the Warren-Cowley short-range order parameter, $\alpha_{i j}$,

$$
\alpha_{i j}=\frac{\left\langle\xi_{i} \xi_{j}\right\rangle-\left\langle\xi_{i}\right\rangle\left\langle\xi_{j}\right\rangle}{c_{i}\left(1-c_{i}\right)}
$$

that can be measured using $X$-ray, neutron and electron diffuse scattering experiments [44]. Specifically $\alpha(\overrightarrow{\mathbf{q}})$ is given by

$$
\alpha(\overrightarrow{\mathbf{q}})=\frac{1}{1-\beta \vec{c}(1-\bar{c}) S^{(2)}(\vec{q})}
$$

where $\alpha(\vec{q})$ and $S^{(2)}(\vec{q})$ are the lattice Fourier transforms of $\alpha_{i j}$ and $S_{i j}^{(2)}$ respectively.

The important point about eq. 44 is that $S^{(2)}(\vec{q})$ can be evaluated from information available at the end of a KKR-CPA calculation. For the case where only the band structure contribution to the grand potential is considered, the first term on the right of eq. 30, explicit expressions are given in reference [40].

Before showing the results of calculations of $S^{(2)}(\vec{q})$ for a number of alloy systems. a few preliminary comments are in order. Firstly, the configurational entropy that appears in eq. 36 is simply the ideal entropy of mixing. Thus, effects that that explicitly depend on higher order entropic contributions are neglected. However, by taking this tack we are able to retain the electronic interaction in there full generality. This allows us to identify the specific electronic mechanisms that are responsible for the particular form of the SRO of clustering seen in any particular system. Secondly, since the LDA-KKR-CPA method is a mean field theory of the effects of disorder on the electronic structure the overall theory has a high degree of internal consistency.

In what follows we shall begin by considering alloy systems were it is sufficient to approximate the electronic grand potential by the band structure contribution alone, 
i.e. systems were charge transfer effects contained in the double counting terms are small. This need not be an arbitrary approximation since the LDA-KKR-CPA charges associated with the various species are outputs of calculations. Subsequently, we shall comment on a system $\mathrm{Ni}_{c} \mathrm{Cr}_{1-c}$ were it is necessary to include these effects.

Finally, as regards subsequent calculations of transition temperatures for ordering and for phase separation the theory of the diffuse scattering intensity $\alpha(\overrightarrow{\mathbf{q}})$, as developed above, does not satisfy the conservation rule

$$
\alpha_{i i}=1 / \Omega_{B z} \int d q \alpha(q)=1
$$

This failure, results from the fact that MF.CF theory does not satisfy the fluctuationdissipation theorem. This generic failure of mean field theories has been known for a long time, together with a method, introduced by Onsager [45] and further developed by Brout and Thomas [46], of improving the mean field theory such that the fluctuationdissipation theorem is satisfied. The MF-CF theory can be similarly corrected [47], [48], [4.9] the resulting expression for $\alpha(\overrightarrow{\mathbf{q}})$ takes the form

$$
\alpha(\mathbf{q})=\frac{1}{1-\beta c(1-c)\left(S^{(2)}(\mathbf{q})-\Lambda_{c}\right)}
$$

where $\Lambda_{c}$ is a normalization constant that enforces the conservation rule 45 . In what follows, we shall refer to this variously, as the Onsager cavity field corrected theory or the mean spherical approximation. This correciion makes a significant improvement to phase transition temperature at little extra computational cost. Thus we will use it as a matter of course.

\subsubsection{Fermi-surface nesting}

The first application of the mean field concentration functional theory outlined above was to $\mathrm{Cu}$-rich $\mathrm{Cu}_{c} \mathrm{Pd}_{1-c}$ alloys [20]. These alloys are members of a large class of systems [50], [51] (and references therein) that either show concentration dependent diffuse scattering in the disordered phase or long period ordered structures (LPOS) or both. For the $\mathrm{Cu}_{c} \mathrm{Pd}_{1-c}$ Gyorffy and Stocks related the position and wavelength of the concentration dependent diffuse scattering peaks observed in electron diffraction experiments (see fig. 9) to the direction and length of spanning vectors connecting well defined, flat, parallel sheets of the alloy's Fermi surface. One of the major points about that work was that, even in disordered alloys, specific parts of the Fermi surface can survive alloying with the peaks in the Bloch Spectral function remaining sharp across the whole concentration range, and that these pieces of Fermi surface can then drive ordering phenomena. Furthermore, since in non-isoelectronic alloys, the Fermi surface dimensions are depend strongly on the concentration (electron to atom ratio) the ordering phenomena are also strongly concentration dependent. For $\mathrm{Cu}_{c} \mathbf{P d}_{1-c}$ alloys the particular part of the Fermi surface that gives rise to this Fermi surface nesting mechanism is normal to the $\Gamma$ - $K$ direction and can be clearly seen in fig.2. The specific nesting vector connects the flat pari of the Fermi surface in one Brillouin zone with its equivalent in an adjacent one.

Subsequent calculation of $S^{(2)}(\overrightarrow{\mathbf{q}})$ using the full KKR-CPA expressions gave the diffuse scattering patterns shown in fig. $9[20],[40],\{41]$. Also shown are the corresponding electron diffraction patterns taken from the work of Osisima and Watanabe that clearly show the four fold SRO diffuse scattering peaks splitting around the (110)-superlattice position. Furthermore, they show the rapid concentration dependence of this splitting. The large white areas in experimental diffraction patterns are the Bragg peaks associated with the underlying fcc symmetry of the disordered phase. 

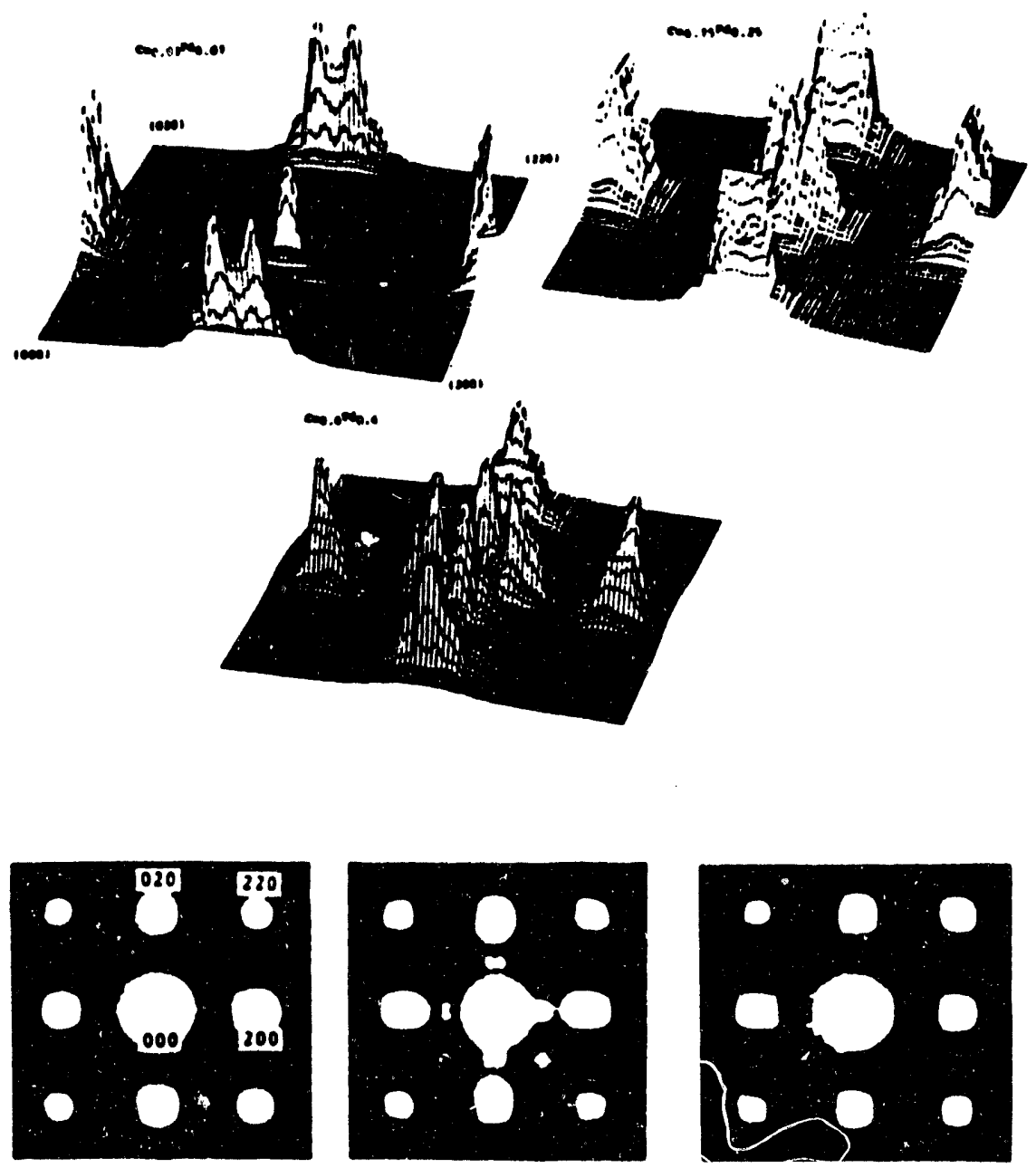

Figure 9: Calculated Warren-Cowley SRO parameter $\alpha(\vec{q})$ in the plane containing the reciprocal lattice points $000,020,022$, and 002 for various $\mathrm{Cu}_{c} \mathrm{Pd}_{1-c}$ alloys (upper frames) and corresponding Electron diffraction patterns taken from the work of Oshima and Watanabe. 
At low temperature $\mathrm{Cu}$-rich $\mathrm{Cu}_{c} \mathrm{Pd}_{1-c}$ alloys order into a series of one and inv dimensional LPOS [50],[51]. Recently, Ceder et. a! [51] have used the calculated MFCF values of $S^{(2)}(\vec{q})$ to calculate the phase diagram of the LPOS. In this work they make the assumption that $S^{(2)}(\vec{q})$ can be interpreted as a Fourier trans Jrmed pair potential, $v(\vec{q})$, and then use it in a mean field free energy expression to calculate the range of stability of the LPOS. Their results are summarized in fig. 10 and in table 1 $[20],[40],[41]$. The notation for the LPOS is that of Fisher and Selke [52]. As can be
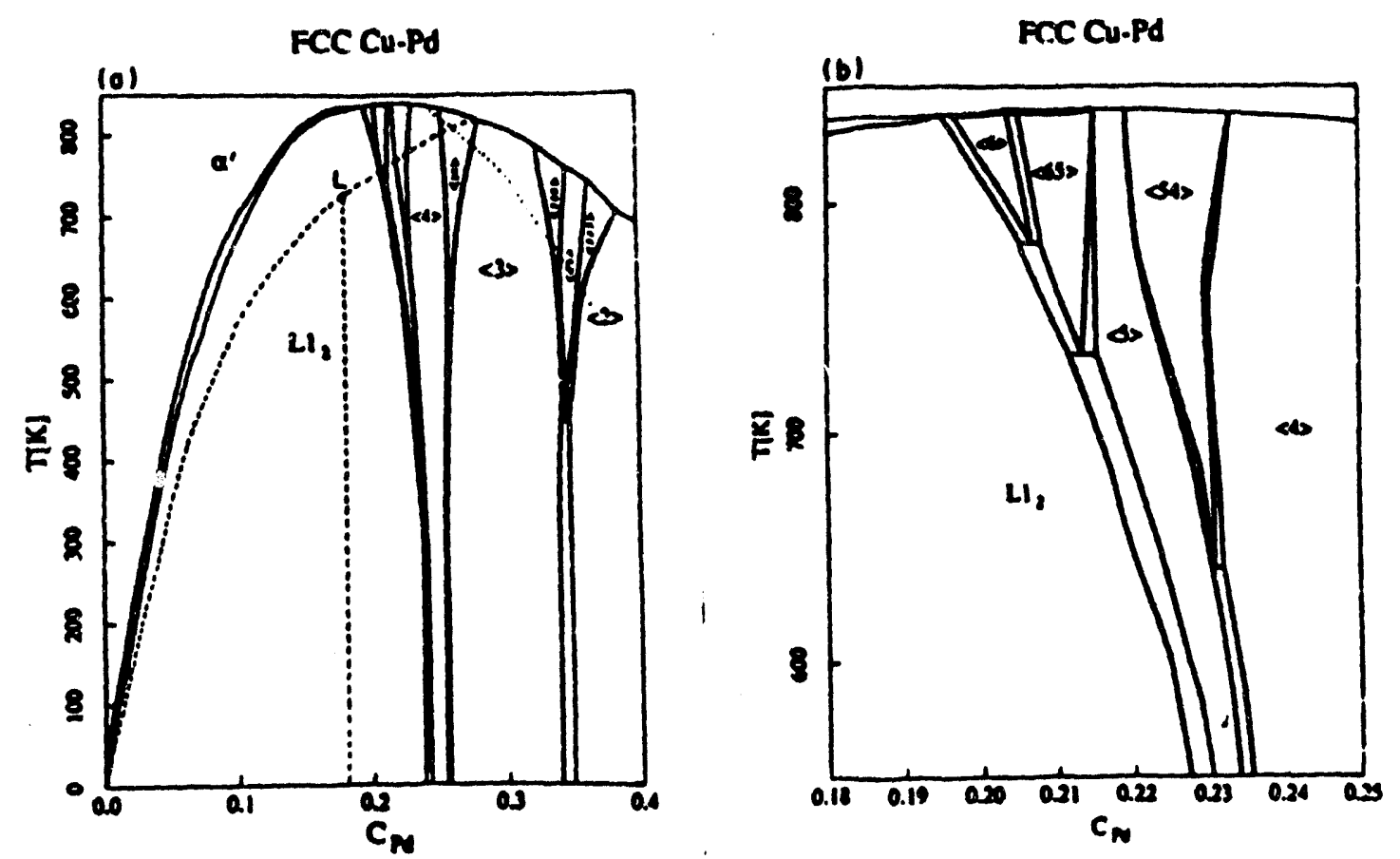

Figure 10: Left frame: Phase diagram as calculated within the Bragg-Williams approximation using the LDA-KKR-CPA effective interactions. Phases with modulation period larger than 4 are not labeled because their region of stability is small. Right frame: Enlargement of the $\mathrm{Cu}$-rich region showing the LPOS with longer periods.

seen from table 1 there is excellent agreement between the list of structure that are calculated to exist and those that have actually been observed.

\subsubsection{Concentration Dependent Interactions}

As we saw in the previous subsection, the rapid concentration dependence of $\alpha(\vec{q})$ for $\mathrm{Cu}$-rich $\mathrm{Cu}_{c} \mathrm{Pd}_{1-c}$ alloys implies that the underlying interactions are very concentration dependent. However, Fermi surface nesting is not the only way such extreme concentration dependence can arise.

As we remarked earlier $2.3 \mathrm{Ag}_{c} \mathrm{Al}_{1-c}$ is a particularly interesting alloy system in that the calculations of the energies of mixing, $\Delta E^{\mathrm{mix}}$, are positive for Al-rich alloy suggesting that these alloys want to phase separate, and negative for Ag-rich alloy suggesting that these alloys want to order at low temperature. This conjecture is supported by explicit calculation of $\alpha(\vec{q})$. In fig. 11 we show calculated $S 20$ diffuse scattering maps for $\mathrm{Ag}_{0.2} \mathrm{Al}_{0.8}$ and $\mathrm{Ag}_{0.8} \mathrm{Al}_{0.2}$. Clearly, for the $\mathrm{Al}$-rich alloy the peaks in $\alpha(\vec{q})$ are at the fundamental Brags positions commensurate with the alloy exhibiting a tendency to phase separate at low temperature. For the Ag-rich alloy the peaks in $\alpha(\overrightarrow{\mathbf{q}})$ are at $(1 / 2,1 / 2,1 / 2)$ positions, the super-lattice positions corresponding to the $\mathrm{CuPt}$-structure, indicative that these alloys would show this type of ordering tendency 

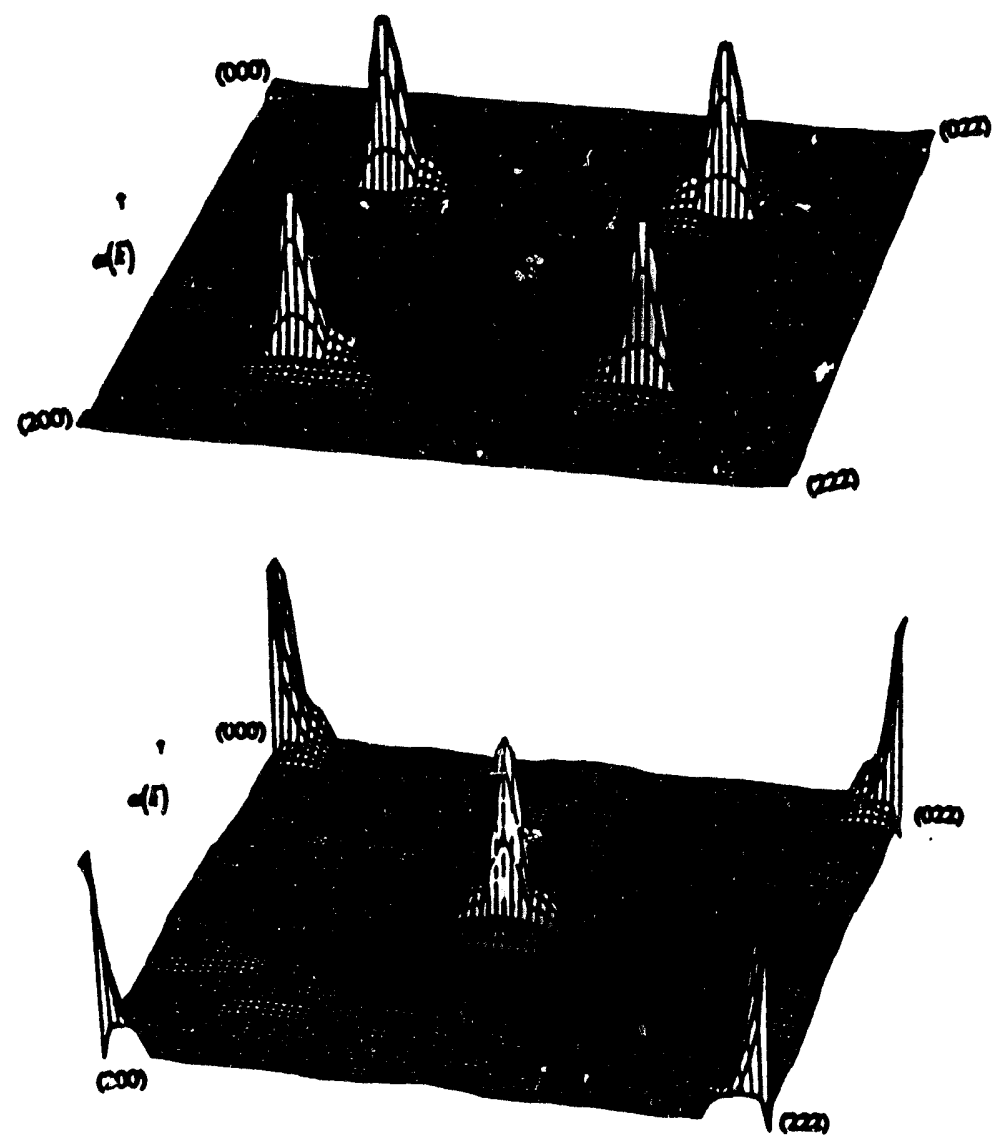

Figure 11: Calculated Warren-Cowley SRO parameter $\alpha(\vec{q})$ for $A_{\text {go.8 }} A_{0.2}$ (upper) and Ago.2A 0.8 (lower) alloys. The plot is for the plane containing the reciprocal lattice points $000,200,022$, and 222 . The calculations are for a temperature that is just above the miscibility gap. 


\begin{tabular}{|c|c|c|}
\hline Phase & $\begin{array}{l}\text { Cakculated } \\
\text { stability }\end{array}$ & $\begin{array}{l}\text { Experimentally } \\
\text { observed? }\end{array}$ \\
\hline $\begin{array}{c}\dot{0} \\
\dot{L}, \\
\langle 9\rangle \\
\langle 8\rangle \\
\langle 1\rangle \\
\langle 6\rangle \\
\langle 65\rangle \\
\langle 3\rangle \\
\langle 34\rangle \\
\langle 4\rangle \\
\langle 4\rangle \\
\langle 43\rangle \\
\langle 3\rangle \\
\langle 332\rangle \\
\langle 32\rangle \\
\langle 322\rangle \\
\langle 2\rangle \\
\langle 1\rangle \\
\langle 21\rangle\end{array}$ & $\begin{array}{l}\mathbf{Y} \\
\mathbf{Y} \\
\mathbf{N} \\
\mathbf{N} \\
\mathbf{N} \\
\mathbf{Y} \\
\mathbf{Y} \\
\mathbf{Y} \\
\mathbf{Y} \\
\mathbf{Y} \\
\mathbf{Y} \\
\mathbf{Y} \\
\mathbf{Y} \\
\mathbf{Y} \\
\mathbf{Y} \\
\mathbf{Y} \\
\mathbf{N} \\
\mathbf{N}\end{array}$ & 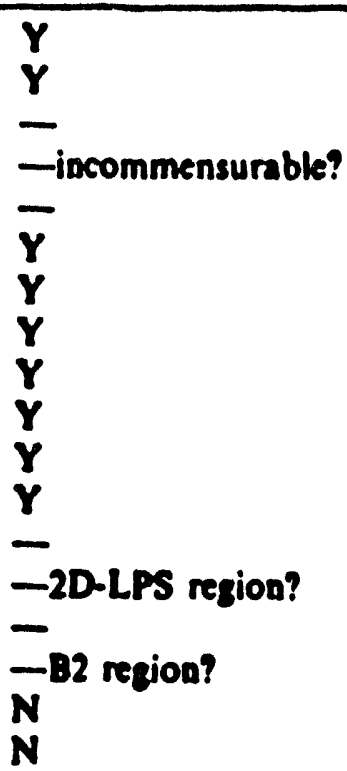 \\
\hline
\end{tabular}

Table 1: Calculated and observed LPOS in $\mathrm{Cu}_{c} \mathrm{Pd}_{1-c}$ alloys. The structures that whose stability was analyzed are listed in column 1 . Column $2(3)$ indicates whether the structure is found to be stable in the calculations (experiments)

at low temperature. It is very satisfying that the results of calculations of $S^{(2)}(\vec{q})$ are bear out what was expected on the basis of the energy calculations.

The experimental phase diagram is quite complex. For Ag-rich alloys phases that are based on kcc and hcp lattices dip down to low temperatures. These are phases that we have not considered since our calculations were performed for an underlying lattice that is fcc. Thus, the sxistence of the fcc based CuPt structure is masked. For Al-rich alloys the siturtion is more interesting. The experimental equilibrium phase diagram shows, at room temperature, a two phase field between an fcc $\alpha$-phase disordered solid solution of $\mathrm{Ag}$ in $\mathrm{Al}$ and a hcp related phase, based on the chemical composition $\mathrm{Ag}_{2} \mathrm{Al}$, which is the equilibrium phase for $\mathrm{Ag}$ content $>\mathbf{4 0}$ atomic percent [33], [53]. However, it is known [53] that within the two phase field that there is a metastable miscibility gap and that this is responsible for driving the formation of Guinier-Preston(GP) zones [54] [55], [56]. GP-zones provide a fundamental strengthening mechanism in many commercial Al-based alloys, for exar.jple alloys based on Al with a few percent $\mathrm{Cu}$ find, amongst a wide set of uses, application in aircraft skins. In $\mathrm{AlAg}$ alloys, the GP-zones are coherent precipitates that form under appropriate annealing conditions and are comprised of essentially pure Ag even though the parent phase is very $\mathrm{Al}$ rich [53]. Presumably, the miscibility gap found in our calculations for fcc phase is the one that is responsible for providing the driving mechanism for the formation of the GP-zones.

\subsubsection{Band Filling}

There are fairly general arguments based on the tight binding model that suggest alloys between late transition metals with roughly half filled d-bands should order whilst those with nearly empty or nearly full d-bands should cluster in the disordered phase and, 
therefore, pisase separate at low temperatures $[57,58,59,60,61]$. The exact positions of the crossover from clustering to SROO depends on the details of the Hamiltonian, but the trend is roblist. Further, experimental binary alloy phase diagrams involving transition metals from the same series show ample evidence of this general trend [33].

The $\mathrm{Pd}_{\mathrm{c}} \mathrm{Rh}_{(1-c)}$ alloy system is a particularly good example of this band filling effect. These late 4d-transition metals are of almost equal size, and there are only small strain and charge transfer effects. In such a system it is expected that the eigenvalue sum shouid dominate the energetics and the clustering predicted by tight binding models should not be obscured by some other mechanism. The phase diagram of $\mathrm{Pd}_{c} \mathrm{Rh}_{(1-c)}$ is very simple, below melting it is a solid solution until the iemperature drops into the miscibility gap when it phase separates into Pd-rich and $\mathrm{Rh}$-rich phases.

The CPA energy of mixirg is positive indicating that $\mathrm{Pd}$ and $\mathrm{Rh}$ would prefer not to mix but to form clusters. Figure 12 shows the calculated short range order diffuse scattering pattern obtained on the basis eq. 44. Clearly, the peaks in the diffuse

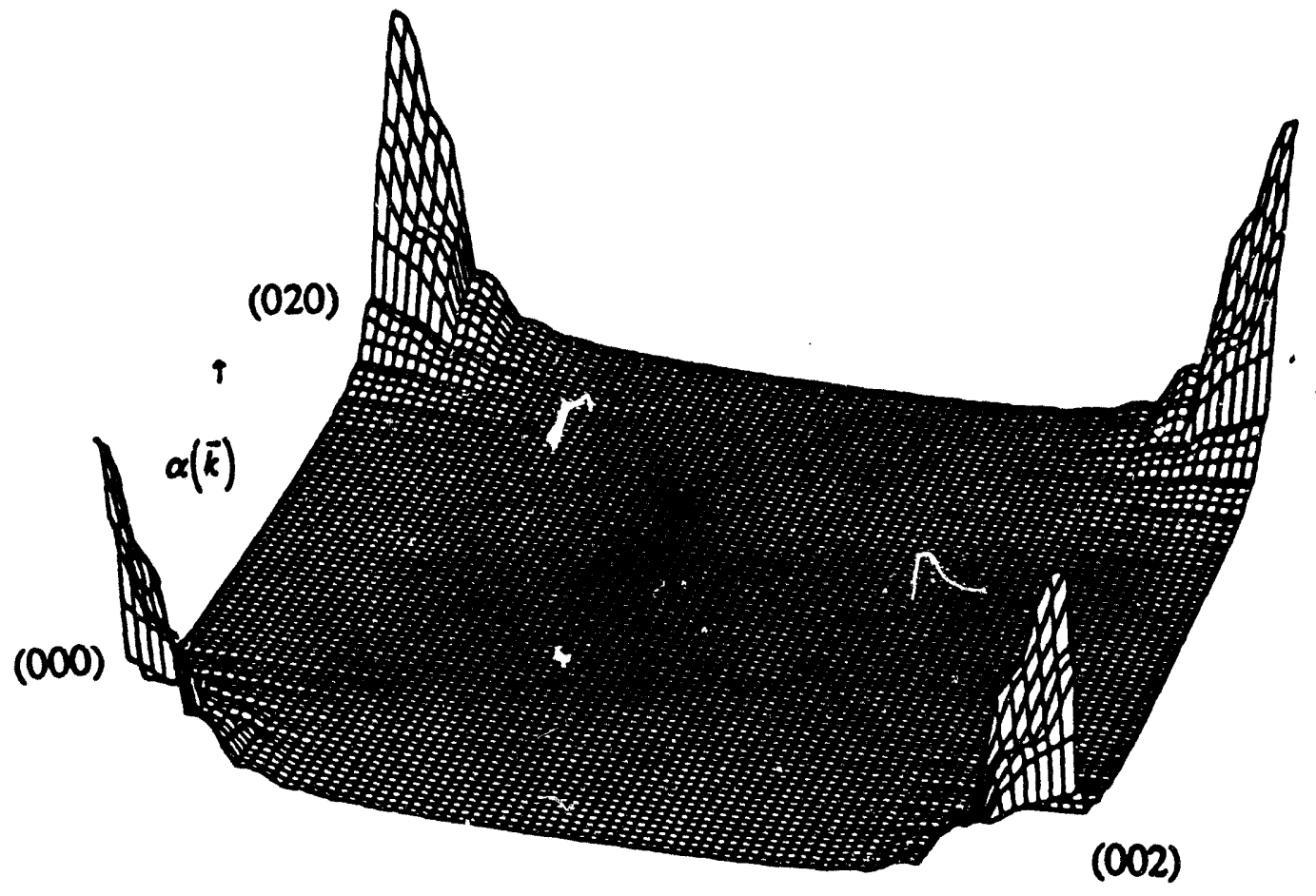

Figure 12: Calculated Warren-Cowley SRO parameter $\alpha(\vec{q})$ for $P d_{0.25} R h_{0.75}$. The plot is for the plane containing the reciprocal lattice points 000, 020, 022, and 002 . The calculations are for a temperature that is just above the miscibility gap.

scattering are at the reciprocal lattice positions of the underlying fec lattice, indicative of phase separation and consistent with the energies of mixing.

In this lecture we bypass concerns about detailed agreement with experiment and concentrate on the way in which clustering arises in the CF theory and its relation to band filling. To this end we specialize the CF expression for the diffuse intensity at $\overrightarrow{\mathbf{q}}=\mathbf{0}$ which indicates the strength of clustering. If we neglect matrix elements and approximate the energy integral in the full expression for $S^{(2)}(\vec{q}, T)$ by the contribution from the first Matsubara pole $\omega_{1}=\pi k_{B} T$ which, for $\mathrm{Pd}_{c} \mathrm{Rh}_{1-c}$ is the dominant contribution, then

$$
S^{(2)}(\overrightarrow{\mathbf{q}}=0, T) \approx \int d \overrightarrow{\mathrm{k}} \Im G^{C P A}\left(\overrightarrow{\mathbf{k}}, \epsilon_{F}+\omega_{1}\right) \Re G^{C P A}\left(\overrightarrow{\mathrm{k}}, \epsilon_{F}+\omega_{1}\right)
$$


where $G^{C P A}(\overrightarrow{\mathbf{q}}, z)$ is the lattice Fourier transform of the CPA Green lunctuun at wic complex energy $z=\epsilon_{F}+w_{i}$, the imaginary part of which is the Bloch spectral function $A(\vec{q}, \epsilon)$ defined in eq.23. Even though we evaluate $S^{(2)}(\vec{q}, T)$ using the full KKR-CPA formula [40] the approximate form of eq. 47 it instructive because it relates the diffuse scattering to the spectral function, features of which, as we have pointed out earlier, are measurable using photoelectron spectroscopies and determine such properties as the residual resistivity. Thus a link is made between physics related measurements of the underlying electronic structure and the metallurgical interesting phase stability of the system.

Using the relation $d G / d \epsilon=-G G$, eq. 47 can be written in the form

$$
S^{(2)}(\vec{k}=0, T) \approx \int_{\Omega_{B 2}} d \vec{q} A^{\prime}
$$

where

$$
A^{\prime}=-\frac{d \Im G\left(\vec{q}, \epsilon_{F}+\omega_{1}\right)}{d \epsilon}
$$

The Bloch spectral function $A(\vec{k} ; \epsilon)$ for $\mathrm{Pd}_{c} \mathrm{Rh}_{1-c}$ at $\mathrm{c}=0.5$ is plotted in fig. 13 along with the integrand occurring in eq. 48. $A(\overrightarrow{\mathbf{k}} ; \epsilon)$ is also shown for the direction $\Gamma-X$ as a function of energy for energies above the Fermi level. The inset shows the bands of pure $\mathrm{Pd}$ along this direction [62]. Note, just above $\epsilon_{F}$, the uppermost $d$-band flattens out to meet the zone boundary. In the disordered alloy, the large negative contributions seen in $A^{\prime}$ occur because $\epsilon_{F}$ is within $\gamma$ of the intercept of the uppermost $d$-band with the zone boundary, where $\gamma$ is the energy half width of $A\left(\vec{k} ; \epsilon_{F}\right)$.

A schematic depiction of how this occurs is shown in fig. 14. For the case of a band (top-frame) where $\gamma$ is small (right frames) $A\left(\vec{k} ; \epsilon_{F}\right)$ is $\delta$-function like and is contained within the $\mathrm{BZ}$ (middle-frame) consequently the integral over the $\mathrm{BZ}$ of its derivative (lower-frame) is zero. For a band where $\gamma$ is large on the scale of the dispersion (left frames) $A\left(\vec{k}_{;} \epsilon_{F}\right)$ may be asymmetrical and intersect with the $\mathrm{BZ}$ boundary. In this case the integral over the $\mathrm{BZ}$ of its derivative is large giving rise to large (clustering) contributions to $S^{(2)}(\vec{q}, T)$. Clearly, as can be seen in fig. 13 this is the case for $\mathbf{P d}_{c} \mathbf{R h}_{1-c}$. Quite generally, we can assert that large clustering contributions to $S^{(2)}(\overrightarrow{\mathbf{q}}, T)$ will appear when $\epsilon_{F}$ is just below an extremum of a band for an appreciable part of the BZ. Obviously, this situation occurs most readily when $\epsilon_{F}$ is close to the top of the $d$-band.

At high temperatures $S^{(2)}(\vec{k}=0, T)$ goes to zero and the system is random as dictated by entropy. However, as the temperature is reduced $S^{(2)}(\vec{k}=0, T)$ grows and clustering type SRO becomes stronger. Eventually, the denominator in eq. 44 will go to zero indicating that the system is unstable to phase separation; infinitesimal fluctuations in concentration reduce the free energy. This is the spinodal temperature, T. . Atove the spinodal $\alpha(\vec{q})$ is peaked at the $\Gamma$-point as in fig. 12 and this peak grows as $T$ is reduced, becoming singular at $T_{\text {. }}$. The concentration dependence of $T_{0}$ is shown in fig 15 along with the immiscibility phase boundary. The phase boundary was obtained by applying the common tangent construction to the calculated free energy. The free energy of the solid solution as a function of concentration was found by making a polynomial fit in $c$ to $S^{(2)}(\vec{k}=0, T)$ and integrating twice over $c$. The calculated phase boundary is in reasonable accord with experiment [33] although the calculated transition temperatures are greater than experiment by about $200 \mathrm{~K}$.

\subsubsection{Band Filling, Off-diagonal Randomness and Relativity}

One of the strengths of first principles approaches to studying phase stability is that they have the possibility to allow us to understand ordering/clustering in alloy systems 


\section{FERMI SURFACE CONTRIBUTIONS TO ! FOR Pd0.5Rh0.5}

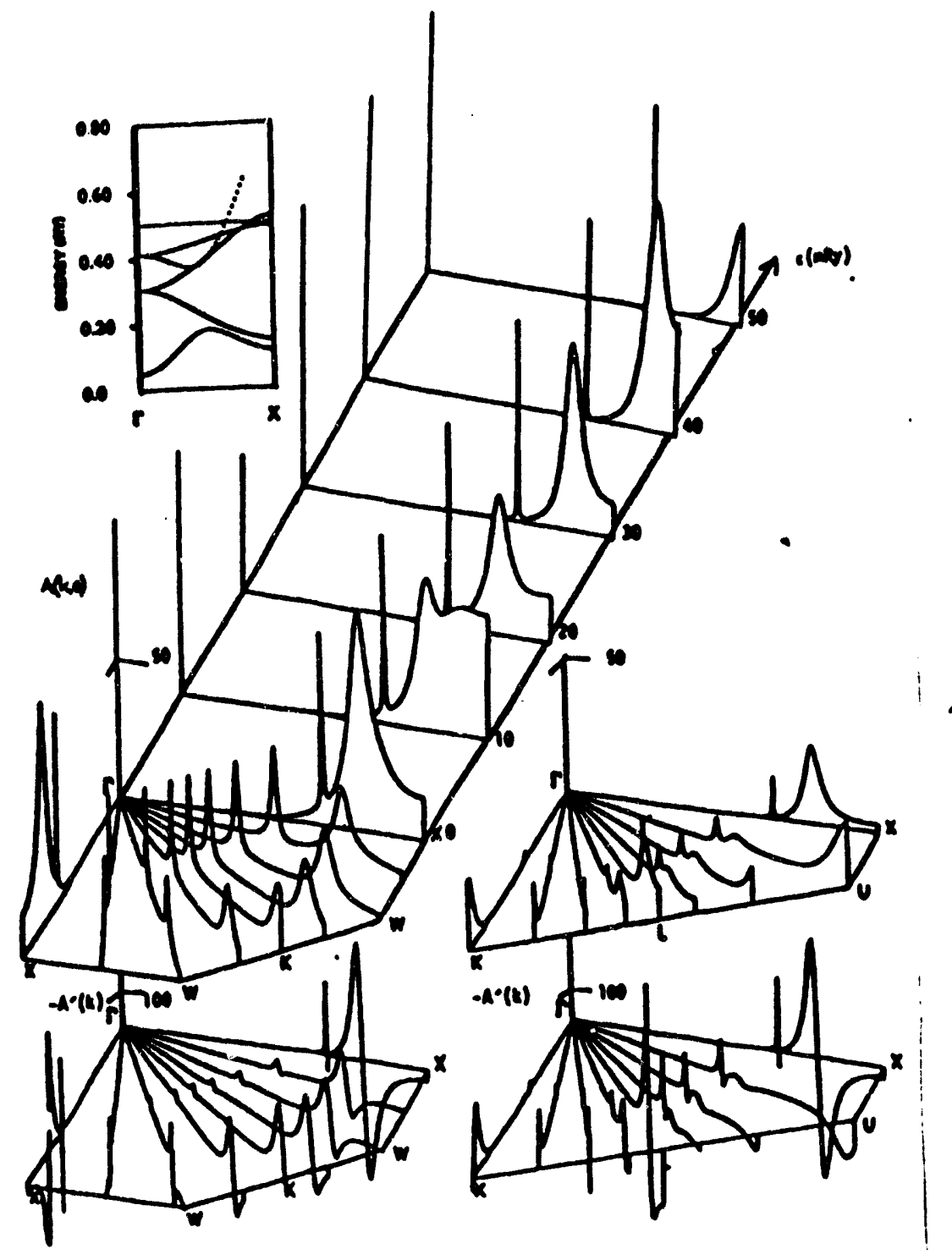

Figure 13: Calculated $A\left(\vec{k}_{;} \epsilon\right)$ for $\mathrm{Pd}_{0.5} \mathrm{Rh}_{0.5}$ alloys at the Fermi energy for various direction in the $\mathrm{BZ}$ and for the $\Gamma-X$ direction for various energies above $\epsilon_{F}$. The lower plots show the $\overrightarrow{\mathrm{k}}$ dependence of the $A^{\prime}$ the dominant contribution to $S^{(2)}(\overrightarrow{\mathrm{q}}, T)$ 


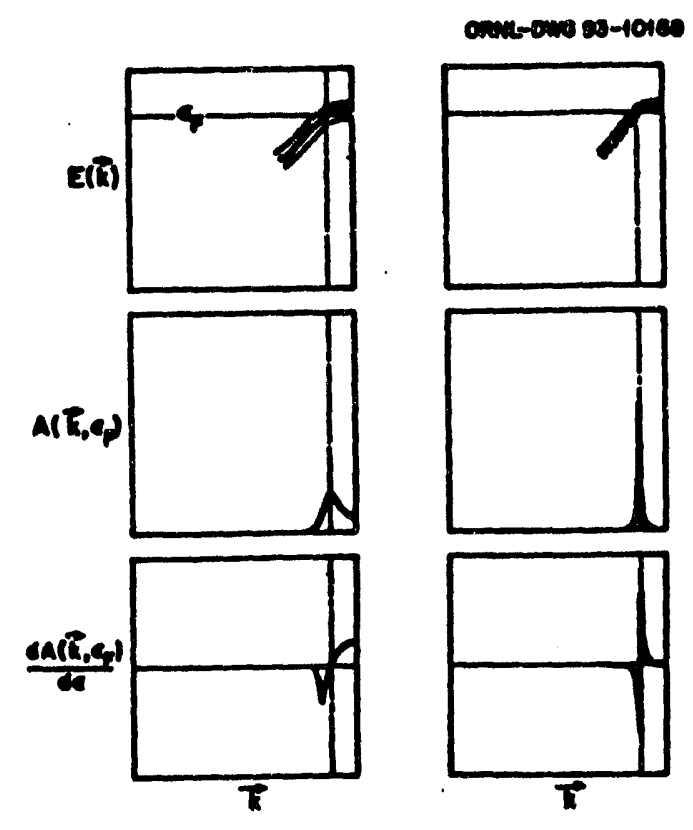

Figure 14: Schematic of alloy bands (upper), $A\left(\vec{k} ; \epsilon_{F}\right)$ (middle), and $A^{\prime}$ (lower) for the case of an alloy band structures that are sharp (right) and broad (left) on the scale of the dispersion

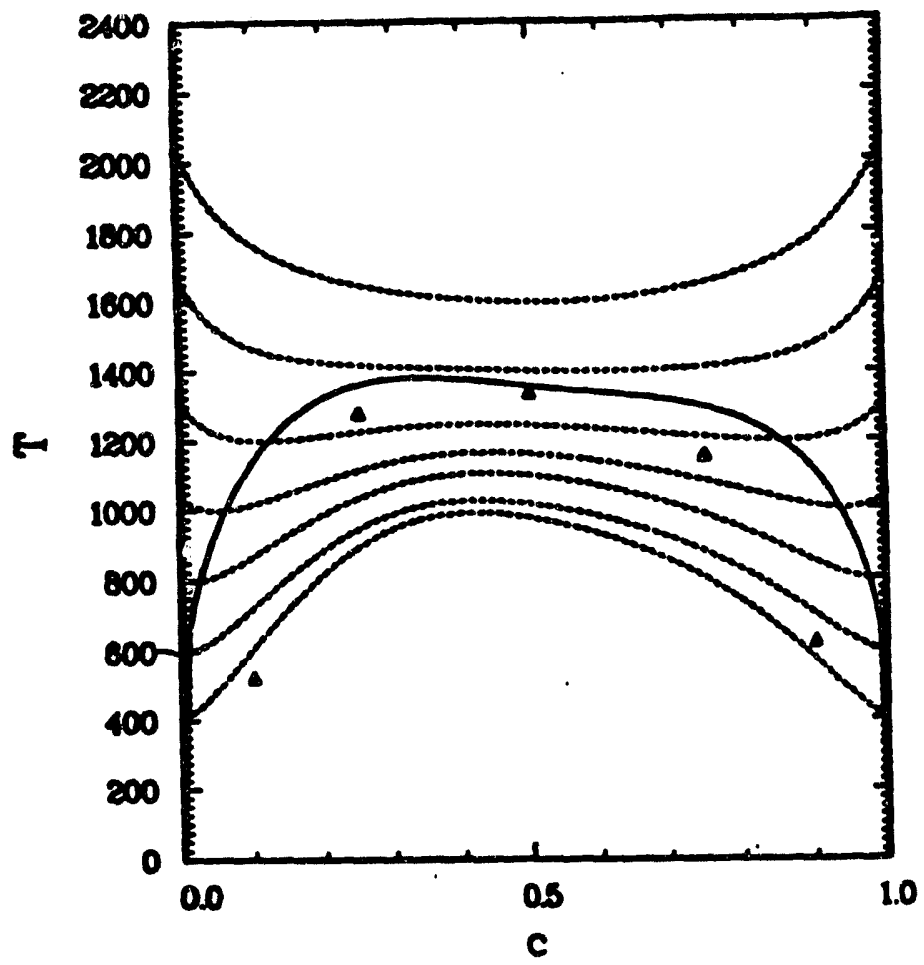

Figure 15: Calcuiated phase boundary (solid line) and spinodal temperatures (triangles) and free energies at various temperatures (dashed lines) for $\mathbf{P d}_{c} \mathbf{R h}_{1-c}$ alloys 
where different underlying mechanisms are competing. I he Nirt alloy system provucs a nice example of this where the competition is between band filling, off-diagonal randomness and relativity.

Despite the fact that the $\mathrm{Ni}_{c} \mathrm{Pt}_{1-c}$ alloy system is comprised of two elemental metals from the end of there respective transition metal series ( $3 d$ for $\mathrm{Ni}$ and $5 d$ for $\mathrm{Pt}$ ) these alloys exhibit SRO in the disordered phase [63] and form ordered structures $\left(\mathrm{Ll}_{2}, \mathrm{Ll}_{0}\right)$ upon slow cooling to low temperatures [33] rather than clustering and phase separations as would be expected on the basis of band filling arguments. Aware of this exception to the rule, Treglia and Ducastelle [64] studied $\mathrm{Ni}_{c} \mathrm{Pt}_{1-c}$ using a tight-binding model Hamiltonian and concluded that there was no simple way of avoiding the prediction that $\mathrm{Ni}_{c} \mathrm{Pt}_{(1-c)}$ should cluster in the disordered phase and phase separate at low temperatures. In the end, they suggested that spin-orbit coupling, which was neglected in their non-relativisiiic treatment of the problem, might give rise to a repulsion between the $\mathrm{Pt}$ atoms and hence override the usual band filling argument. To substantiate or reject this interesting hypothesis was the purpose of two recent reconsiderations of the problem by Pinski et al. [65] and Lu et a. [17], [66]. Unfortunately, these calculations resulted in apparently conflicting results. The first suggested that the ordering tendency in the $\mathrm{Ni}_{c} \mathrm{Pt}_{(1-c)}$ system is due to a size effect that results from the very different $d$-band widths of $\mathrm{Ni}$ and $\mathrm{Pt}$ (off-diagonal randomness in the language of tight binding models), whilst Lu et al. [17] concluded that a relativistic effect but not specifically to do with the spin-orbit coupling was responsible.

In fig. 16 we show the calculated Warren-Cowley short-ranged order parameter

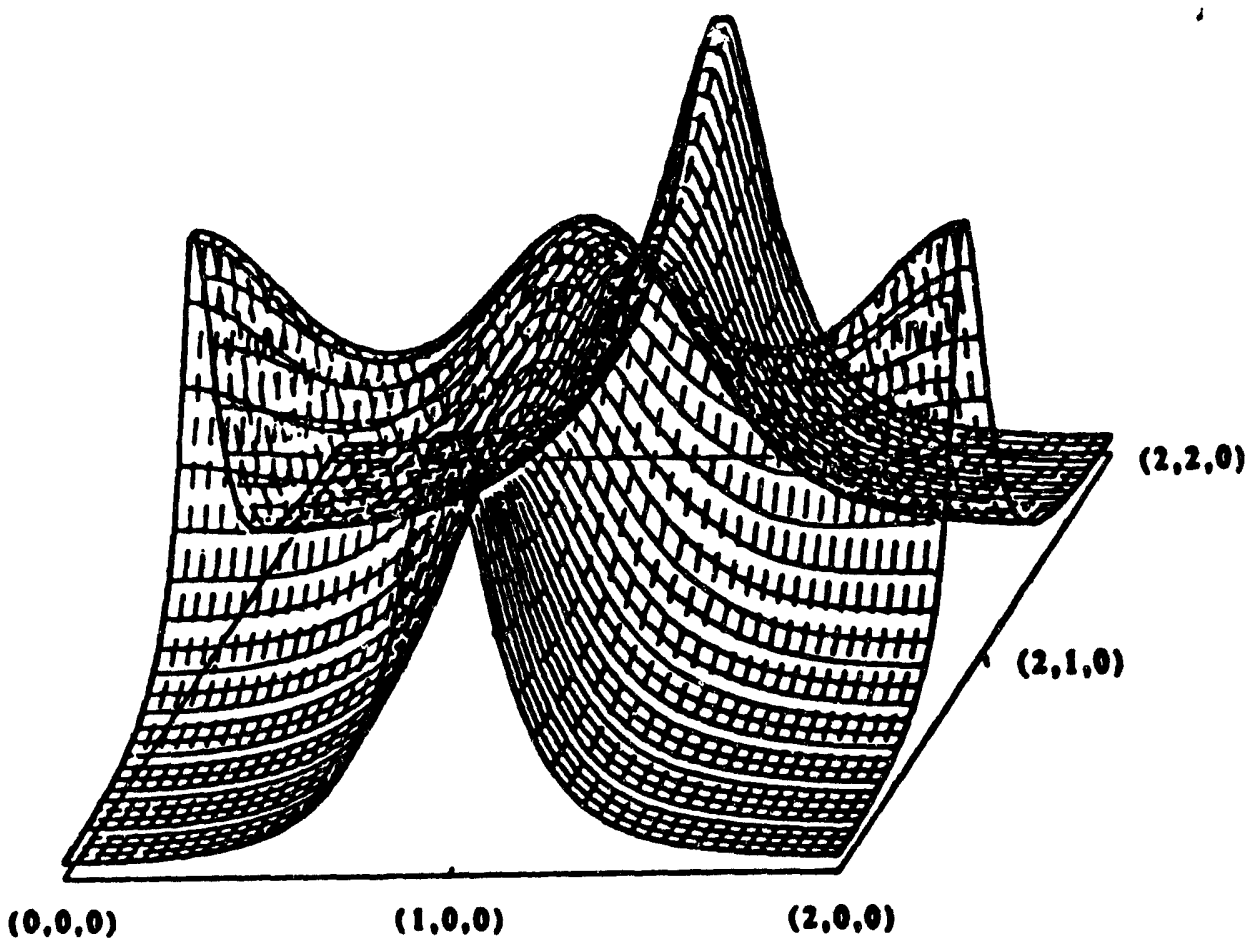

Figure 16: $\alpha(\vec{q})$ in the $q_{z}=0$ plane for $\mathrm{Ni}_{0.5} \mathrm{Pt}_{0.5}$ for a temperature about $10 \%$ greater than the theoretical ordering temperature. The peaks are at the X-points of the FCC Brillouin zone, indicating the tendency for the alloy to order along the (100) direction, i.e., into the $\mathrm{Ll}_{0}$ structure at low temperatures [65]

$\alpha(q)$ for $\mathrm{Ni}_{0.5} \mathrm{Pt}_{0.5}$ obtained by Pinski et. al. [65]. $\alpha(q)$ peaks at the $X$-point consistent with the $\mathrm{Ll}_{0}$ low temperature ordered phase. These calculations are based on a entirely non-relativistic treatment of the electronic structure. Thus, Pinski et. al. conclude that relativity was not responsible for driving the ordering, rather, it was the off- 
diagonal randomness that is a consequence of the size difference between $\Lambda_{1}$ and $r_{L}$ that suppresses the band filling mechanism.

Lu et al. [17], [66] calculated the formation energy

$$
\Delta E\left(V_{L_{1}}, V_{N i}, V_{P t}\right)=E\left(N i P_{t}, V_{L 1_{0}}\right)-\frac{1}{2}\left[E\left(N i, V_{N_{i}}\right)+E\left(P t, V_{P_{t}}\right)\right]
$$

where $V_{\alpha}$ is the equilibrium volume of the system indicated by the suffix $\alpha=L 1_{0}$, $\mathrm{Ni}$ and $\mathrm{Pt}$ respectively. The calculations were performed using state-of-the-art selfconsistent, scalar-relativistic and non-relativistic FLAPW calculations [17], [66]. They found that non-relativistically $\Delta E$ is positive implying phase separation at low temperatures, but scalar-relativistically it is negative and, therefore, consistent with the observed ordering tendency. They therefore concluded that relativity, specifically the mass-velocity and Darwin terms (since they also neglected spin-orbit coupling) was responsible for the ordering tendency.

Subsequent to the original work, there have been further corr ments from both sides of this debate [67] [68]. Györffy et. al. [69] have suggested a possible resolution of these apparently conflicting results. Here we will not recount this debate, rather, we will sum up the essence of the resolution offered by Gyorffy et. al.. Firstly, relativity is a necessary component in the resolution since it has the effect of reducing the size of the $\mathrm{Pt}$ atom. This results from the inclusion of the mass velocity and Darwin terms in the scalar relativistic calculations, which has the effect of pulling down the energy of the $6 s$-states relative to the $5 d$-states and making them more compact. Gyorffy et. al. argue that without this effect the size difference between (non-relativistic) $\mathrm{Pt}$ and $\mathrm{Ni}$ would be so large as to induce strain fluctuations (see below) that would favor phase separation. Thus, the effect of relativity is to reduce the effective size of the $\mathrm{Pt}$ atoms, thereby allowing $\mathrm{Ni}$ and $\mathrm{Pt}$ to mix. However, once the $\mathrm{Pt}$ and $\mathrm{Ni}$ atoms are of sufficiently similar size to allow mixing at high temperature, as they are in the scalar relativistic calculation, the mechanism identified by Pinskj et. al., namely the widely differing $d$-band widths of $\mathrm{Pt}$ and $\mathrm{Ni}$, would then drive the ordering.

This latter conjecture is based on the observation that the $d$-band structure in the density of states of $\mathrm{Ni}_{c} \mathrm{Pt}_{1-c}$ alloys is largely unaffected by the inclusion of the relativistic effects, even spin-orbit coupling. Since, it is this structure that is responsible for the driving mechanism identified by Pinski et. al. the inclusion of relativity does not suppress this mechanism. To illustrate this point, we show LDA-KKR-CPA calculations of the densities of states of disordered $\mathrm{Ni}_{0.5} \mathrm{Pt}_{0.5}$ alloys calculated both nonrelativistically and fully relativistically. The latter calculations were performed using a version of the LDA-KKR-CPA method described earlier that is based on solving the Dirac equation rather than the Schrödinger equation [70].

\subsubsection{Charge Transfer Effects}

So far we have ignored all contributions to $S^{(2)}(\overrightarrow{\mathbf{q}})$ save those resulting from the band structure contribution to the grand potential. In systems that exhibit significant charge transfer this is not sufficient. Recently, the basic MF-CF theory of Gyorffy and Stocks [20] has been extended by Johnson, Staunton, and Pinski [48] to include the neglected double counting contributions, namely, those from the electrostatic and exchange-correlation energy.

One may broadly speak of the electrostatic contributions to $S^{(2)}(q)$ in terms of 'charge transfer', $\Delta Q=\left(Q_{A}-Z_{A}\right)-\left(Q_{B}-Z_{B}\right)$, where $\Delta Q$ is the net gain of charge if a site in the homogeneous alloy is occupied by an $A$ atom or the net loss if a $B$ atom is placed at that site. This can be a 1 gge contribution and it describes the 


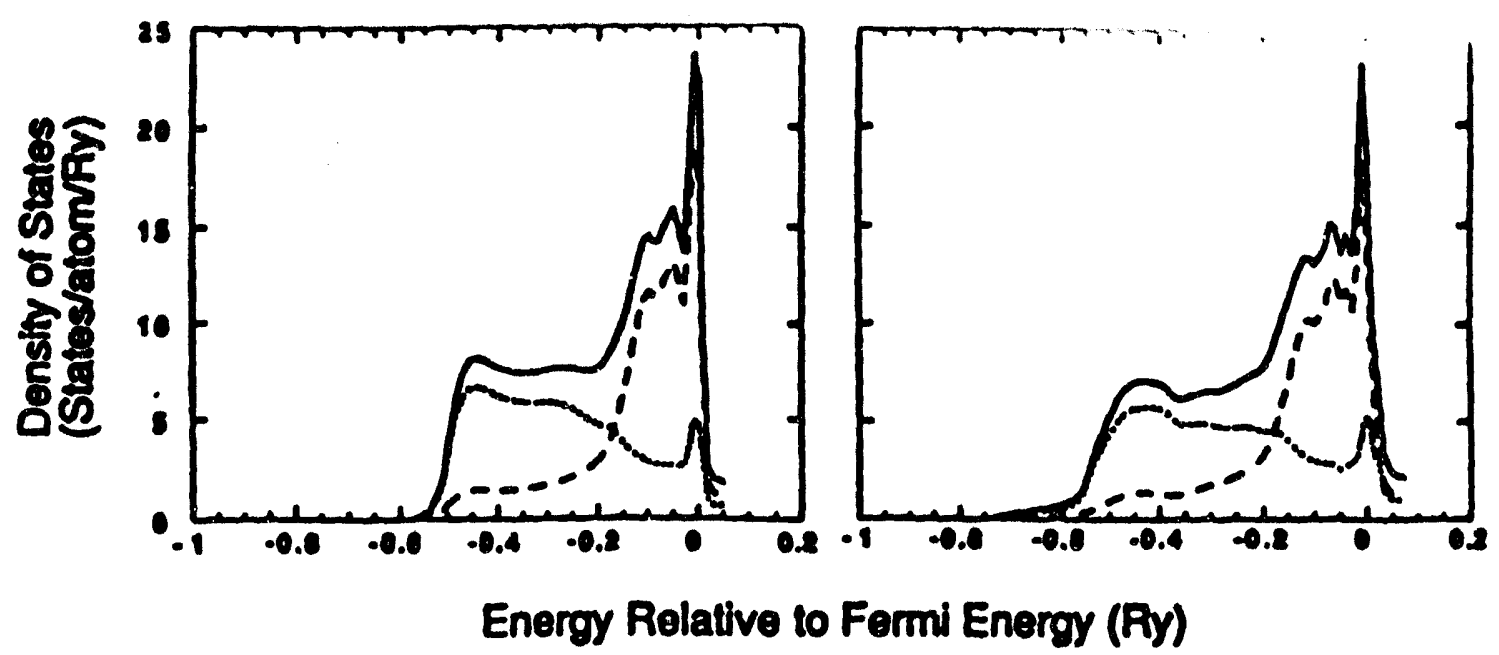

Figure 17: Non-relativistic (left) and relativistic (right) SCF-KKR-CPA densities of states (DOS) for $\mathrm{Ni}_{0.5} \mathrm{Pt}_{0.5}$. Solid line: total DOS. Dashed line: Ni-contribution. Dotted line: Pt-contribution. Charge self consistency reduces the effects of the massvelocity and Darwin terms, and the relativistic and non-relativistic DOS's look surprisingly similar, confirming, indirectly, the validity of the non-relativistic approach to NiPt.

electrostatic interaction between these equal and opposite charges and, in wave-vector space, is largest for those values of $q$ which are compatible with a simple $A-B$ ordering concentration wave.

The charge rearrangement terms result roughly from how the electrostatic interaction is screened through an adjustment of the occupation of the electronic states. So, if the averaged density of states at the Fermi energy, $\bar{n}\left(\varepsilon_{F}\right)$, is large, charge screening effects are important. If the difference of the averaged densities of states associated with $A$ and $B$ sites i.e. $\Delta n\left(\varepsilon_{F}\right)=\left|n_{A}\left(\varepsilon_{F}\right)-n_{B}\left(\varepsilon_{F}\right)\right|$ is sizable then screening results in the re-occupation of electronic states associated with one type of site rather than the other.

For those alloys characterized by both small effective 'charge transfers' $\Delta Q$ and small densities of states at the Fermi level, $n_{A}\left(\varepsilon_{F}\right)$ and $n_{B}\left(\varepsilon_{F}\right)$, the compositional correlations are dominated by the band-filling term $S_{\text {bend }}^{2}(q)$, such is the case for $C u P d$ alloys where SRO is determined by Fermi surface nesting [40]. In systems which also have small effective charge transfers, $\Delta Q \approx 0$, but differ in that they possess both a sizable $\bar{n}\left(\varepsilon_{F}\right)$ and $\Delta n\left(\varepsilon_{F}\right)$, it turns out that the charge arrangement is still sensitive to the compositional environment and there is an important contribution to the interchange energy $S^{(2)}(q)$ from charge re-arrangement and screening contributions. This can be considered loosely as an effect coming from 'local' Fermi energy adjustments as the number of electronic states available varies with atomic composition.

For alloys in which the atomic species have differing electronegativities and therefore non-negligible $\Delta Q$, once again we find that this category can be further sub-divided according to the nature of the electronic structure in the vicinity of the Fermi energy. For systems with large $\Delta Q$ but small $\Delta n\left(\varepsilon_{F}\right)$, the dominant functional form in these cases is roughly a sum of the band-filling energy $S_{\text {band }}^{2}(q)$ and a screened electrostatic interaction between charges $+\Delta Q$ and $-\Delta Q$ with a screening length $l_{\text {acr }}$, i.e.

$$
S^{(2)}(\mathbf{q})=S^{\infty}(\mathbf{q})-\frac{\Delta Q^{2} C(\mathbf{q})}{\left(1+l_{\infty}^{2} C(\mathbf{q})\right)}
$$

If long-range order develops, it is evident that the Coulomb term becomes the Madelung 
energy associat $t$ with ordered charges on a Bravais lattice. In ordered alloys, the Madelung energy can, of course, be quite substantial. It should be evident from the above equations that the electrostatics can also be important for the SRO effects in the high-temperature alloy.

Indeed, as found by Johnson, St.lunton, and Pinski, the SRO in NiCr alloys results from a competition between a ciustering, band-filling $S_{\text {band }}^{2}(q)$ and an ordering, electrostatic contribution in the nickel-rich alloys [48]. The sum of those contributions yield an $\alpha(q)$ which has peaks at $\left(1, \frac{1}{2}, 0\right)$. This rather surprising result comes from the band-filling, $q=0$ peaks in $S^{2}$ being canceled from the tails of the electrostatic $q=\left(1, \frac{1}{2}, 0\right)$ peak, and the electrostatic peak being diminished, not canceled, (reducing the transition temperature) from the tails of the band-filling contribution to $S^{(2)}$. In $\mathrm{NiCr}$, then, there is a subtle cancellation between different electronic contributions giving rise to a robust result for the SRO.

\subsubsection{Magnetic Effezts: Ordering in $\mathrm{Fe}_{c} \mathrm{~V}_{1-c}$}

Before closing this section we make a few remarks regarding alloy phase stability in alloys that are magnetic. This is a large subject that we will not attempt to cover here. The progress that had been made within extensions of the KKR-CPA and CF approaches was the subject of the lectures of Prof. Staunton at the Alloy Phase Stability NATO ASI held in 1989 [42]. Thus, we will only review some results on the $\mathrm{Fe}_{c} \mathrm{~V}_{1-c}$ alloy system that have been obtained since that time and serve to highlight the quantitative nature of the theory and the importance of the magnetic effects in influencing the phase stability.

The generalization of the LDA-KKR-CPA method to alloy systems that are ferromagnetic using spin-polarized local density theory is quite straight forward and has produced results for the ground state total and partial magnetic moments that are in good quantitative agreement with magnetization and neutron scattering measurements [71], [72], [42], [73], [74]. Similarly, the concentration functional theory of ordering and phase stability can be generalized to the case of ferromagnetic systems. Here we assume that we are addressing the problem of chemical ordering in a system that is magnetically ordered i.e. the ordering/phase separation temperature is below the Curie temperature. In which case we have, at least formally, to consider the electronic grand potential to depend not only on the set of local concentrations $\left\{c_{i}\right\}$ but also the a set of local magnetic moments, $\left\{\mu_{i}\right\},[42]$. As a consequence, $S_{i j}^{(2)}$ consists of two terms [42], [75],[74]

$$
S_{i j}^{(2)}=S_{i j}^{c, c}+\sum_{l, \alpha} S_{i l}^{c, \mu i} \gamma_{o, l j}^{\alpha}
$$

where

$$
\begin{gathered}
S_{i j}^{c, c}=\left(\frac{\partial^{2} \bar{\Omega}^{C P A}\left(\left\{c_{i}\right\},\left\{\mu_{i}\right\}\right)}{\partial c_{i} \partial c_{j}}\right)_{c_{i}, c_{j}=\bar{c} V_{i}} \\
S_{i j}^{c_{i j} \mu_{j}=}\left(\frac{\partial^{2} \bar{\Omega}^{C P A}\left(\left\{c_{i}\right\},\left\{\mu_{i}\right\}\right)}{\partial c_{i} \partial \mu_{j}^{\alpha}}\right)_{c_{i}, c_{j}=\bar{\varepsilon} V_{i}} \\
\gamma_{i j}^{\alpha}=\left(\frac{\partial \mu_{i}^{\alpha}}{\partial c_{j}}\right)_{c_{i}, c_{j}=\bar{c} V_{i}}
\end{gathered}
$$

The new response fulction, $\gamma_{i j}^{a}$, measures the change in the magnitude of the local moment at site $i$ due to a change in the concentration at site $j$. Experimentally, both $\alpha(\vec{q})$ and $\gamma(\vec{q})$ can be measured using spin-polarized neutron scattering [76]. 


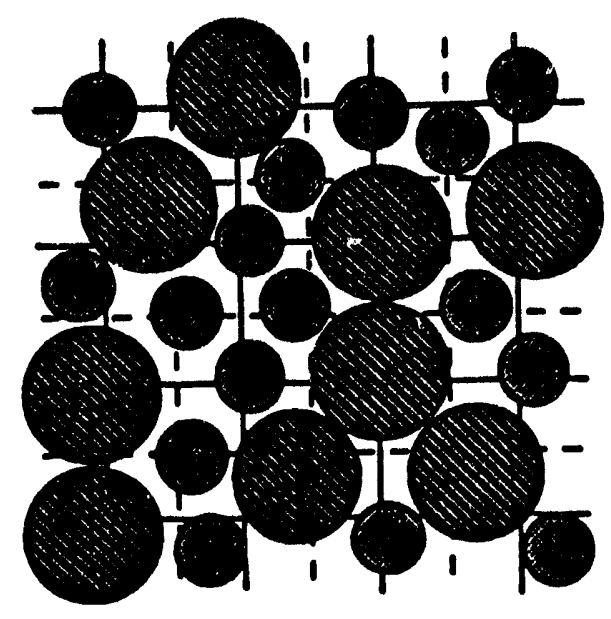

Figure 18: Warren-Cowley SRO parameter $\alpha(\overrightarrow{\mathbf{q}})$ along the [100](left) and [111] (right) directions in the bcc Brillouin zone for bcc ferromagnetic $\mathrm{Fe}_{0.863} \mathrm{~V}_{0.135}$. The triangles are the calculated $\alpha(\overrightarrow{\mathbf{q}})$ the crosses are the neutron scattering results of Cable et. al. [77]

Staunton et. al. have used the theory outlined above to calculate $\alpha(\overrightarrow{\mathbf{q}})$ in ferromagnetic $\mathrm{Fe}_{c} \mathrm{~V}_{1-c}$ alloys. One of their most striking results is shown in fig. 18 which for $\mathrm{Fe}_{0.865} \mathrm{~V}_{0.135}$ compares the calculated and experimental [77] $\alpha(\overrightarrow{\mathrm{q}})$ along the [100] and [111] directions in the bcc Brillouin zone. In addition to being in striking agreement with experiment the calculation can be pulled apart to provide an understanding of the origins of the various features in the curve. The zone boundary peak along the [100] direction in indicative of the $\mathrm{B} 2(\mathrm{CsCl})$ structure ground state. Along the [111]-direction the first peak is due to sort-ranged interactions out to the third neighbor, whilst the second peak results from a (111) Fermi surface nesting feature in the majority spin Fermi surface [75].

\subsubsection{Strain Fluctuations}

In all of the forgoing it has been assumed, teven in the disordered state, that the atoms occupy the sites of well defined underlying periodic lattice. Clearly, this will only be a good approximation for alloys for which in the language of Hume-Rothery the size difference is small. In alloys where this is not the case the average lattice is still periodic, as is made manifest by the fact that disordered alloys still have well defined X-ray diffraction Bragg scattering peaks, however, local relaxations occur. This situation is pictured schematically in fig 19 where we have envisioned the local lattice relations around large and small atoms. The lattice surrounding large atoms expands locally whilst the lattice surrounding small atoms responds by contracting.

As we have mentioned above [69], and, as has been discussed by others [78],[79], [80], [81] these local relaxation, or strain fluctuations, can give rise to important effects in alloy phase stability. Furthermore, they can now be measured very accurately using synchrotron radiation and anomalous scattering techniques [82],[83].

In a recent publication Gyorffy et. al. considered the problem of including strain fluctuation within the concentration functional theory outlined above [69]. Although no calculations have yet been performed that include strain fluctuations, for the sake of completeness we reproduce the major theoretical points here.

The theory is based on a straightforward generalization of the mean field theory 

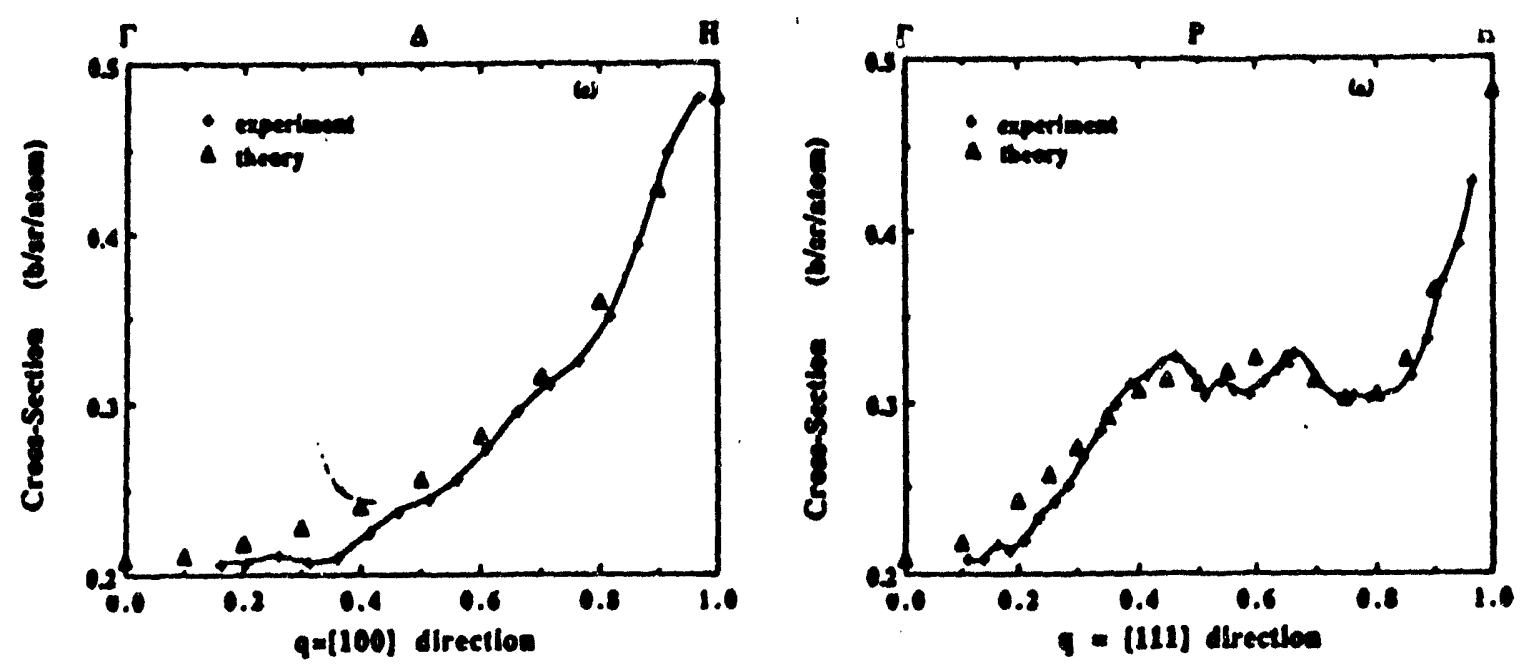

Figure 19: Schematic of local lattice relaxations around large and small atom on an underlying periodic lattice

described in section 3.2 in which they consider the electronic grand potential for the inhomogeneous concentration configuration $\left\{c_{i}\right\}$ on a relaxed lattice. That is to say each lattice point $\overrightarrow{\mathbf{R}}_{i}^{0}$ is assumed to have moved to the relaxed position $\overrightarrow{\mathbf{R}}_{i}=\overrightarrow{\mathbf{R}}_{i}^{0}+$ $\vec{u}_{i}$ under the influence of the compositional (Kanzaki) forces in order to minimize the free-energy $\Omega^{C P A}\left(\left\{c_{i}\right\},\left\{\vec{u}_{i}\right\}\right)$. Describing the total displacement field by the set $\left\{\vec{u}_{i}\right\}$, going through the arguments of Gyorffy and Stocks [20] and using the chain rule for differentiating with respect to the local concentrations, they find the following expression for the direct correlation function

$$
S_{i j}^{(2)}=S_{i j}^{c, c}+\sum_{l, a} S_{i l}^{c, u_{i}^{a}} \gamma_{j, j j}^{a}
$$

where $\alpha=x, y$ and $z$ and

$$
\begin{gathered}
S_{i j}^{c, c}=\left(\frac{\partial^{2} \bar{\Omega}^{C P A}\left(\left\{c_{i}\right\},\left\{\vec{u}_{i}\right\}\right)}{\partial c_{i} \partial c_{j}}\right)_{c_{i, c}=\bar{c} V_{i}} \\
S_{i j}^{c, u_{j}^{\alpha}}=\left(\frac{\partial^{2} \tilde{\Omega}^{C P A}\left(\left\{c_{i}\right\},\left\{\vec{u}_{i}\right\}\right)}{\partial c_{i} \partial u_{j}^{\alpha}}\right)_{c_{i,}, c_{j}=\varepsilon V i} \\
\gamma_{s, i j}^{\alpha}=\left(\frac{\partial u_{i}^{\alpha}}{\partial c_{j}}\right)_{c_{i}, c_{j}=\bar{c} V_{i}}
\end{gathered}
$$

The effects of the strain fluctuations on the short range order are described by the new response functions $S_{i j}^{c, u}$, and $\gamma_{,, i j}^{\alpha}$. Gyorfy et. al. developed a theory for $\gamma_{,, i}^{a}$ in the limit where the displacements, $\left\{\tilde{u}_{i}\right\}$ are regarded as small and their effect on $\Omega^{\text {teA }}$ is taken into account only in the Harmonic approximation. Under these assumptions
they found

$$
\gamma_{a, i j}^{\alpha}=\sum_{l, \beta} \Phi_{\alpha \beta}^{-1}(i, l) S_{l i}^{c, u}
$$

where the static force constants $\Phi_{\alpha \beta}(i, j)$ are given by

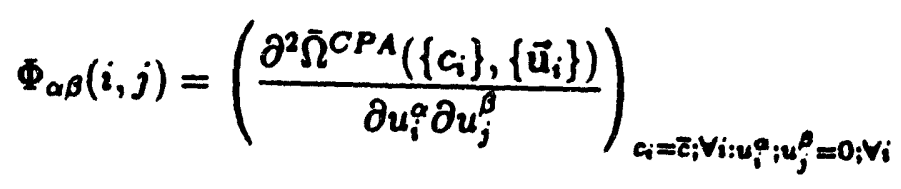




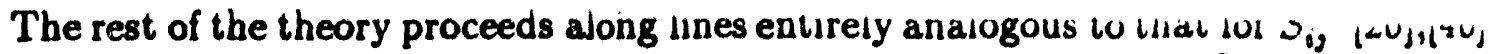
and the end results are complicated response function formulas (for $S_{i j}^{c, w_{j}^{0}}$ and $\Phi_{a \beta}(i, j)$ ) which have to be evaluated using the results of a LDA-KKR-CPA calculations for a homogeneous solid solution with concentration $\bar{c}$. Choosing the homogeneous solid solution as the reference state and lattice Fourier transforming eq. 56 leads to

$$
S^{(2)}(\overrightarrow{\mathbf{q}})=S^{c, c}(\overrightarrow{\mathbf{q}})+\sum_{\alpha} S^{c, v^{\alpha}}(\overrightarrow{\mathbf{q}}) \gamma_{\bullet}^{\alpha}(\overrightarrow{\mathbf{q}})
$$

Clearly, $S^{(2)}(\vec{q})$ picks up contribution from the stain fluctuations (in this respect the situation is entirely analogous to the magnet contribution discussed above). It was this stain fluctuation contribution to which we appealed to resolve the dilemma regarding $\mathrm{Ni}_{c} \mathrm{Pt}_{1-c}$ alloys discussed above. Thus, when implemented, this theory should cast light on a number of interesting alloying phenomena as well as providing a interpretation for the growing body of experimental measurements of local lattice relaxations in alloys obtained using anomalous X-Ray scattering [82],[83].

It is worth reiterating one of the points made by Gyorffy et. al. [69] with regard to the way that the strain fluctuations have been included. Given the way elastic forces propagate in solids the strain fluctuation contribution to $S^{(2)}(\vec{q})$ is bound to be lon/s ranged. Indeed, in the elastic dipole limit, we may expect contributions which fall off as $S_{i j}^{(2)}=\left|\vec{R}_{i j}\right|^{-3} \exp \left(-\zeta / \overrightarrow{\mathbf{R}}_{i j}\right)$. From this point of view, the first principles mean-field theory method advocated here is particularly promising since it requires calccistions in $\overrightarrow{\mathbf{q}}$-space and treats the small and large $\overrightarrow{\mathbf{q}}$ limits on an equal footing. In this respect, it is quite different from supercell or finite cluster based methods [84]. These considerations are also relevant to the applicability of the Connolly-Williams type of approaches. Clearly, to account for large elastic interactions, the effective Hamiltonian must include long-ranged forces for which both the CVM and the Monte Carlo method become difficult to implement. Fortunately, as was recently demonstrated by Marias et al. [85], under these circumstances the mean-field theory becomes a better and better approximation.

\subsection{Generalized Perturbation Method}

The GPM is based on a perturbation expansion of the electronic energy associated with specific alloy configurations about the reference medium provided by the homogeneously disordered alloy $[18,86]$. Thus, for some specific configuration of the alloy $\left\{\xi_{i}\right\}$, the electronic grand potential $\Omega\left\{\xi_{i}\right\}$ is decomposed into a concentration dependent but configuration independent contribution $\bar{\Omega}(c)$ and a composition and configuration contribution $\Omega^{\prime}\left(\left\{\xi_{i}\right\}\right)$ as

$$
\Omega\left\{\xi_{i}\right\}=\bar{\Omega}(c)+\Omega^{\prime}\left(\left\{\xi_{i}\right\}\right) .
$$

As is illustrated in fig 20 the homogeneously disordered alloy is a sensible reference medium in that the energy difference between the disordered state and the energy of any short ranged ordered state and globally ordered states will, in general, be small. In systems that undergo order-disorder transformations this is clearly the case since this energy is the order of the ordering temperature which typically are in the range 0-2000K (0-13 mRy/atom), which can be compared with $t$ pical cohesive energies of metals which are in the range $100-700 \mathrm{mRy} /$ atom [62].

Since the LDA-KKR-CPA method provides us with a first principles theory of the energetics of the homogeneously disordered phase it makes sense to make the association $\bar{\Omega}(c) \Rightarrow \Omega^{C P A}(c)$ where $\Omega^{C P A}(c)$ is the LDA-KKR-CPA approximation to the electronic grand potential of the homogeneously disordered alloy. 


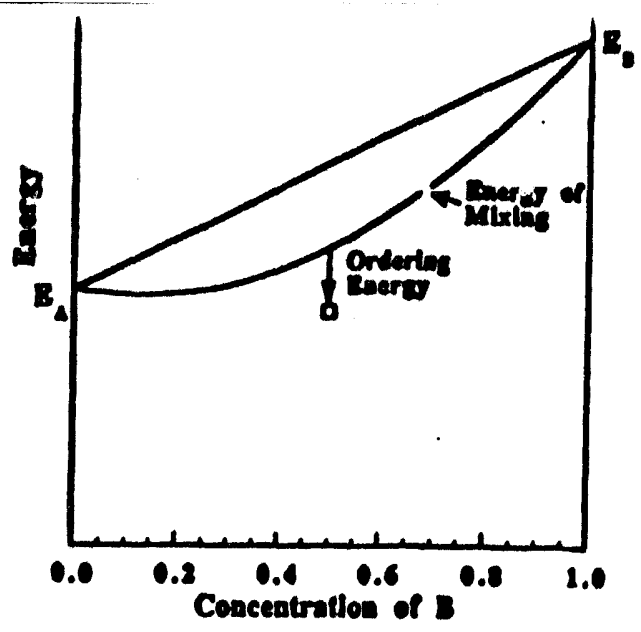

Figure 20: Schematic representation of the energy of mixing and of some particular ordered phase as a function of the atomic fraction of the B-species

A detailed derivation of the GPM method and the related embedded cluster method (ECM) is given in references [87, 19]. Reference [88] compares the GPM method with the Connolly Williams method devoloped in the lectures in this volume by Dr. Zunger. We will not repeat the derivations here but will simply outline the method, quote the important formulae, and show some illustrative results.

As in previous section we will assume that for the electron system it is sufficient to work at $\mathrm{T}=0 \mathrm{~K}$. Whence, $\Omega^{C P A}=E^{C P A}-\mu N$ where $E^{C P A}$ is the LDA-KKR-CPA energy of the random alloy eq. $32, \mu$ is the electron chemical potential and $N=N\left(\epsilon_{F}\right)$ is the integrated density of states at the Fermi energy $\mu=\epsilon_{F}$. The configuration dependent contribution of the energy can be written as an expansion in concentration dependent $n^{\text {th }}$-order effective cluster interactions, $V_{i j . . . n}^{n}$ as

$$
E\left(\left\{c_{i}\right\}\right)=E^{C P A}(c)+\frac{1}{2 !} \sum_{i j} V_{i j}^{2} \delta c_{i} \delta c_{j}+\frac{1}{3 !} \sum_{i j k} V_{i j k}^{3} \delta c_{i} \delta c_{j} \delta c_{k}+\ldots
$$

where $\delta c_{i}=\xi_{i}-c$. The many-site interchange potentials $V_{i j}^{2}, V_{i j k}^{3}$ etc. are then given in terms of quantities that are available at the end of a KKR-CPA calculation of the random solid solution [87]. [19]. If we consider only the band structure energy contribution to $E^{C P A}$

$$
E^{C P A}-\mu N(\mu)=-\int_{-\infty}^{\infty} d \epsilon N(\epsilon, \mu) \Theta(\epsilon-\mu)
$$

the $n^{\text {th }}$-order effective cluster interaction is given by

$$
V_{i j \ldots n}^{n}=-\frac{2}{\pi} \operatorname{Im} T r\left[\int_{-\infty}^{\infty} d \epsilon \Theta(\epsilon-\mu)\left(\Delta X_{1} \tau^{c, 12} \Delta X_{2} \tau^{c, 23} \ldots \Delta X_{n} \tau^{c, n 1}\right)\right]
$$

where the quantity $\Delta X_{n}$ gives a measure of the on site interaction which involves the difference in scattering strengths of $A$ and $B$ impurities embedded in a CPA lattice. It is given by

$$
\Delta X_{n}=X_{n}^{A}-X_{n}^{B}
$$

with

$$
X_{n}^{\alpha}=\left[1-\left(t_{c, n}^{-1}-t_{\alpha, n}^{-1}\right) \tau^{c, n n}\right]^{-1}\left(t_{c, n}^{-1}-t_{\alpha, n}^{-1}\right)
$$

In fig. 21 we illustrate schematically the conten، of eq. 66 . For example the $2^{\text {nd }}$ order pair wise interaction $V_{0,1}^{(2)}$ consists of an interaction at site 0 propagating in the CPA medium to site 1 , interaction at site 1 , and returning to site 0 . 


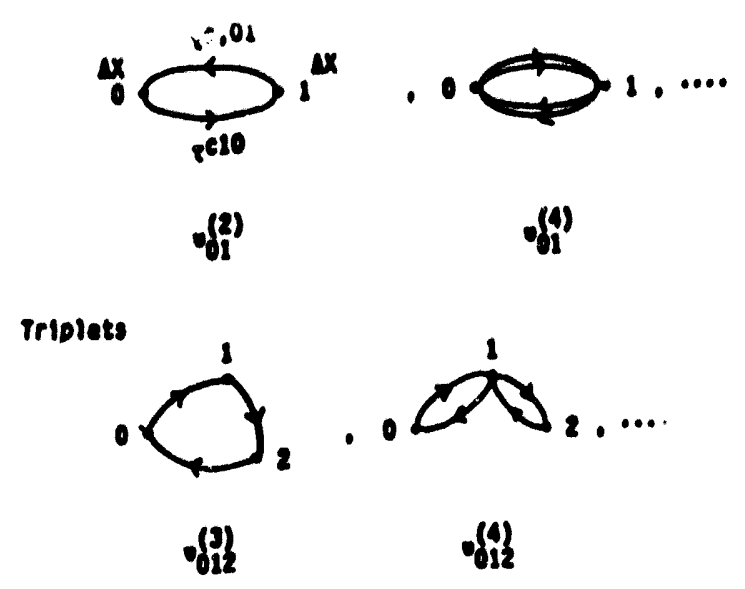

Figure 21: Schematic representation of the way the GPM-multisite interactions are built up for the second and fourth order pairwise interaction and the third and fourth order triplet interactions.

Equations 66, 67 and 68 neglect contributions to the interchange potentials that result from consideration of the double counting terms in $E^{C P A}$. Again this approximation should be valid in systems where charge transfer is small. In most of the example systems considered below this is the case. The one exception is NiAl and we will comment on the consequences of this laier.

It should be noted that nowhere do we build in any explicit approximation to either the concentration dependence, the complexity (pair, triplet,...), or the range of the interactions other than that implicit in the CPA. Thus these factors have to be investigated for each alloy system considered. In fig. 22 we show illustrative results
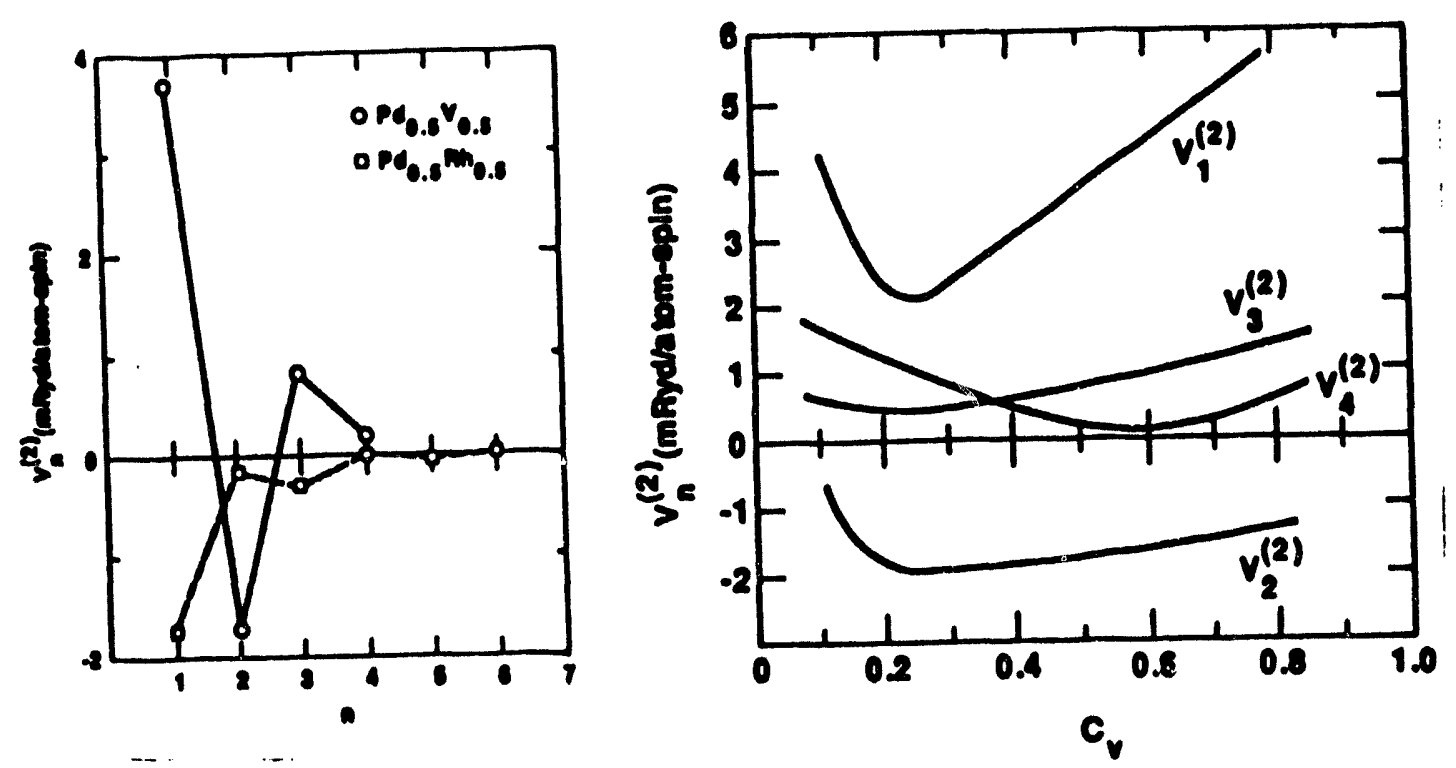

Figure 22: Range of pairwise interactions in $\mathrm{Pd}_{c} \mathrm{~V}_{1-c}$ and $P \mathrm{Pd}_{c} \mathrm{Rh}_{1-c}$ alloys.

for the concentration dependence of the pair wise interactions out to fourth nearest neighbors $P \dot{\mathrm{d}}_{(1-c)} \mathbf{R h}_{c}$ and the range dependence at fixed composition for $\mathrm{Pd}_{0.8} \mathrm{~V}_{0.5}$ and $\mathbf{P d}_{0.3} \mathrm{~V}_{0.3}$ all on a underlying fcc lattice [87]. Clearly, in $\mathbf{P d}_{(1-c)} \mathrm{V}_{c}$ all of the interactions are strongly concentration dependent. At $c=0.5$ the pairwise interaction converge rapidly for both alloy systems. For PdV (PdRh) the first neighbor interactions are ordering (phase separating) consistent with the phase diagram and for PdRh with the 


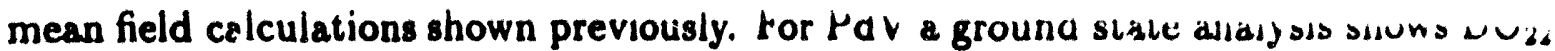
to be the stable structure for $c=0.25$ as is observed experimentally. At $c=0.5$ the calculations fayor an $\mathrm{Ll}_{0}$ structure whilst the published phase diagrams show a solid solution. However, a B19 structure has been reported [87]. Interestingly, this structure can be thought of as a monoclinic distortion of the $\mathrm{Ll}_{0}$ structure. For most of the systems that have been investigated the triplet and higher order interactions are generally small. However there are some notable exceptions. For example in fcc $\mathrm{Ni}_{0.78} \mathrm{Al}_{0.23}$ the nearest neighbor triplet interaction is $2.6 \mathrm{mRy} / \mathrm{atom}$. Whilst, this is small compared with the nearest neighbor pair interaction (14.2 mRy/atom) it is large compared with the second (-1.0 mRy/atom) and third (-1.7 mRy/atom) neighbor pairwise interactions.

In the remainder of this subsection we will show the results for calculated phase diagrams for two model systems $\mathrm{Cu}_{(1-c)} \mathrm{Zn}_{c}$ [32] and $\mathrm{Al}_{(1-c)} \mathrm{Ni}_{c}$ [89] These systems serve to illustrate some of the successes and some of the remaining difficulties with the current implementation of the GPM method that neglects the double counting contributions.

\subsubsection{Phase Stability of Cu-rich CuZn Alloys}

The $\mathrm{Cu}_{(1-c)} \mathrm{Zn}_{c}$ alloy system has been one of the most studied and is an example of one of the Hume-Rothery electron compounds. Hume-Rothery related the position of the $\alpha-\beta$ (fcc-bcc solid solution) two phase boundary to the electron to atom ratio $\zeta$.

Figure 23 shows the effective pairwise interactions for fcc and bcc alloys. These interactions correspond to the energies of mixing shown in fig. 7. For both bcc and fcc, the interactions show a strong ordering tendency at all concentrations. Interactions more distant than second neighbor are an order of magnitude smaller than those shown.

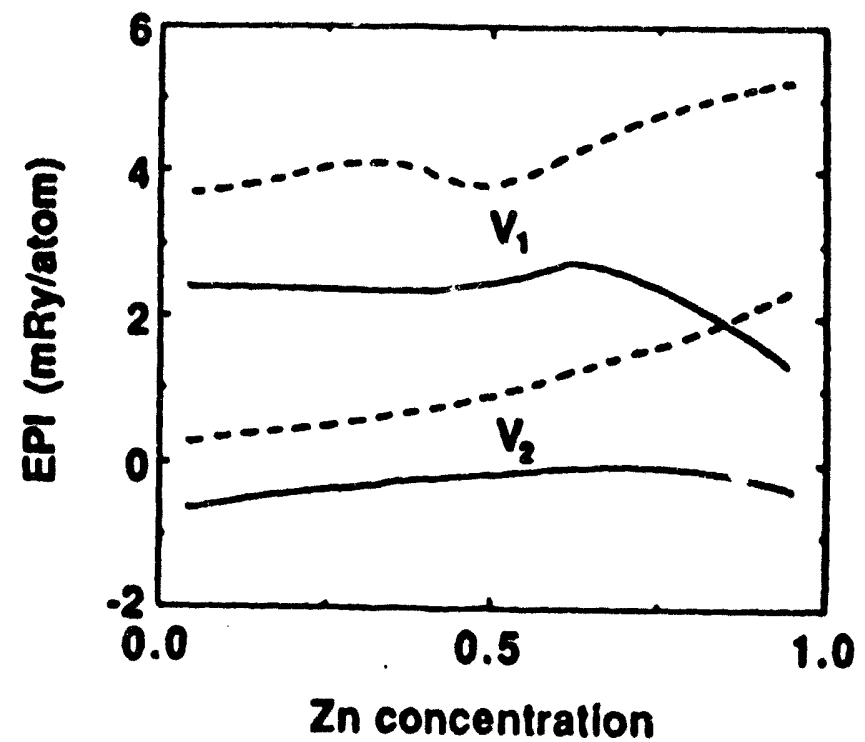

Figure 23: Concentration dependence of the first $V_{1}$ and second $V_{2}$ neighbor pairwise interactions for fcc- (solid) and bcc- (dashed) based structure $\mathrm{Cu}_{(1-c)} \mathrm{Zn}_{c}$ alloys.

A ground state analysis including pairwise interactions out to fourth (fifth) neighbor fcc (bcc) indicate that the $\mathrm{Ll}_{2}\left(\mathrm{DO}_{3}\right)$ structure are stable at $c=0.25$ and 0.75 and the $\mathrm{Ll}_{0}$ (B2) structure is stable at $c=0.5$. Figure 24 shows the calcrilated [32] and assessed [33] $\mathrm{Cu}_{(1-c)} \mathrm{Zn}_{c}$ phase diagrams. The calculated phase diagrarn was obtained using the tetrahedron-octahedron (irregular tetrahedron) epproximation CVM for fcc 
(bcc) based alloys using the interactions sbown in hg. 23 and a semi-empirical cuisce. tion for the vibrational entropy based on Debye theory [32]. The latter only affects the phase diagram for temperatures $>400^{\circ} \mathrm{C}$. At least for $\mathrm{Cu}$ rich alloys where the observed
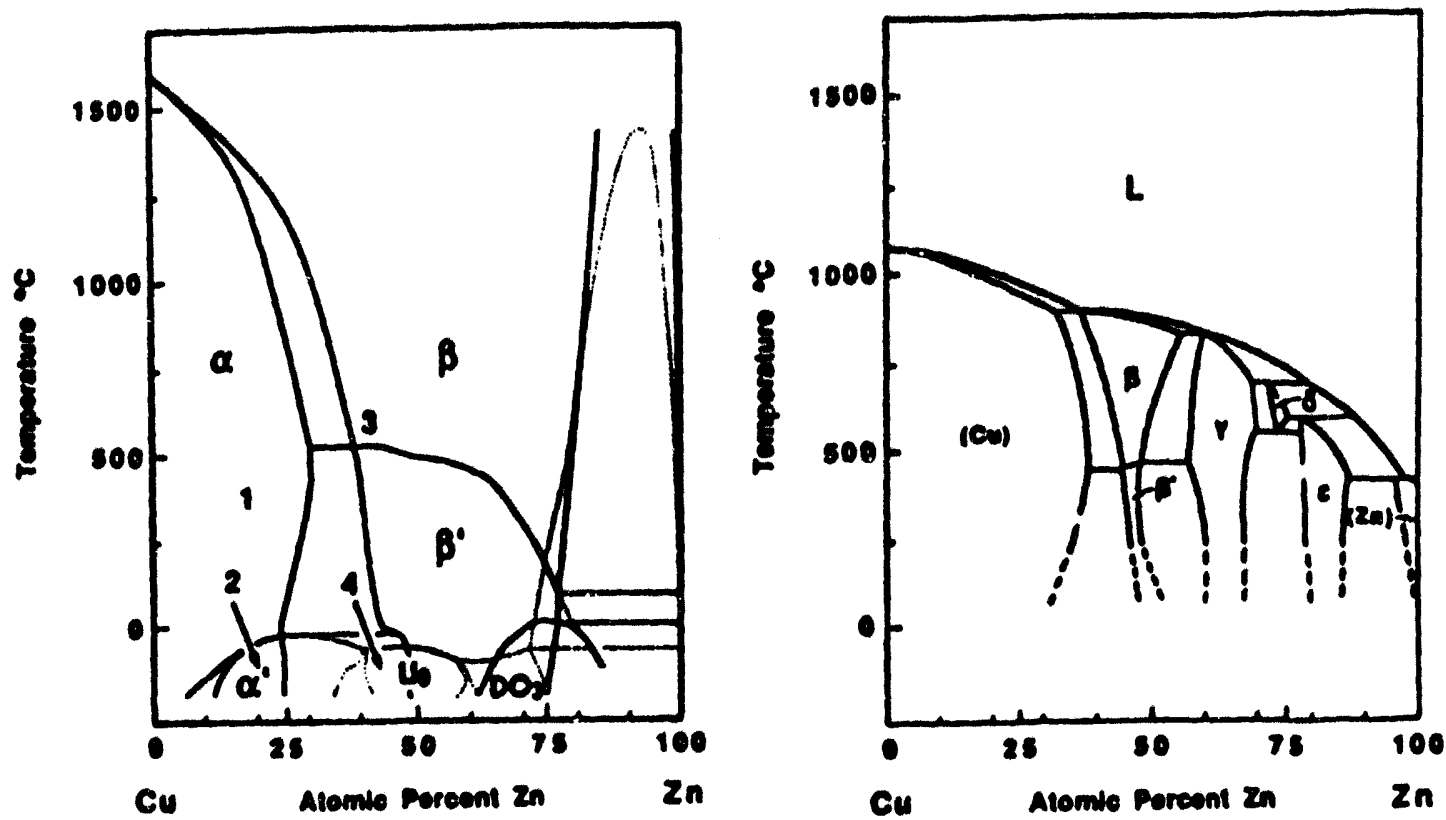

Figure 24: Theoretical $\mathrm{Cu}-\mathrm{Zn}$ phase diagram (left) compared with the asseased one (right). The dotted lines indicate meiastable boundaries.

phases are either fcc or bcc based, regions accented by the bold numbers, the calculated phase diagram bears a striking resemblance to the assessed one. There are a couple points worthy of mentioning here. Firstly, at $c=0.5$ the calculated order-disorder phase transformation temperature $\left(510^{\circ} \mathrm{C}\right)$ between ordered $\mathrm{B2}\left(\beta^{\prime}\right)$ and disordered bcc $(\beta) \mathrm{CuZn}$ round $c=0.5$ is in good agreement with experiment $\left(\sim 470^{\circ} \mathrm{C}\right)$. Secondly, the convex curvature of the $\alpha-\beta$ two phase field results from the inclusion of the vibrational entropy, without this semi-empirical correction the two phase field boundaries are essentially vertical [32]. There are a number of other interesting features of the calculated phase diagram, for a detailed discussion of these reader is referred to $[32]$ and [90].

\subsubsection{Phase Stability of Ni-rich NiAl Alloys}

During the last few years $\mathrm{NiAl}$ alloys have been at the center of a great deal of alloy development work based on modifications to two of the ordered compounds $\mathrm{Ll}_{0}$ structure $\mathrm{Ni}_{3} \mathrm{Al}$ and $\mathrm{B2}$ structure $\mathrm{NiAl}$. Although single crystals of $\mathrm{Ni}_{3} \mathrm{Al}$ are quite ductile as cast polycrystalline material is extremely brittle but can be ductilized by the addjtion of a few hundred parts/milition of boron. B2 structure NiAl, however, has 80 far resisted all attempts to make it ductile in any useful way. In addition to being a candjdate structural material, $\mathrm{B} 2 \mathrm{NiAl}$ is also interesting as a possible shape memory alloy. For $\mathrm{Ni}$ concentrations around 62.5 percent $\beta$-phase $\mathrm{NiAl}$ transforms Martensitically to the $7 \mathrm{R}$ structure. It is observed that the transition temperature is very composition dependent ranging from $0 \mathrm{~K}$ for $c \sim 0.60$ to well above room temperature for $c \sim 0.66$. Consequently, these alloys are interesting candidates as controllable transition temperature shape memory alloys. In addition to being interesting engineering materials these alloys exhibit a number of interesting pre-Martensitic phenomena. As a result, 
they have become one of the model systems for studying Martensitic transformation. We shall have more to say on this in 4.2.

In figure 25 we show the calculated results for the LDA-KKR-CPA mixing energies and the corresponding GPM first and second neighbor pairwise interactions for both fcc and bcc NiAl alloys [89]. In addition the formation energies of ordered structures
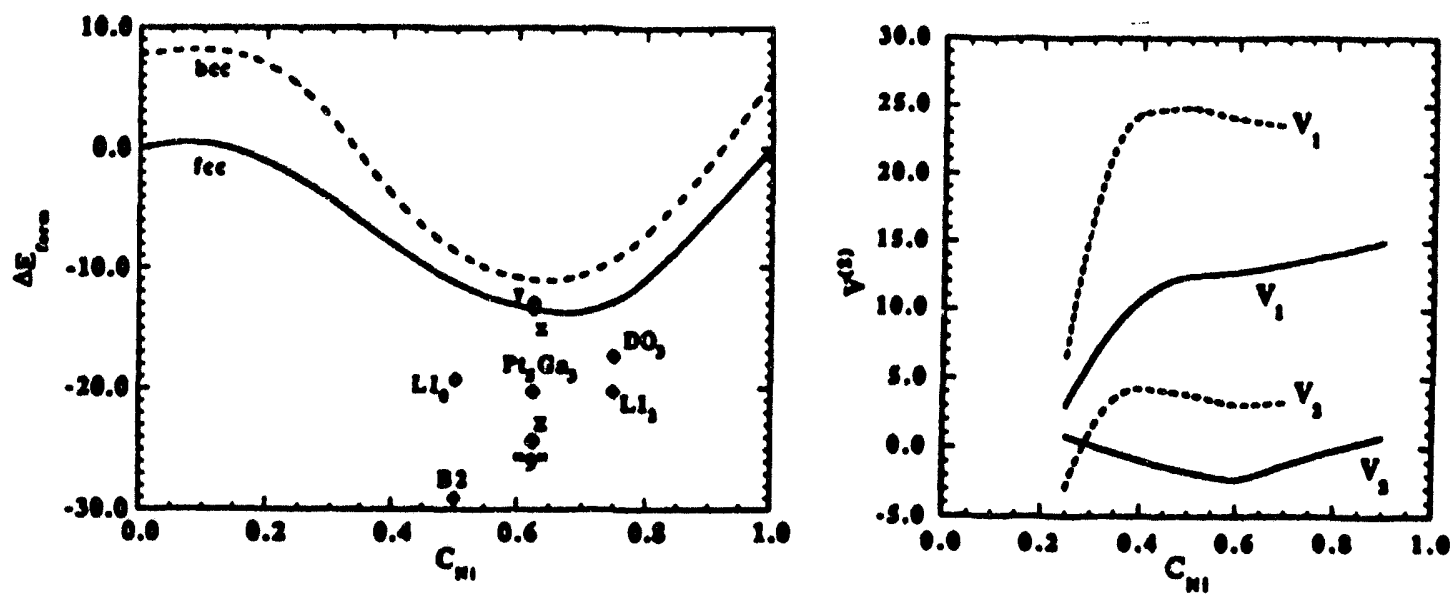

Figure 25: Concentration dependence of the LDA-KKR-CPA energies of mixing (left frame) and GPM first $\left(V_{1}\right)$ and second $\left(V_{2}\right)$ neighbor pairwise interactions for fcc- (solid) and bcc- (dashed) based structure $\mathrm{Al}_{(1-c)} \mathrm{Ni}_{c}$ alloys. The various points identified by structural notation are the formation energies corresponding to that structure.

that result from a ground state analysis using the GPM pair wise interactions together with the energies of mixing are also displayed. In referencing the mixing energies of the fcc and bcc structures to one another we used the empirical structural energy differences between fec and bec of Saunders et al. [91] (7.68 mRy/atom for Ni, 5.70 $\mathrm{mRy}$ for $\mathrm{Al}$ ) for the end points with a linear interpolation between. The structural energy differences for $\mathrm{Ni}$ and $\mathrm{Al}$ calculated with the muffin-tin approximation LDAKKR-CPA code are smaller than the semi-empirical ones (3.46 mRy/atom for $\mathrm{Ni}, 3.50$ $\mathrm{mRy}$ for $\mathrm{Al}$ ). Since the muffin-tin approximation is notoriously poor when used to calculate structural energy differences we opted for the semi-empirical values. We note that use of the calculated values results in a phase diagram that is much worse than that shown below.

For Ni-rich alloys, the experimentally observed structures [33] are orderings on fcc or bcc lattices or, as in $\mathrm{Ni}_{3} \mathrm{Al}_{3}$, a closely related structure. A careful analysis of the ground state energies obtained using the GPM interactions (see fig.25)reveals that at $c=0.5 \mathrm{bcc}$ based $\mathrm{B} 2$ is stable with respect to fcc based L1。 whilst at $c=0.75 \mathrm{fcc}$ based $\mathrm{Ll}_{2}$ is stable with respect to bcc based $\mathrm{DO}_{3}$ [92], [89]. At $\mathrm{c}=0.625$ the most stable phase is, in the notation of Finel and Ducastelle [93], phase 9. However, closer examination reveals that this structure is closely related to the experimentally observed structure of $\mathrm{Ni}_{5} \mathrm{Al}_{3}$ namely $\mathrm{Pt}_{5} \mathrm{Ga}_{3}$. The $\mathrm{Pt}_{3} \mathrm{Ga}_{3}$ structure is a bet structure with a c/a-ratio intermediate $(c / a=1.24)$ between that of bcc $(c / a=1.0)$ and $f c c(c / a=\sqrt{2})$ when the latter is viewed as bct.

In fig. 26 we reproduce the phase diagram calculated by Sluiter et al. [89] using the cluster variation method (CVM) [94] with calculated GPM fcc and bcc interchange energies and KKR-CPA heats of mixing together with experimental structural energy differences [95]. The calculated phase diagram closely resembles the experimental one [33]. The B2-L1。 two phase region is in good agreement with experiment, the calculated order-disorder temperature of the $\mathrm{Ll}$ 。 phase is $1770^{\circ} \mathrm{C}$ which is just abore the melting point as was surmised by Cahn et al. [96]. Of course the liquid phase and the ordered 


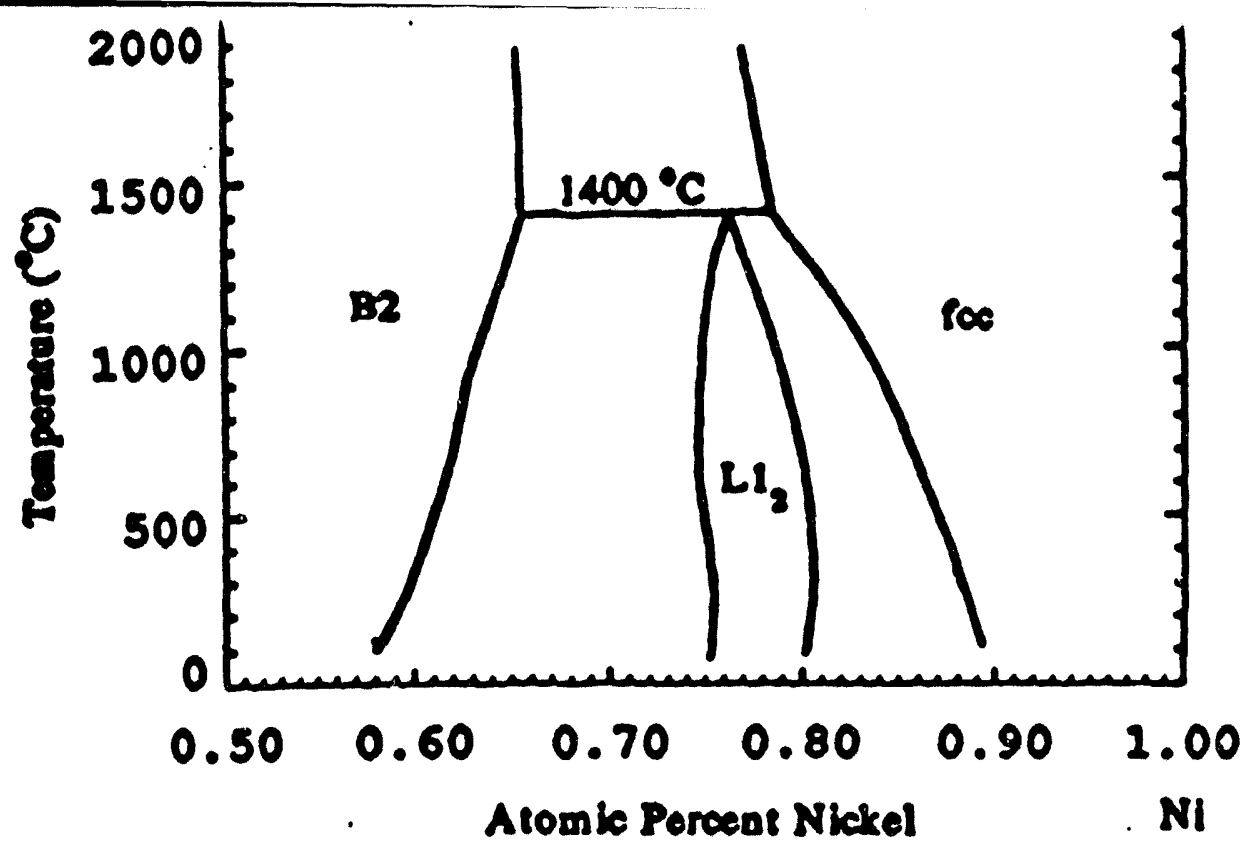

Figure 26: Ni-rich portion of the Ni-Al phase diagram computed by Sluiter et al..

$\mathrm{Ni}_{5} \mathrm{Al}_{3}$ phase are both missing from the calculated phase diagram since neither can be represented by the current GPM-CVM methods. It should be noted that the calculated order/disorder temperature for the B2/bcc phases of $5345^{\circ} \mathrm{C}$ is much greater than the melting temperature of the B2 phase.

\section{Complex Lattice Alloys}

Up to this point we have only considered alloy systems that can be treated as solid solutions on an underlying simple lattice (fcc, bcc). In this final section we turn our attention to alloys where it is necessary to consider systems as being composed of several underlying sub-lattices. Some of the many situations where this is appropriate are illustrated schematically in fig. 27. These include states of partial long range order, off-stoichiometric compounds, and binary compounds with ternary additions. In the following we will refer to these as complex lattice or multi-sublattice alloys.

The extension of the LDA-KKR-CPA method described in section 2.1 to complex lattices is straight forward [97], [98], [99]. the central point is that the single site effective $t$-matrix $t_{C}^{*}$ now depends on the sublattice index $s$. However, as befits the single site nature of the CPA $t_{C}^{*}$ is diagonal in the site index. Thus, the KKR-CPA equations for each sublattice, $s$, take the form

$$
\sum_{\alpha} c_{\alpha}^{\circ} \tau_{\alpha, L L^{\prime}}^{n n, \rho}(\epsilon)=\tau_{c, L L^{\prime}}^{n n, \rho}(\epsilon)
$$

where $\tau_{c, L L^{\prime}}^{n n,}(\epsilon)$ is the $(n, n)$-th element of the effective CPA medium's scattering path matrix for the s-th sub-lattice, $\tau_{\alpha, L L^{\prime}}^{n n, 0}(c)$ is the scattering path matrix for the effective CPA medium with the effective $t$-matrix $t_{c}^{*}$ at the $n$-th site replaced by the $t$-matrix, $t_{\alpha}^{s}$, corresponding to a real $\alpha$-type scatterer. The index $\alpha$ runs over the different type of atoms on the s-th sublattice and $c_{\alpha, a}$ is the concentration of the $\alpha$-th species on the $s$-th sub-lattice. The concentrations $c_{\alpha, 0}$ are defined such that

$$
\sum_{a} c_{\alpha}^{0}=1
$$



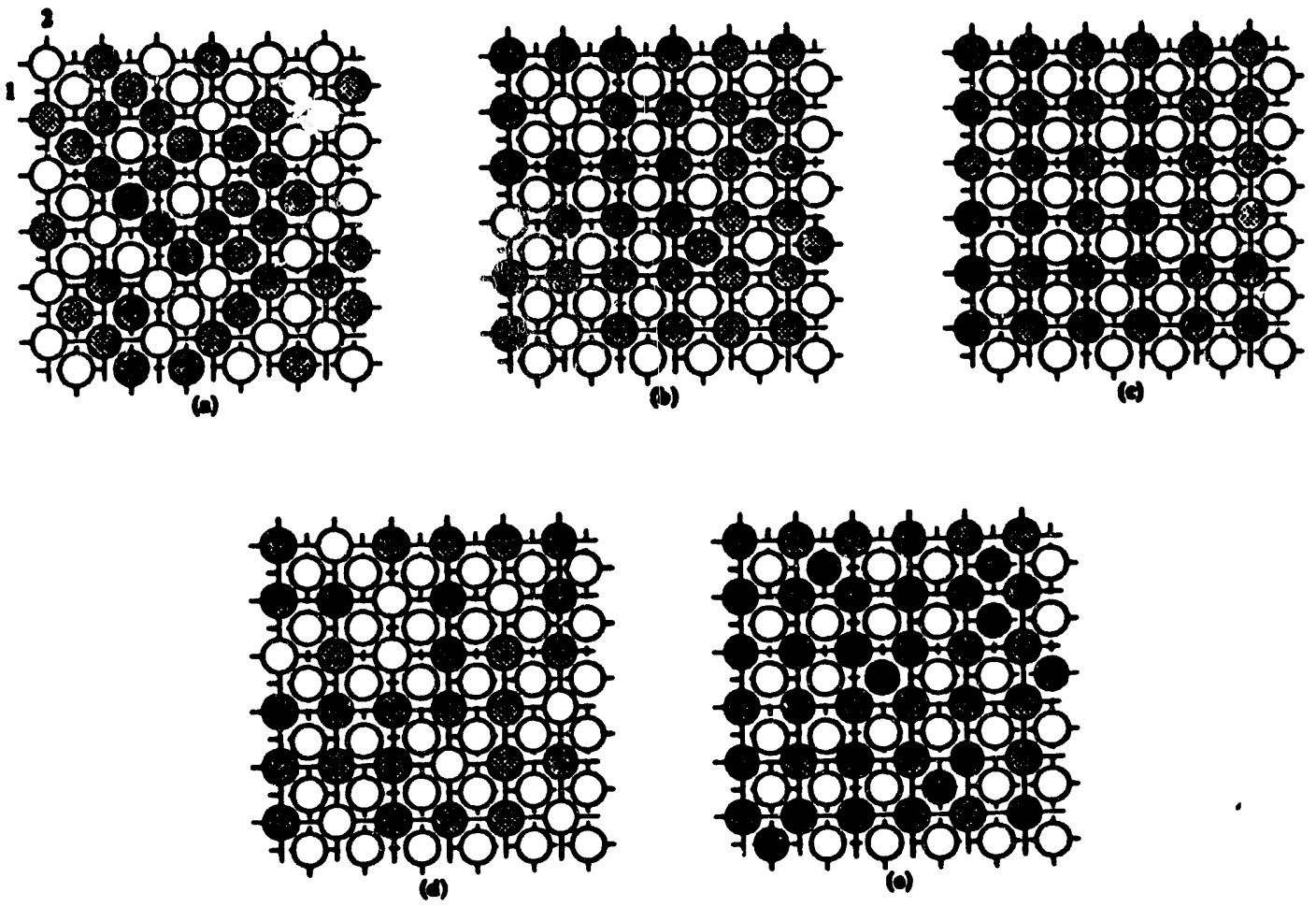

Figure 27: Two dimensional depiction of a binary $A_{c} B_{(1-c)}$ alloy. We have identified two sublattices $(1,2)$ and two atomic species ( $A \equiv$ unshaded; $B \equiv$ shaded), (a) disordered $A_{0.5} B_{0.5}$ solid solution for which the long range order parameter $\eta=0,(b)$ a partially ordered alloy $0 \leq \eta \leq 1$ (c) ordered AB intermetallic compound $\eta=1$, (d) off stoichiometric compound in which the excess $A$ atoms form antisite defects on the second (B) sublattice, (e) addition of a third alloying element to a ordered binary compound 
The form of the remaining KKR-CPA equations 14 and 16 is unchanged trom ine singse sub-lattice case excepting that all of the matrices are now matrices in site and angular momentum indices e.g. $G(\vec{k} ; \epsilon)$ has elements $G_{L_{L^{\prime}}^{\prime \prime}}^{\prime \prime}$

In the remainder of this section, we will summarize the results of applying the complex lattice LDA-KKR-CPA to $\beta$-phase $\mathrm{Ni}_{e} \mathrm{Al}_{1-c}$ alloys. In the next subsection we consider the ordering energy and the evolution of the ordering energy as a function of the long range order (LRO) parameter. In the final subsection we study the electronic structure and Fermi surface of off-stoichiometric NiAl alloys in the concentration range where the $\beta$-phase undergoes a Martensitic phase transformation and comment on the possibility that the pre-Martensitic phenomena that are osbserved in this system are driven by Fermi surface nesting as recently suggested by Zhao and Harmon [100].

\subsection{Ordering Energies}

Although the energy difference between the ideal disordered phase of an alloy and a related ordered phase is not generally experimentally measurable it does provide an important measure of the strength of the ordering interactions present in the system. The order-disorder energy $\Delta E^{0-d}$ for a binary $A_{c} B_{(1-c)}$ may be defined as

$$
\Delta E^{\circ-d}(X, x)=E^{\circ}(X)-E^{d}(x, c)
$$

where $x$ refers to the structure of the disordered lattice and $X$ refers to some ordered structure based on this lattice and $E^{d}(x, c)$ and $E^{\circ}(X)$ are the corresponding ground state $\left(T=0^{\circ} \mathrm{K}\right)$ energies. Clearly, when $\Delta E^{\circ-d}$ is large the alloy is strongly ordering and the order-disorder temperature is high.

As is pictured schematically in fig 27 we treat the ideal stoichiometric NiAl B2 $(\mathrm{CsCl})$ structure ordered phase as two interpenetrating simple cubic lattices having sub-lattice compositions $\mathrm{Ni}_{1.0} \mathrm{Al}_{0.0}$ and $\mathrm{Ni}_{0.0} \mathrm{Al}_{1.0}$. In order to minimize the relative error when subtracting the large energies of the individual phases we also treat the disordered phase $\mathrm{Ni}_{0.5} \mathrm{Al}_{0.5}$ as comprising two interpenetrating simple cubic lattices each with composition $\mathrm{Ni}_{0.5} \mathrm{Al}_{0.3}$.

In fig. 28 we show the energies of bcc disordered $\mathrm{Ni}_{0.5} \mathrm{Al}_{0.5}$ and ordered $\mathrm{B2}$ structure $\mathrm{NiAl}$. In fig. 28 the minima of the energy versus lattice spacing curves give the ground state energies, $E^{\circ}(B 2)$ and $E^{d}(b c c, c)$ respectively, the difference, $E^{\circ}(B 2)-E^{d}(b c c, c)$, then gives the order-disorder energy $\Delta E^{\circ-d}(B 2, b c c)=47.7 \mathrm{mRy} / \mathrm{atom}$. The minima in the two curves occur at different lattice spacings. The predicted equilibrium lattice spacings are 5.45 a.u. and 5.40 a.u. for the disordered and ordered phases respectively, corresponding to a volume expansion of approximately $3 \%$ on disordering. The experimentally determined lattice spacing for stoichiometric $\mathrm{NiAl}$ is 5.455 a.u. [101].

The LDA-KKR-CPA not only allows one to treat the fully disordered and fully ordered states but also opens up the possibility to study the evolution of the energy as a function of the long range order (LRO) parameter. If we consider the disordering of $\mathrm{NiAl}$ by random exchanges of $\mathrm{Ni}$ and $\mathrm{Al}$ atoms between the $\mathrm{Ni}$ and $\mathrm{Al}$ sub-lattices, as pictured schematically in fig. 27 , we may model the LRO state by two interpenetrating simple cubic sub-lattices having compositions $\mathrm{Ni}_{(1-c)} \mathrm{Al}_{c}$ and $\mathrm{Ni}_{c} \mathrm{Al}_{(1-c)}$ for concentrations $c$ in the range $0 \leq c \leq 0.5$. If we define the LRO parameter as $\eta=(2 c-1)$ then $\eta=1, c=1$ corresponds to the ordered $\mathrm{B} 2$ structure and $\eta=0, c=0.5$ corresponds to the homogeneously disordered state.

In fig. 29 we show the energy of $\mathrm{Ni}_{0.5} \mathrm{Al}_{0.5}$ plotted as a function of the LRO parameter. The zero of energy is taken to be the energy of the disordered state. It is interesting that the $\eta$ dependence of the ordering energy departs strongly from the quadratic dependence that would be expected if the interactions were entirely pairwise. 


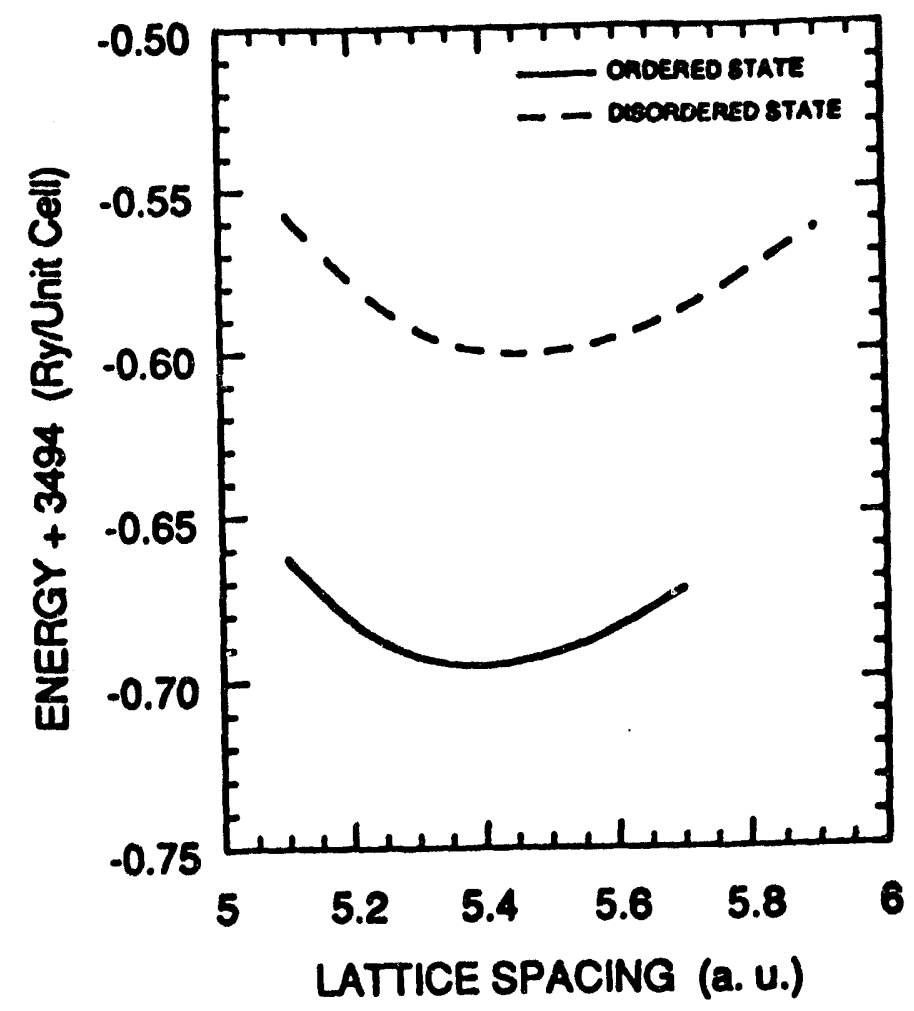

Figure 28: Ground state energies of ordered and disordered NiAl

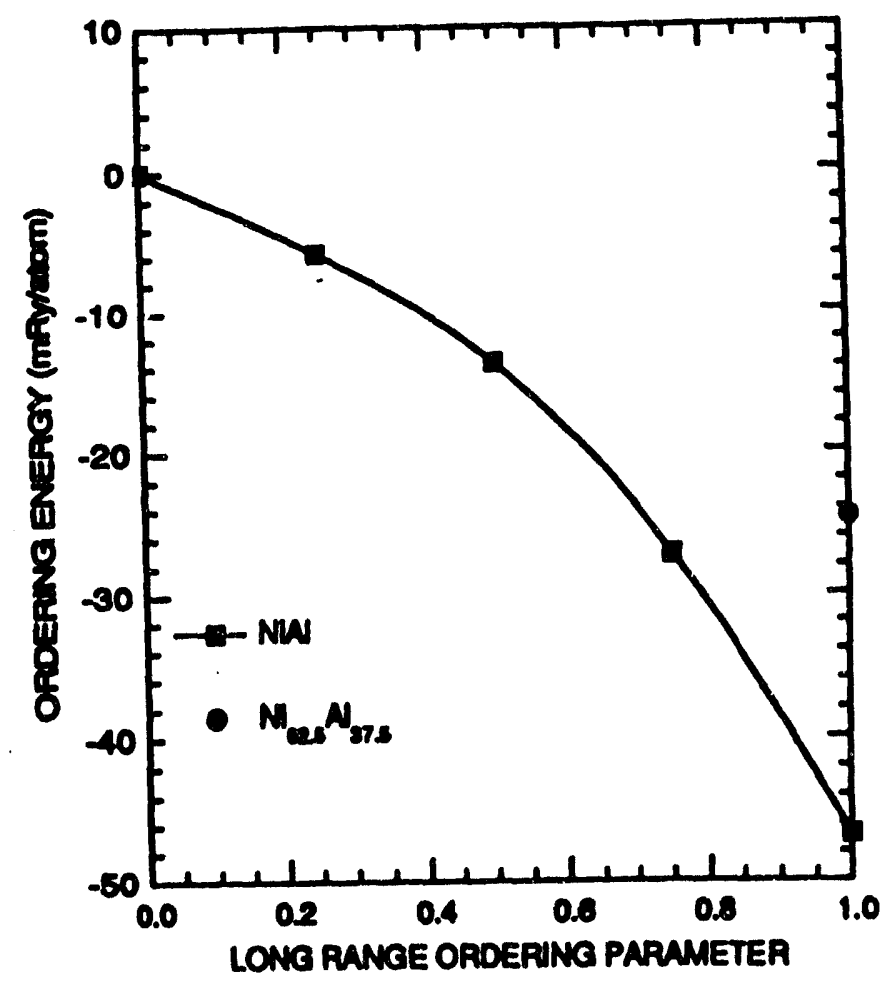

Figure 29: Ordering energy of $\mathrm{B2} \mathrm{NiAl}$ alloys as a function of the long range order parameter. The ordering eriergy for $\beta$-phase $\mathrm{Ni}_{0.0 .025} \mathrm{Al}_{0.375}$ is show by a solid dot 
The reason for this may lie in the effects of charge transfer since the degree of charge transfer between the Ni-sublattice and Atsublattice depends approximately linearly on $\eta$. Fig. 29 also shows (solid dot) the energy of ordered $\beta$-phase $\mathrm{Ni}_{0.625} \mathrm{Al}_{0.378}$ plotted with respect to the energy of the corresponding disordered bec phase.

In fig. 30 we show the densitjes of states for various values of the LRO parameter. Because of the large differences in the scattering cross-sections of $\mathrm{Ni}$ and $\mathrm{Al}$ sites

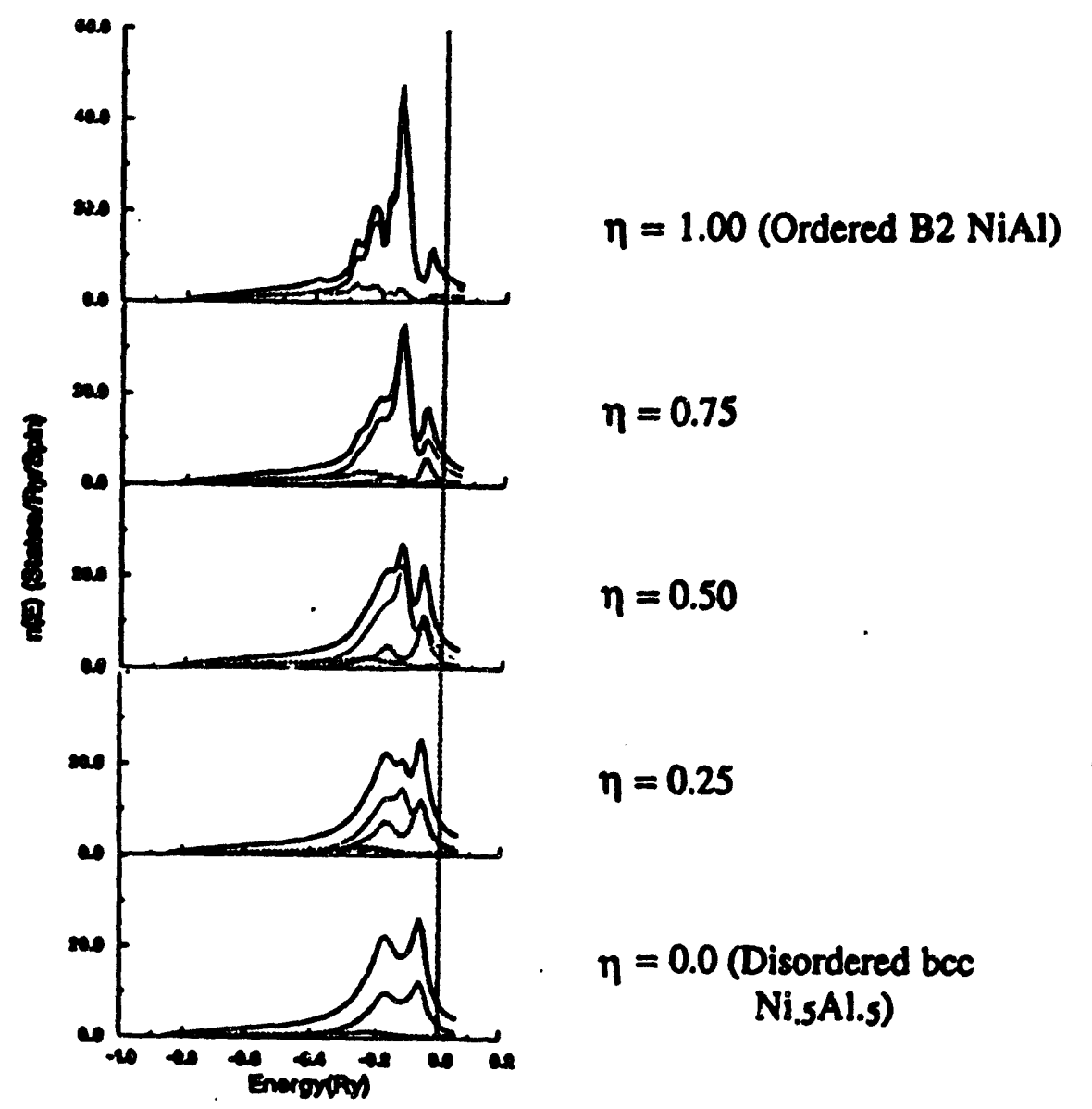

Figure 30: Densities of states of $\mathrm{B2} \mathrm{NiAl}$ alloys as a function of the long range order parameter. The total densities of states are given by the solid line. The heavy dotted lines give the densities of states on the $\mathrm{Ni}$-sub-lattice and the light dotted lines give the densities of states on the $\mathrm{Al}$-sub-lattice. In each curve the larger contribution arise from $\mathrm{Ni}$.

throughout the whole range of band energies, the DOS curve for the disordered alloy is relatively structureless. Clearly, upon ordering the weight in the DOS close to $\epsilon_{F}$ is reduced and the weight at low energies is increased resulting in stabilization of the B2 structure.

The changes in the densities of states showin fig. 30 result in a significant rearrangement of charge. In fig. 31 we show how the charge within equal volume Voronoi polyhedra associated with each sublattice evolve as a function of LRO. In the disordered state $\sim 0.3$ electrons/atom are transferred from $\mathrm{Al}$ to $\mathrm{Ni}$ sites. As the LRO builds up the atoms on their correct sub-lattice transfer even more charge whilst antisite atoms become more neutral. The net effect is to increase the Madelung energy over and above that associated with simple ordering of the charges associated with the $\mathrm{Ni}$ and $\mathrm{Al}$ sites in the disordered alloy. This effect is also stabilizing.

Before closing this section, we note that the order-disorder energy obtained from this direct calculation, $\Delta E^{\circ-d}(B 2, b c c)=46.7 \mathrm{mRy} /$ atom, is larger by a factor of two 


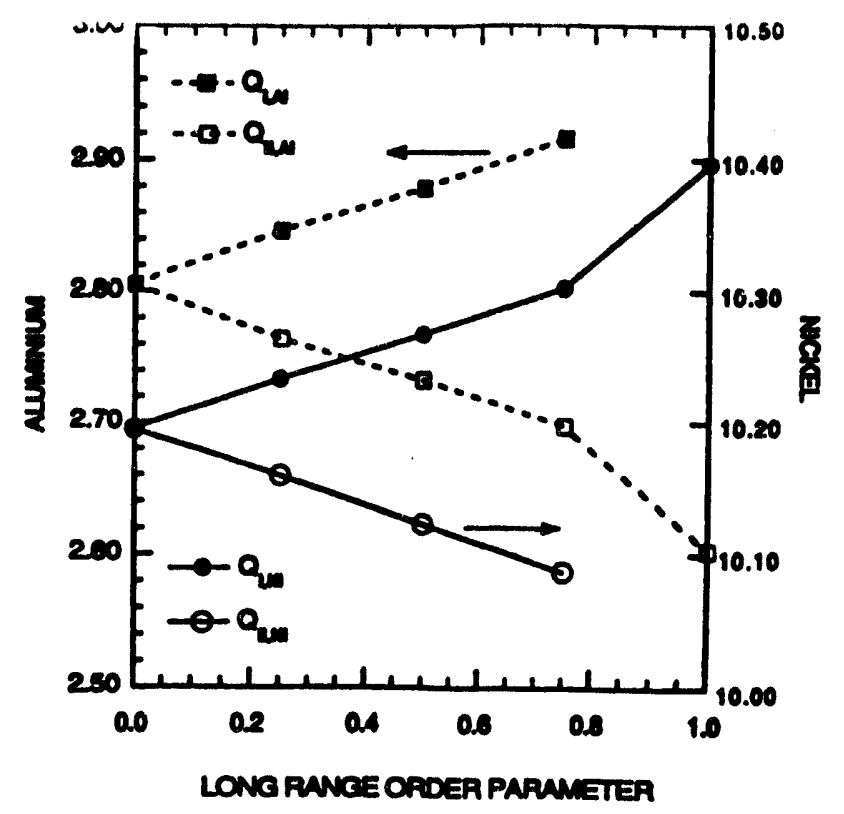

Figure 31: Evolution as a function of the LRO parameter of the charges inside equal volume Voronoi polyhedra associated with the $\mathrm{N} i$-sublattice (denoted by roman 1 ) and the $A$-sublattice (denoted by roman 2). Open squares(circles) give the charges associated with $\mathrm{Al}(\mathrm{Ni})$-atoms on the $A l$-sublattice. Solid squares(circles) give the charges associated with $\mathrm{Al}(\mathrm{Ni})$-atoms on the $\mathrm{Ni}$-sublattice.

than that obtained from the GPM interactions. If we consider only pairwise interactions out to third neighbor, the ordering energy for B2 is given by [102]

$$
\Delta E^{o-d}(B 2, b c c) \approx-V_{1}+\frac{3}{4} V_{2}+\frac{3}{2} V_{3} .
$$

Using the values of section 3.3 yields $\Delta E^{\circ-d}(B 2, b c c)=20.3 \mathrm{mRy} /$ atom. Clearly if the GPM interaction and disordered state energies are internally consistent the two methods should yield the same order/disorder energy. However, the GPM interactions were calculated on the basis of the band structure contribution $E_{B S}^{a}$ to the total energy alone, neglecting double counting corrections (contribitions that were included in the energy calculations), thus it is not necessarily surprising that the results differ. That the two values differ so much is presumably due to the relatively large charge transfer

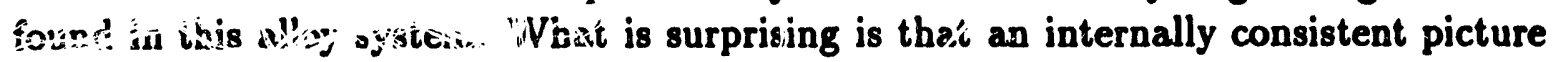
emeiges frors: the GPM $v$ is: ho charge transfer terms are neglected and they are used in conjunistion with the $\mathrm{K} K \mathrm{~K}$, A disordered state energy defined above. A resolution ai this dilemma may lay ds: a charge self-consistency scheme that goes beyond ine simple mean field theor; , so far. Work is in progress on this matter [36], [103]. This in turn may require : inarge transfer effects are also included in the GPM in order to regain a good descivition of the phase stability.

\subsection{Pre-Martensitic Phenomena in $\beta$-phase NiAl alloys}

Away from ideal stoichiometry $\beta$-phase $\mathrm{Ni}_{c} \mathrm{Al}_{1-c}$ alloys having compositions in the range $0.60<c<0.68$ transform Martensitically from the bcc-based B2 structure into fcc-derived structures. These alloys are interesting both from the technological point of view, in that for certain compositions they are candidate shape memory alloys, and from the point of lending themselves to fundamental studies of Martensitic phase transformations. 


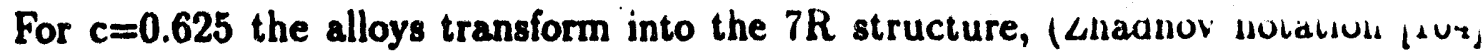
[105]), lower $\mathrm{Ni}$-content alloys transform into the $3 \mathrm{R}$ or $\mathrm{Ll} / 0$ structure. The Martensitic transition temperature $T_{M}$ is strongly concentration dependent varying from near $0^{\circ} \mathrm{K}$ at low Ni-content to well above room temperature for high Ni-content [106], [107]. Above $\mathrm{T}_{M}$ a number of pre-Martensitic phenomena are observed. Chief amongst thes: are "tweed" observed in transmission electron microscopy, the associated "streaking" observed in selected area electron diffraction [107], and the phonon anomalies observed in neutron scattering experiments [108], [109].

In fig. 32 we reproduce the inelastic neutron scattering experiments of Shapiro et al. [108], [109], [107]. .
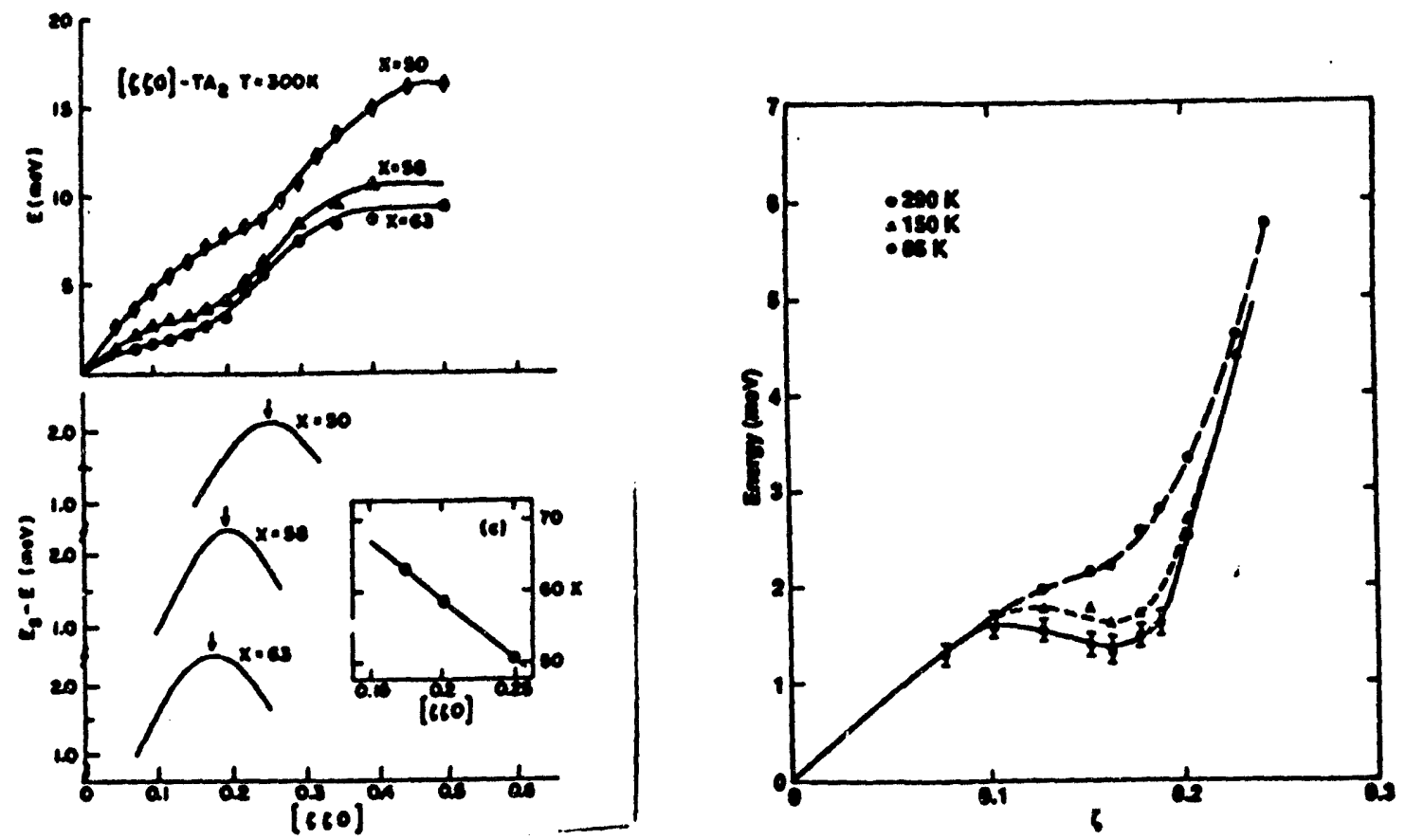

Figure 32: Concentration variation of the $\Sigma_{4}-T_{2}$ phonon branch (upper left) and of position of the dip in this branch (lower left and inset) in $\beta$-phase $\mathrm{Ni}_{x} \mathrm{Al}_{1-s)}$ alloys. Temperature dependence of the dip in the $\Sigma_{4}-\mathrm{TA}_{2}$ branch in $\mathrm{Ni}_{0.625} \mathrm{Al}_{0.375}$ (right) [108],[109], [107]

These plots revealed a concentration and temperature dependent dip in the $\langle\varsigma 50\rangle$ transverse acoustic branch of the phonon spectrum. At $c=0.50$, the dip is very shallow and is at a distance $g_{0} \approx 0.12(2 \pi / a)$ along the $\langle\varsigma s 0\rangle$ direction. The dip moves to larger values of $q_{0}$ with increasing concentration. At $c=0.625$ the dip is at $q_{0} \approx 0.16(2 \pi / a)$. For $\mathrm{Ni}_{0.625} \mathrm{Al}_{0.375}$ the dip becomes more pronounced as the temperature is lowered towards $\mathrm{T}_{M}$. Since the phase transformation is first order, the phonon frequency does not go to zero at $\mathrm{T}_{M} \approx 80 \mathrm{~K}$ as it would in an ideal soft phonon transformation. However, it is clear that this anomaly is closely connected with the phase transformation. Indeed, for $\mathrm{Ni}_{0.625} \mathrm{Al}_{0.375}$ the reciprocal of $q_{0}$ is close to the wavelength of the Martensitic 7R structure into which it transforms. Thus, understanding the mechanism that gives rise to the pre-Martensitic phonon softening can be expected to provide clues to the mechanism that drives the Martensitic transformation.

Recentiy, Zhao and Harmon [100] have, performed a detailed study of the phonon spectra of Ni-rich B2-phase $\mathrm{NiAl}$ alloys. These calculations are based on first principles calculations of the electronic structure of $\mathrm{B} 2 \mathrm{NiAl}$ which are then fitted to a nonorthogonal Slater-Koster tight binding Hamiltonian. This model Hamiltonian is then used to evaluate the electron phonon matrix elements using the method of Varma and 
Weber [110], [111]. In fig 33 we reproduce the calculated pnonon speuuda u. ......
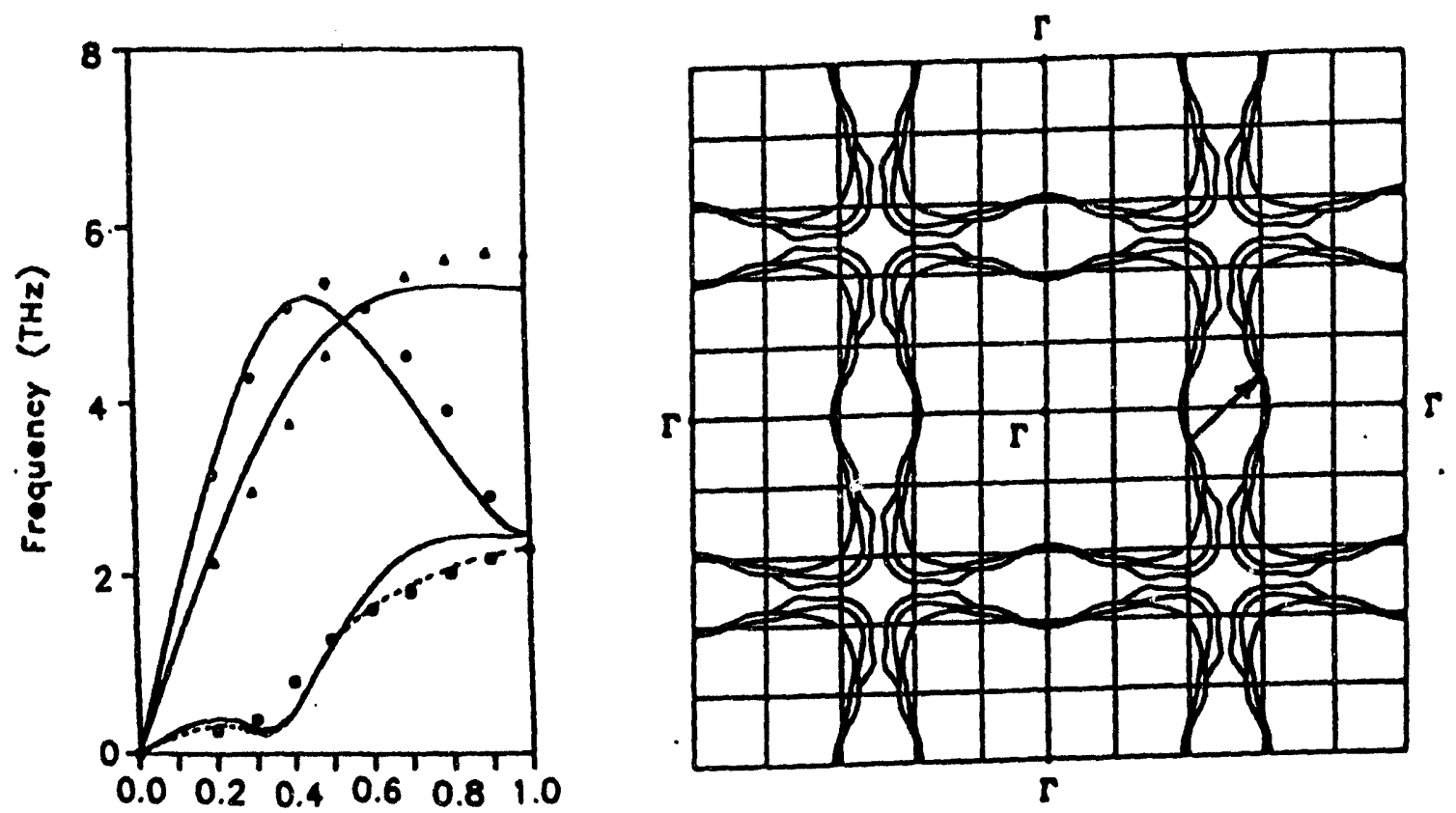

\section{$K / K \operatorname{mox} \quad[110\}$}

Figure 33: Left frame: Phonon dispersion curves of $\beta$-phase $\mathrm{Ni}_{0.625} \mathrm{Al}_{\mathbf{0 . 3 7 5}}$ for which a temperature of $1000 \mathrm{~K}$ has been used in the Fermi distribution function in order to simulate the effects of disorder in smearing the Fermi surface. Right frame: the Fermi surface showing the nesting feature. The $k_{x}-k_{y}$ with three different values of $k_{z}(0.25$, 0.38 , and $0.48 \pi / a$ are shown, and the arrow indicates two nested surfaces. After Zhao and Harmon [100]

and Harmon together with the Fermi surface that they obtained. Zhao and Harmon identified a Fermi surface nesting vector (marked by the arrow) that was responsible for the dip that they obtained in there calculations.

For pure metals and for ordered systems the methodology followed by Zhao and Harmon is quite sound since it is based on standard band theory technology. Unfortunately, for disordered alloys and off-stoichiometric compounds it is much less well founded. In order to be able to treat the off-stoichiometric $\mathrm{Ni}_{0.625} \mathrm{Al}_{0.376}$ compound, Zhao and Harmon made use of the rigid band model. They based their calculations on the $e^{\cdot}$ ectronic structure of stoichiometric NiAl assuming that the only effect on the electronic structure of adding excess $\mathrm{Ni}$ is to change the position of the Fermi energy. They choose valences of 1 and 3 for $\mathrm{Ni}$ and $\mathrm{Al}$ respectively (arguing that, in $\mathrm{Ni}$, the other nine $d$-electrons that are normally considered valence electrons are in a fixed $d^{\theta}$ core-like configuration), modify $\epsilon_{F}$ accordingly, and use this modified ordered compound band structure to perform the calculation of the phonon spectra. The problem with this approach is that the effect of off-stoichiometry, i.e. the creation of anti-site defects, is not only to modify the position of $\epsilon_{F}$, but also to smear out the electronic structure in a complex energy and $\vec{k}$ dependent manner. Thus the whole question of the existence of a band structure and Fermi surface is thrown into question.

As we have pointed out earlier a much better way to calculate the electronic structure of off-stoichiometric compounds is, at the outset, to face up to the fact that they are disordered and to use the LDA-KKR-CPA method. At the present time, there is no first principles theory of the dynamical matrix for disordered alloys similar to that of Varma and Weber. However, since the mechanism identified by Zhao and Harmon 
rests critically upon the picture of the electronic structure and fermı suriace obtasned using the rigid band model, we can inquire into its validity. Specifically, if the effect of adding disorder into the problem is to smear out those parts of the Fermi surface responsible for the nesting, this would negate the mechanism identified by Zhao and Harmon.

We have calculated the electronic structure of $\mathrm{Ni}_{0.628} \mathrm{Al}_{0.375}$ using the complex lattice LDA-KKR-CPA method. We assume that the structure is B2 and that the excess $\mathrm{Ni}$ goes onto the Al-sublattice. Thus the system is describe by two interpenetrating simple cubic lattices, one is occupied entirely by $\mathrm{Ni}$, i.e. has the composition $\mathrm{Ni}_{1.0} \mathrm{Al}_{0.0}$, the second has $\mathrm{Ni}$ and $\mathrm{Al}$ randomly distributed with composition $\mathrm{Ni}_{0.25} \mathrm{Al}_{0.73}$. Once the self-consistent crystal potentials have been obtained the $A^{B}(\vec{k}, \epsilon)$ was calculated using a generaliz: on of eq. 23. $A^{B}(\vec{k}, \epsilon)$ along a number of directions in the $\vec{k}_{x}, \vec{k}_{y}$ plane for a number of values of $\vec{k}_{z}$ is displayed down the left hand side of fig. 34 . For $\overrightarrow{\mathbf{k}}_{z}=0, A^{B}(\overrightarrow{\mathbf{k}}, \epsilon)$ is quite sharp in all directions, although, it is sharpeat along the $\Gamma-X$ direction and less sharp along $\Gamma-M . A s \vec{k}_{2}$ is increased $A^{B}(\vec{k}, \epsilon)$ remains sharp along $\langle 100\rangle$-directions for $\vec{k}_{z}<0.3(2 \pi / a)$ whilst along $(110\rangle$-directions it is rapidly smeared out by the disorder on the Al-sublattice. If we interpret the loci of the peaks in $A^{B}(\vec{k}, \epsilon)$ as the Fermi surface and the width as the inverse mean free path of the electrons, clearly the Fermi surface is well-define around the X-points in the Brillouin zone and much less so elsewhere.

On the right of fig. 34 we show a summary plot of the loci of the peaks in $A^{B}(\vec{k}, \epsilon)$. What is clear from this plot is that the Fermi surface is quite well-defined over a substantial region of the Brillouin zone around the $\langle 100\rangle$-direction. Since it is the Fermi surface in the neighborhood of this direction that, according to Zhao and Harmon, is responsible for the nesting, this is an important observation. Furthermore, in this region the Fermi surface is quite flat normal to the $\vec{k}_{2}=$ constant plane for values of $\vec{k}_{z}$ around $0.15(2 \pi / a)$.

In fig. 35 we plot the Fermi surface in the $\vec{k}_{z}=0.15$ plane in the extended zone scheme. We have also indicated the part of the Fermi surface that is welldefined and spanning vector that can give rise to nesting. The spanning vector is $\sim 0.18\langle 110\rangle(2 \pi / a)$, this is identical to that of Zhao and Harmon. Both are somewhat larger than the actual lock-in vector for the $7 R$ structure of $1 / 7(110)(2 \pi / a)$.

Even though we do not have a first principles theory of the phonon spectra in disordered off-stoichiometric compounds our results do lend strong support to the conclusions of Zhao and Harmon that the pre-Martensitic phonon softening observed in B2 phase $\mathrm{Ni}_{0.625} \mathrm{Al}_{0.375}$ is Fermi surface driven. Such an observation has important consequences for future theories in that is implies that the interactions giving rise to the pre-Martensitic behavior are long ranged. Thus we can conjecture that it will not be possible to describe this behavior on the basis of simple pair and short ranged interatomic potentials without the explicit inclusion of these subtle band structure effects. Since the effects of disorder on the electronic structure are extremely energy and $\vec{k}$ dependent, it is also clear that its effects will have to be considered at the outset. The extent to which such conclusions apply to the Martensitic phase transformation itself remains to be seen. That disorder is importan: in understanding the nature of the atomic displacements that precede the Martensitic transformation has already been provided by molecular dynamics studies based on embedded atom potentials [112]. In this work it was demonstrated that the characteristic "tweed" diffraction pattern is obtained for random positioning of the $\mathrm{Ni}$ and $\mathrm{Al}$ atoms on the disordered sublattice but it is not if the NiAl atoms are placed in some ordered array. Thus, it appears that a theory that includes both compositional and displacement fluctuations will be 

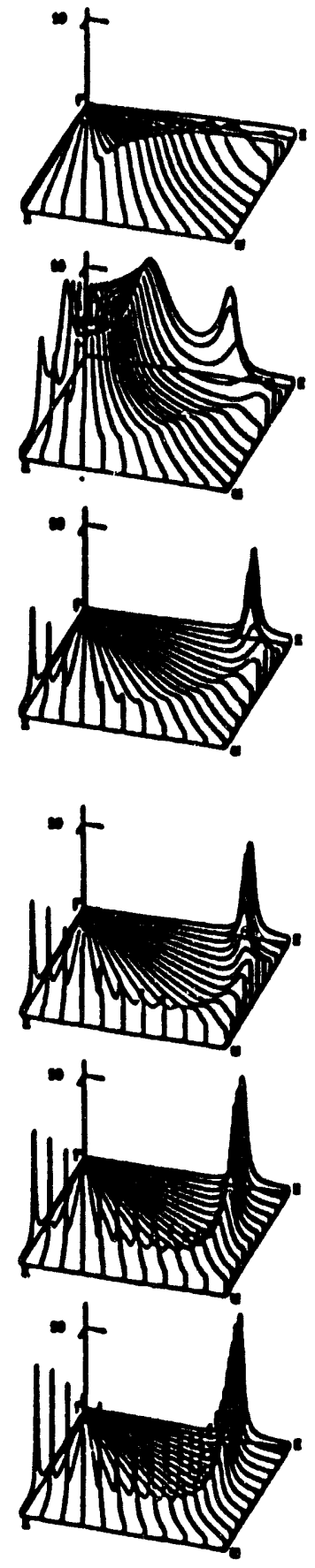

Fermi Energy Bloch

Spectral Functions

$z=.50$

for Ni62.5Al37.5
.40

- Simple cubic Brillouin zone in planes defined by $2=$ constant

.30

20

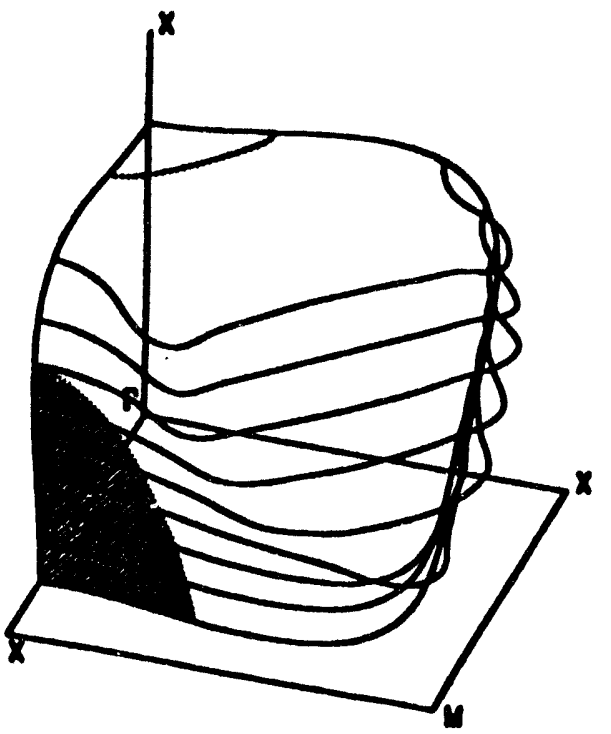

- Locus of peaks in BSF in various $\mathrm{z}=$ constant planes

$$
z=0
$$

Figure 34: Left: $A^{B}(\overrightarrow{\mathbf{k}}, \epsilon)$ along various directions in $\vec{k}_{2}=$ constant planes in one octant of the simple cubic Brillouin zone for off-stoichiometric $\mathrm{Ni}_{0.625} \mathrm{Al}$. 0.378 . Right: The loci of the peaks in $A^{B}(\vec{k}, \epsilon)$ in various $\vec{k}_{8}=$ constant planes. Starting from the basal plane the curves are for increments in $\vec{k}_{2}$ of 0.05 , i.e. twice the density shown on the left. The two uppermost curves are both for $\vec{k}_{z}=0.4$ since $A^{B}(\vec{k}, c)$ has two peaks. For values of $\vec{k}_{z}>0.4$ the peaks are so ill-defined that we did not plot their loci. The shaded area around the $X$-point indicates the region over which the Fermi surface is well-defined. 


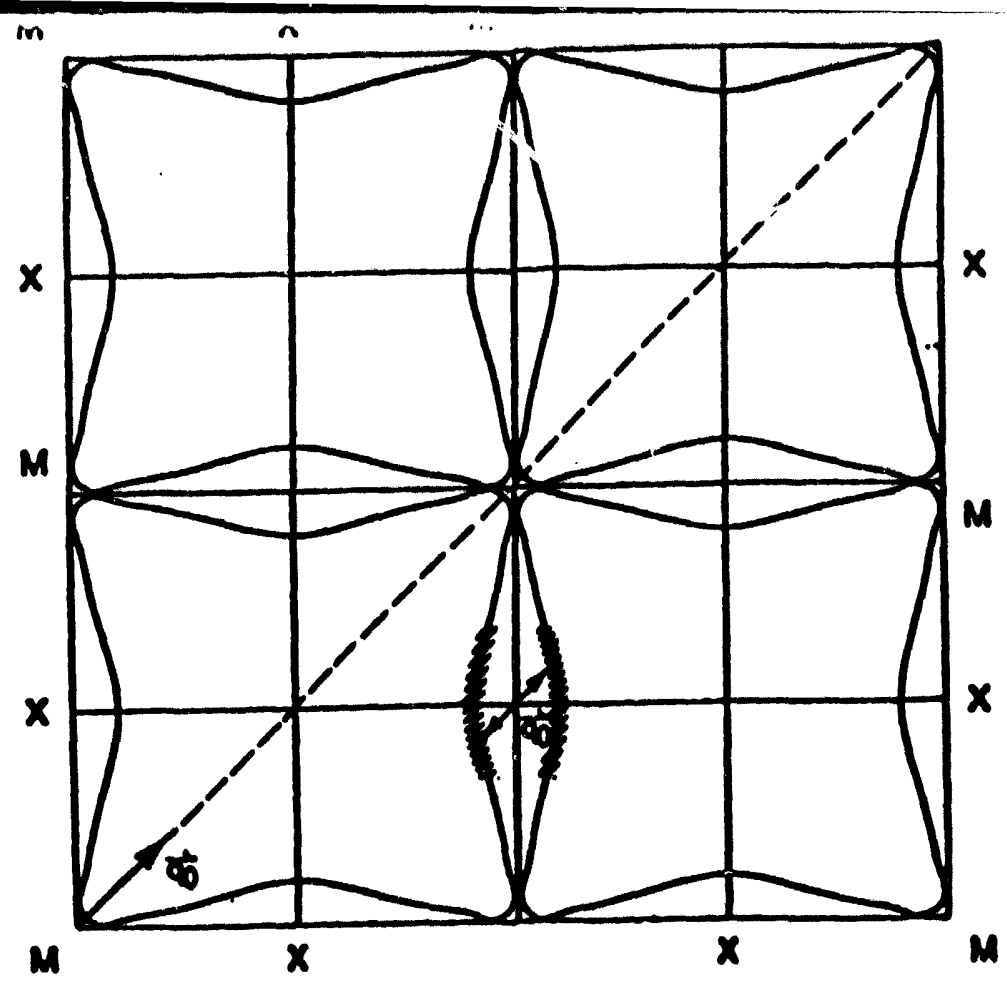

Figure 35: Fermi surface of off-stoichiometric B2-structure $\mathrm{Ni}_{0.625} \mathrm{Al}_{0.375}$ in the $\overrightarrow{\mathrm{k}}_{\mathrm{z}}=$ 0.15 plane over four adjacent Brillouin zones. The shaded region indicate where the Fermi surface is well-defined. We have marked by $\leftrightarrow$ a possible nesting vector.

required in order to fully understand these complex phenomena.

\section{Conclusions}

We have reviewed the local density approximation, first principles Korringa, Kohn, Rostoker coherent potential approximation theory of the electronic structure and energetics of substitutionally disordered alloys. We have also reviewed the mean field concentration functional and generalized perturbation method theories of ordering and alloy phase stability that have been built on the LDA-KKR-CPA description of the disordered state. Results of calculations of short range order parameters and phase diagrams for a number of alloy systerns have been presented that illustrate different ordering and clustering behavior. We have placed emphasis on understanding the driving mechanisms of ordering phenomena in terms of the underlying electronic structure. A number of mechanisms have been identified (Fermi surface nesting, band filling, offdiagonal randomness, relativity, charge transfer, magnetism, strain fuctuations, etc.) that provide a modern, first principles, basis for understanding ordering/clustering and alloy phase stability and for the interpretation of the Hume-Rothery rules and ideas based on tight-binding models. An important ingredient in this understanding is that features in the electronic structure of the disordered state thiat drive the ordering processes are also amenable to verification by experimental probes of the electronic structure of the disordered state. Thus, for example, the topology and smearing of an alloys Fermi surface that, on the one hand, determines its residual resistivity, can be responsible for the development of LPOS on the other.

To date, we have not made any numerical applications of the strain fluctuation theory presented in these notes. With the development of the anomalous X-ray scattering 


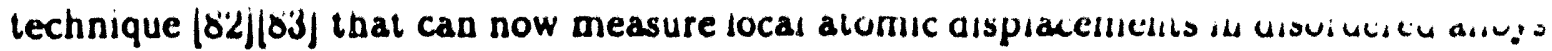
to a high degree of precision this promises to be a fruitful area. This is nowhere more likely than in the INVAR problem where concentration. magnetic, and displacement fuctuations are intricately coupled and in $\beta$-phase NiAl where concentration and displacement fluctuations appear as precureors to the Martensitic phase transformation.

Faulkner et. al. [113] have developed a new direct Monte Carlo approach that is based on the calculation of the energetics of specific local configurations embedded in an otherwise homogeneously disordered solid solution. The cluster energies are obtained from the embedded cluster method [19] which, in turn, is based on the LDA-KKRCPA treatment of the disordered phase. In many respecis the new method can be viewed as a next step beyond the MF-CF theory, in that, there is no need to partitinn the electronic energy between siteo, however, by going directly to the Monte Carlo method the statistical mechanics is treated essentially exactly, rather than the point approximation of MF-CF theory. Although only in the early stages of development the results obtained are very encouraging.

\section{Acknowledgments}

GMS: Work supported by Division of Materials Science Office of Basic Energy Sciences, US. Department of Energy under subcontract DEAC05-840R21400 with MartinMarjetta Energy Systems, Inc., and by a grant of computer time at NERSC from DOE-

BES-DMS. DMCN: Work supported by US Deparunent of Energy Assistant Secretary for Energy Efficiency and Renewable Energy, Office of Industrial Technologies, Advanced Industrial Materials Program, unde. subcontract DE-AC05-84OR21400 with Martin Marietta Energy Systems, Inc. FJP: Work partially supported by Cray Research, Inc., and the Ohio Supercomputer Center. BG: Work supported by Consiglio Nazionale Delle Ricerche (Italy).

\section{References}

(1) W. Hume-Rothery and G. V. Raynor, The Structure of Metals and Alloys, volume 3 of Mongraph and Report Series, The Institute of Physics, London, 1954.

[2] A. R. Miedema, P. F. de Chatel, and F. R. de Boer, Physica 100, 1 (1980).

[3] D. G. Pettifor, in Alloy Phase Stability, edited by G. Stocks and A. Gonis, volume 329, page 515, Boston, 1989, NATO-ASI, Kluwer.

[4] P. Hohenberg and W. Kohn, Phys. Rev 136, B864 (1964).

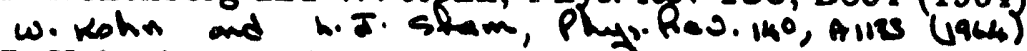

[5] P. Hohenterg and W. Kohn, Phyo.Rev 130, B864 (1064). - w

[6] S. Lundqvist and N. H. March, editors, Theory of the Inhomogeneous Electron Gas, Plenum, New York, 1983.

[7] P. Soven, Phys. Rev. 156, 809 (1967).

[8] D. W. Taylor, Phys. Rev. 156, 1017 (1967).

[9] B. L. Gyorffy, Phys. Rev. B 5, 2382 (1972).

[10] G. M. Stocks, W. M. Temmerman, and B. L. Gyorffy, Phys. Rev. Letters 41, 339 (1978). 
(11) D. D. Johnson, D. M. Nicholeon, F. J. Pinski, B. L. Lyortiy, and L. M. Slocks, Phys. Rev. Lett. 86, 2088 (1986).

[12] D. D. Johneon, D. M. Nicholeon, F. J. Pinski, G. B. L., and G. M. Stocks, Phys. Rev. 41, 9701 (1990).

[13] K. Binder, editor, Monte Carlo Methods in Statistical Physics, volume 7, Springer-Verlag, Berlin, 1979.

[14] K. Binder, editor, Applications of the Monte Carlo Method in Statistical Physics, volume 36, Springer-Verlag, Berlin, 1987.

[15] F. Ducastelle, Order and Phase Stability in Alloys, North Holland, Amsterdam, 1991, and references therein.

[16] J. W. D. Connolly and A. R. Williams, Phys. Rev. B27, 5169 (1983).

[17] Z. W. Lu, S. H. Wei, and A. Zunger, Phys. Rev. Letters 66, 1753 (1991).

[18] F. Ducastelle and F. Gautier, J. Phys. F 6, 2039 (1976).

[19] A. Gonis et al., Phys. Rev. B 36, 4630 (1987).

[20] B. L. Gyorffy and G. M. Stocks, Phys. Rev. Letters 50, 374 (1983).

[21] N. D. Mermin, Phys. Rev 137, A1441 (1965).

[22] L. Hedin and B. I. Lundqvist, J. Phys. C 4, 2064 (1971).

[23] P. Phariseau and W. M. Temmerman, editors, The Electronic Structure of Complex Systems, Plenum, New York, 1984.

[24] J. S. Faulkner and G. M. Stocks, Phys. Rev. B 21, 3222 (1980).

[25] B. L. Gyorffy and M. J. Stott, in Band Structure Spectroscopy of Metals and Alloys, edited by D. J. Fabian and L. M. Watson, New York, NY 10003, 1972, Academic Press.

[26] H. Winter and G. M. Stocks, Phys. Rev. B27, 882 (1983).

[27] J. F. Janak, Phys. Rev. B 8, 3985 (1974).

[28] H. Winter, P. J. Durham, W. M. Temmerman, and G. M. Stocks, Phys. Rev. B 33, 2370 (1986).

[29] G. M. Stocks and W. H. Butler, Phys. Rev. Letters 48, 55 (1982).

[30] W. H. Butler, Phys. Rev. 31, 3260 (1985).

[31] J. C. Swihart, W. H. Butler, G. M. Stocks, D. M. Nicholson, and R. C. Ward, Phys. Rev. Letters 57, 1181 (1986).

[32] P. E. A. Turchi et al., Phys. Rev. Letters 67, 1779 (1991).

[33] J. Murray, L. H. Bennett, and H. Baker, editors, Binary Alloy Phase Diagrams, volume 2, American Society for Metals, Metals Park, Ohio 44073, 1986. 
[34] R. Hultgren, P. D. Desai, D. Hawkins, M. Gleiser, and K. K. helly, S Values of the Thermodynamic Properties of Binary Alloys, Amercic for Metals, Metals Park, OH 44073, 1973.

[35] N. Saunders and A. P. Miodownik, in Alloy Phase Diagrams, edited by Bennett, B. C. Giessen, and T. B. Massalski, page 33, Pittsburgh, 1984, Mate Research Society, Materials Research Society, Extended Abstracts.

[36] D. D. Johnson and F. J. Pinski, unpublished.

[37] G. M. Stocks, F. J. Pinski, and D. D. Johnson, unpublished.

[38] W. B. Pearson, A handbook of Lattice Spacings and Structures of Metals and Alloys, volume 4, Pergamon Preas, New York, 1958.

[39] R. Evans, Adv. Phys. 28, 143 (1979).

[40] B. L. Gyorffy, D. D. Johnson, F. J. Pinski, D. M. Nicholson, and G. M. Stocks, in Alloy Phase Stability, edited by G. Stocks and A. Gonis, volume 163, page 421, Boston, 1989, NATO-ASI, Kluwer.

[41] B. L. Gyorffy et al., in Alloy Phase Stability and Design, edited by G. M. Stocks, D. P. Pope, and A. F. Giamei, volume 186, page 3, Pittsburgh, 1991, Materials Research Society.

[42] J. B. Staunton, B. L. Gyorffy, D. D. Johnson, F. J. Pinski, and G. M. Stocks, in Alloy Phase Stability, edited by G. Stocks and A. Gonis, volume 163, page 469, Boston, 1989, NATO-ASI, Kluwer.

[43] M. E. Fisher, J. Math. Phys. , 944 (1964).

[44] M. A. Krivoglaz, Theory of X-ray and Thermal Neutron Scattering by Real Crystals, Plenum, New York, 1969.

[45] L. Onsager, J. Am Chem. Soc. 58, 1468 (1936).

[46] R. Brout and H. Thomas, Physics 3, 317 (1967).

[47] J. Staunton and B. Gyorffy, Phys. Rev. Lett. 69, 371 (1992).

[48] D. Johnson, J. Staunton, and F. Pinski, to be published, presented at 1992 March Meeting, American Physical Society.

[49] D. M. Nicholson, G. M. Stocks, A. Gonis, and B. L. Gyorfy, unpublished.

[50] H. Sato and R. S. Toth, Alloying Behaviour and Effects in Concentrated Solid Solutions, Gordon and Breacl. New York, 1965.

[51] G. Ceder et al., in Alloy Phase Stability and Design, edited by G. M. Stocks, D. P. Pope, and A. F. Giamei, volume 186, page 65, Pittsburgh, 1991, Materials Research Society.

[52] M. E. Fisher and W. Selke, Phys. Rev Lett. 44, 1502 (1980).

[53] J. B. Cohen, in Solid State Physics, edited by H. Ehrenreich, D. Turnbull, and F. Seitz, volume 39, page 131, New York, 1986, Academic Press.

[54] A. Guinier, Nature(London) 142, 569 (1938). 
(55) G. D. Preston, Pror. R. Soc. London Ser. A 167, 526 (1938).

[56] A. Guinier, J. Phys. Radium (Paris) 8, 124 (1942).

[57] M. Cyrot and F. Cyrot-Lackmann, J. Phys. F 6, 2257 (1976).

[58] D. G. Pettifor, Phys. Rev. Letters 42, 846 (1979).

[59] J. V. der Rest, F. Gautier, and F. Brauers, J. Phys. F 5, 2283 (1975).

[60] V. Heine and J. Samson, J. Phys. F 13, 2155 (1983).

[61] A. Bieber and F. Gautier, Acta Metallurgica 34, 2291 (1986).

[62] V. L. Moruzzi, J. F. Janak, and A. R. Willaims, Calculated Electronic Properties of Metals, Pergamon, New York, 1978.

[63] C. E. Dahmani, M. C. Cadeville, J. M. Sanchez, and J. Morán-Lopez, Phys. Rev. Letters 55, 1208 (1985).

[64] G. Treglia and F. Ducastelle, J. Phys. F 17, 1935 (1987).

[65] F. Pinski et al., Phys. Rev. Letters 66, 766 (1991).

[66] Z. W. Lu, S. H. Wei, and A. Zunger, Reciept of a preprint is gratefully acknowledged, 1991.

[67] Z. W. Lu, S. H. Wei, and A. Zunger, Phys. Rev. Lett. 68, 1961 (1992).

[68] F. J. Pinski et al., Phys. Rev. Lett. 68, 1962 (1992).

[69] B. L. Gyorffy et al., in Structural and Phase Stability of Alloys, edited by J. L. Morán-López, F. Mejía-Lira, and J. M. Sanchez, New York, 1992, Plenum Press.

[70] B. Ginatempo, G. A. Geist, G. M. Stocks, W. A. Shelton, and E. Bruno, in First Workshop on Vectorial and Parallel Computing of the Eleatronic Properties of Solids, edited by A. Stancanelli, E. S. Giuliano, and B. Ginatempo, volume Classe di Scienze Fisiche Matematiche E Naturali LXIX Supplemento 2, Messina, 1991, Accademia Peloritana Dei Pericolanti.

[71] D. D. Johnson, F. J. Pinski, and G. M. Stocks, J. Appl. Phys. 57, 3018 (1985).

[72] D. D. Johnson, F. J. Pinski, and J. B. Staunton, J. Appl. Phys. 61, 3715 (1987).

[73] D. D. Johnson, J. B. Staunton, F. J. Pinski, B. L. Gyorffy, and G. M. Stocks, in Phyisical Metallurgy of Controled Expansion INVAR-type Alloys, edited by K. C. Russell and D. Smith, The Metallurgical Society Society, The Metallurgical Society, 1989.

[74] D. D. Johnson, J. B. Staunton, F. J. Pinski, B. L. Gyorffy, and G. M. Stocks, in Applications of Multiple Scattering Theory to Materials Science, edited by W. H. Butler, P. H. Dederichs, A. Gonis, and R. L. Weaver, volume 253, page 277, Pittsurgh, 1992, Materials Research Society, Materials Research Society.

[75] J. B. Staunton, F. J. Pinski, and D. D. Johnson, Phys. Rev. Lett. 65, 1259 (1990).

[76] J. W. Cable and W. E. Brundage, J. Appl. Phys. 53 (1982). 
[77] J. Cable, H. R. Child, and Y. Nakai, Physica 150 and 156B, 50 (198y).

[78] A. G. Khachaturyan, Theory of Structural Transformations in Solids, Wiley, New York, 1983.

[79] A. Zunger, S. H. Wei, A. A. Mbaye, and L. G. Ferreira, Acta Metallurgica 36, 2239 (1988).

[80] J. M. Sanchez, Phys. Rev. B 45, 2015 (1992).

[81] N. Mousseau and M. F. Thorpe, Phys. Rev. B 45, 9999 (1992).

[82] G. E. Ice, C. J. Sparks, A. Habenschuss, and L. B. Shaffer, Phys. Rev. Lett. 68, 863 (1992).

[83] L. Reinhard et al., Phys. Rev. B 15, 2662 (1992).

[84] A. Zunger, S.-H. Wei, L. Ferreira, and J. Bernard, Phys. Rev. Letters 65, 353 (1990).

[85] S. Marias, V. Heine, C. Nex, and E. Salje, Phys. Rev. 66, 2480 (1991).

[86] F. Ducastelle, in Alloy Phase Stability, edited by G. Stocks and A. Gonis, volume 163, page 293, Boston, 1989, NATO-ASI, Kluwer.

[87] P. E. A. Turchi, G. M. Stocks, W. H. Butler, D. M. Nicholson, and A. Gonis, Phys. Rev. B 37, 5982 (1988).

[88] M. Sluiter and P. E. A. Turchi, in Alloy Phase Stability, edited by G. Stocks and A. Gonis, volume 521, page 515, Boston, 1989, NATO-ASI, Kluwer.

[89] M. Sluiter, P. E. A. Turchi, F. J. Pinski, and G. M. Stocks, Journal of Phase Equilibria 13, 605 (1992).

[90] P. E. A. Turchi, M. Sluiter, and G. M. Stocks, Journal of Phase Equilibria 13, 391 (1992).

[91] N. Saunders, A. P. Miodownik, and A. T. Dinsdale, Calphad 12, 351 (1988).

[92] P. E. A. Turchi, M. Sluiter, F. J. Pinski, and G. M. Stocks, page 59, Amercian Society for Metals, Amercian Society for Metal, 1992, to be published.

[93] A. Finell and F. Ducastelle, in Phase Transformations in Solids, edited by T. Tsakalakos, volume 21, page 59, Amsterdam, 1984, Materials Research Society, North Holland.

[94] D. de Fontaine, Solid State Physics 34, 73 (1979).

[95] N. Saunders, A. P. Miodownick, and A. T. Dinsdale, Calphad 12, 351 (1988).

[96] R. W. Cahn, in High Temperature Ordered Alloys 2, edited by N. Stoloff, C. C. Koch, C. T. Lui, and O. Izumi, volume 81, page 27, Pittsburg, 1987, Materials Research Society, Materials Research Society.

[97] A. Pindor, B. L. Gyorffy, and W. M. Temmerman, Joural of Physics F: Metal Physics 13, 1627 (1983). 

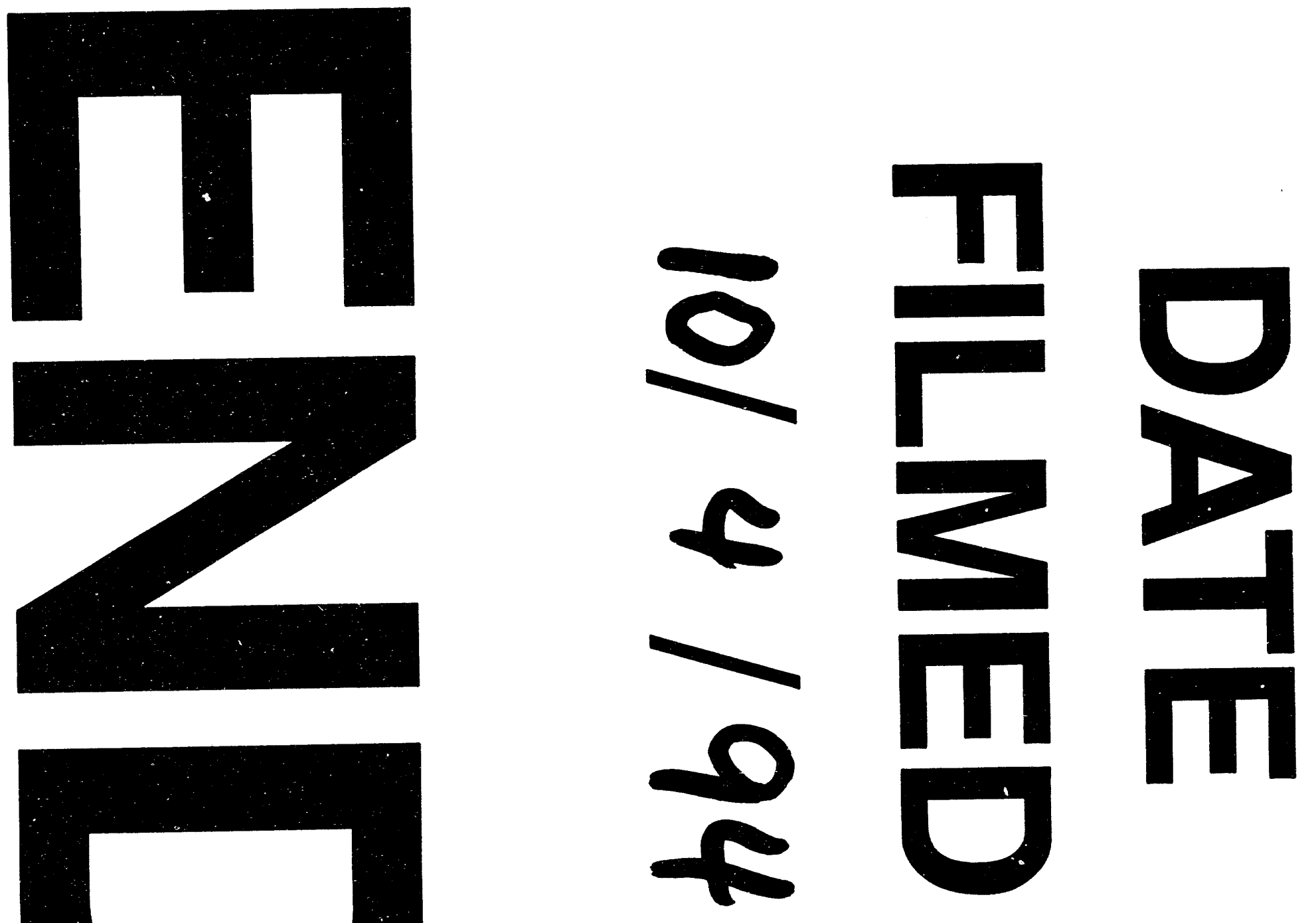
